\title{
A Quantum Cognitive Perspective on Control and Arousal
}

\author{
Lena Rosendahl, Jonathan Cohen *
}

March 2021

*funded by the Templeton Foundation 


\begin{abstract}
In this article, we consider a quantum approach to modeling the influence of control and arousal on decision making. This builds on the multi-particle multi-well (MPMW) framework, introduced in [47] and [48], that uses quantum potential landscapes to provide a mathematically tractable framework for analyzing multi-alternative decision making, and in which stochasticity emerges, alongside leak and competition, as an inherent property of the system rather than requiring treatment as a free parameter. Here, we show how the MPMW framework can be extended to model phenomena associated with cognitive control, such as the effect of control on processing conflict, and the stability-flexibility tradeoff. Furthermore, we show that the MPMW framework, by its treatment of arousal, can be extended naturally to provide a mathematically coherent account of a broader class of psychological phenomena, such as the non-monotonic relationship between arousal and performance. In addition to seamlessly accommodating both control and arousal, the the MPMW predicts some unusual effects, such as discontinuous changes in performance with changes in attention and arousal, that emerge as a result of its quantum foundations.
\end{abstract}

\title{
1 Introduction and Background
}

\subsection{The Drift Diffusion Model (DDM)}

Forced-choice decision making under uncertainty and time constraints is a common real-world problem faced by agents functioning in natural environments, including humans, non-human animals and artificial agents. As such, the mechanisms responsible for decision making processes have been studied and modeled extensively throughout decades of work in psychology, neuroscience, economics, and related disciplines ([10],[57],[50],[42]).

Two-alternative forced choice (2AFC) decision making provides a simplified decision-making paradigm that is tractable to formal analysis, and thus is the most commonly treated paradigm among decision-making models. Among many models of this paradigm, the continuous time drift diffusion model (DDM) [45] has arisen as the gold standard. In addition to replicating important properties of human behavior in 2AFC tasks (e.g., inverse Gaussian response time (RT) distributions, and effects of biases on decision mak- 
ing), the DDM implements an optimal procedure for such decision making in that it minimizes response times for a given level of accuracy or maximizes accuracy for a given response time. In addition, the DDM provides a means for conducting an optimality analysis of the speed-accuracy tradeoff inherent in 2AFC decision making by identifying the decision threshold that maximizes reward rate for a given set of task parameters (i.e., signal strength, noise, etc.). This provides a means for making quantitative predictions about the behavior of the system at optimal threshold ([6]). These properties allow normative modeling under the DDM, quantitatively predicting adjustments in parameters that optimize performance, in turn making testable predictions about behavior, many of which have been validated (see $[5],[2])$. However, there are several limitations of the DDM: it is difficult to extend to decisions involving more than two alternatives; it places no bound on the amount of information per unit time that a system may accumulate; and it treats stochastic variance (noise) as a free parameter of assumed form that must be estimated from empirical data.

\subsection{Classical and Quantum Extensions to the DDM}

A number of classical extensions and alternatives to the DDM have been implemented to address these issues, including frameworks for multi-alternative forced choice decision making [53],[19],[4], DDMs with variable drift rate, and alternatives such as decision field theory and the leaky competitive accumulator (LCA) model [57], [10]. However, each comes with its own limitations, such as restriction to extreme conditions (e.g., maximal accuracy), difficulty mapping to greater than two alternatives, lack of mathematical tractability when a model does treat multiple alternatives and, for all of them, the lack of a principled account of noise. Furthermore, while some of these approaches have been used to address the effects of cognitive control (see [13],[38]), to our knowledge none has provided a formal treatment of a psychologically ubiquitous phenomenon: arousal.

There are also a number of models that take a quantum approach to describing perceptual and preferential decision making (e.g., [10], [12], [9], [11], [32], and [48]). These have been shown to successfully reproduce many of the phenomena addressed by classical models, and predict new ones that have begun to receive empirical support ([28]). However, while noise arises in a principled way from these quantum approaches, they face some of the limitations noted above (e.g., they do not address multi-alternative decision 
making), and they have not been used to address the influence of attentional control on processing. Furthermore, they do not address the influence of energy in the system, a fundamental factor in quantum formulations, that we propose can be used to formalize the influence of arousal on decision making.

\subsection{Overview of the MPMW Framework}

Recent work introduced a new quantum approach to modeling decisionmaking, the Multi-particle Multi-well (MPMW) framework [48], that was designed to address these limitations. This was applied in its simplest form to the $2 \mathrm{AFC}$ problem, showing that the MPMW framework shares many of the properties of the DDM, including its formal optimizability, while making several new predictions. In this article, we extend that work to show how the MPMW can be used to address some of the important limitations of the DDM and other approaches to modeling decision making. Specifically, we show how it can be used to address multiple alternative decision making, reproduce effects associated with parameters such as leak and variance in classical models as principled emergent properties of the framework rather than as free parameters that must be explicitly specified, and provide a formal treatment of the effects of control and arousal on decision making.

To motivate this work, we consider how the MPMW differs from previous quantum and classical approaches. First, it treats the signal (stimulus) as a process that generates a sequence of quantum particles, each of which represents a single bit of information, rather than a single particle. Second, the particles are acted upon by a set of non-uniformly spaced and sized attractor wells (hereafter, "wells"), each corresponding to a distinct stimulusresponse pairing, rather than a lattice of uniformly spaced and sized wells all associated with one task parameter (as in [12]). The well parameters - depth, width, and separation - are determined by stimulus or task properties both internal and external to the agent's representational system, all of which we address shortly. The decision process involves making a measurement of each particle's position, collapsing it to a single point either within a well or the "classically forbidden region." The latter, that we discuss further on, is not a possibility in previous classical or quantum treatments. Finally, a decision is made when the total number of particles that have been measured in one well exceeds those in others by a specified number (the decision "threshold"; for a detailed treatment of the two-alternative case, see [48]), and the identity 
of that well determines the outcome of the decision.

Although the decision process in the MPMW framework involves integration, as in classical decision-making models and single particle quantum treatments, it differs in that quantum particles are initially distributed over all wells in the landscape and the forbidden region. The probability of measuring a particle's position to be within a particular well is labeled as the well's mean integration efficiency (MIE), a quantity defined by the well's parameters (depth and width) and its proximity to other wells. In other words, MPMW describes the integration process as the sum of a series of independently distributed variables. In its simplest form, these variables are also identically distributed (IIDs), as in classical models such as the DDM. However, the distribution of the variables is not assumed to be Gaussian. Rather, it is described by the MIEs, which are determined by a simple set of context-specific variables that we address in detail throughout this article. Furthermore, the discrete and bounded nature of MIEs differ from the continuous and unbounded nature of drift rate (and variance) in the DDM. This results in an additional component to non-decision time (ndt), not present in the DDM or other classical models, that imposes a lower boundary on the system's thresholds: Decisions cannot be made before a minimum time

$$
t=\frac{\min \left(T_{f}, T_{s}\right)}{m}
$$

where $T_{f}$ and $T_{s}$ are the decision thresholds associated with failure and success respectively, and $m$ is the number of integrators applied to the task. Evidence for discrete increases in evidence accumulation for individual neurons has been uncovered by recent work [29], [37], [20].

Finally, like other quantum models, but unlike classical ones, stochasticity arises directly from the interaction between the particle and landscape, rather than being explicitly specified by a noise parameter. This means that empirically observed variance can be used to determine the properties of the underlying quantum landscape. These conceptual differences lead to predictions of the dynamics of decision variables that differentiate their behavior from the DDM and other quantum models.

Rosendahl \& Cohen 2021 [48] considers some of the differences between the MPMW and other frameworks, focusing on its "classical component"; that is, the integration process under fixed landscapes and MIEs. Here, we extend this to address how landscape parameters determine the MIEs, producing "dropped bits" (analogous to "leak" in LCA models) and competi- 
tion (i.e., conflict between competing responses and/or tasks) that depend on both the landscape and the system energy (i.e., arousal). We show that using quantum potential landscapes to model internal representations of tasks and stimuli predicts discontinuities in performance curves as functions of stimulus, task properties, arousal, and interactions among these. Additionally, we show how, under the MPMW, the magnitude and variation of arousal play fundamental roles in determining how information is accumulated. In the sections that follow, we begin by providing a more detailed description of the motivation for and fundamental principles of the MPMW framework. We follow this with specific examples of how it can be used to model wellestablished phenomena concerning decision-making and control, such as the Stroop Effect C, as well as less-commonly modeled phenomena associated with arousal, for which MPMW makes novel predictions.

\section{Principles of the MPMW Framework}

\subsection{Representing Incident Stimuli by Quantum Parti- cles}

One of the distinguishing properties of the MPMW framework is its treatment of incident stimuli as a set of quantum particles rather than as a single one. There are two reasons for this choice. The first is that the arrival of information can be treated as occurring in discrete increments; that is, as a discrete time information accumulation process over one more more integrators. This places a bound on the amount of information received per time step per integrator. This differs subtly but critically from the classical sequential probability ratio test (SPRT), which treats the information integration process as a sum of the logs of likelihood ratios of observations. Despite the assumption of continuous time, work that fits the DDM to empirical data imposes discrete time upon the model, with the magnitude of the time step as a free parameter that must be determined for fitting.

Though the DDM has been used successfully to model a variety of empirical phenomena, both at the neural and behavioral levels, how this corresponds to discretization at either level of processing is an open question. Discretization of processing is observed both within the brain as quantal release of neurotransmitters (see [41], [21] \& [52]), the binary nature of action potentials, and in proposed mechanisms of gating at the population level 
(see [31], [36], and [55]) and at the cognitive level (e.g., the discretizing effects of selective attention on processing (see [62], [61], [60], [8]). Quantum approaches treat discretization as a fundamental property (quantal events that have intrinsic stochasticity) to which multivariate methods of analysis are applied. Not only may this provide a more natural approach to characterizing discrete phenomena observed at the neural and cognitive levels of processing, but it also may provide an account of variance in behavior as an emergent property of the system, rather than as a free parameter that must be fit to data.

The second motivating factor for the quantum approach is that, at each moment in time, an agent will perceive, at a low level, vastly more information than that to which it can meaningfully respond. Within the quantum framework, this can be treated as a single particle (packet or bit) containing the entirety of the information that is transmitted to the agent, but with its components represented in a way that is determined by the agent's processing biases. When that information is processed by an integrator (e.g., one or a pool of neurons), it is generally assumed that the integrator is sensitive to only a subset of the information present in the initial distribution [46]. As a consequence, when the integrator represents the stimulus, the stimulus is categorized as arising only from that subset. This process, in which a rich representation that contains a large amount of information about the environment is reduced to a representation that captures and integrates only a subset of that information, is analogous to one in which a quantum particle arriving from the environment in a distributed state (i.e., containing rich information about the environment) is collapsed, through measurement by a detector, to a state localized to the detector.

It is important to note that the application of tools from quantum mechanics does not imply that the brain is literally a quantum computer. Rather, it simply makes certain assumptions about representations, and then models information accumulation processes using tools from quantum theory. The appeal of the approach is that it may provide a more natural, and analytically tractable description of those processes, that accurately reproduces relevant empirical phenomena, including subtleties that are not easily addressed by purely classical models. This approach, in a more general form, has proven useful in the development of other quantum cognitive models applied to different areas of decision-making (see [12], [9], [11], [54], [28]). 


\subsection{The Information Integration Process}

The decision process model used in the MPMW treats each particle of incident information as arriving and being processed sequentially, each by an integrator. This is analogous to a particle (such as a single photon or muon) being emitted into a room, the back wall of which is arrayed with detectors. When it arrives at a detector, the detection process both measures its position (collapsing its distributed state to a single point) and transforms it to a different form of energy, effectively clearing the particle from the room. After the detection takes place, another particle is emitted and the process repeats. In this system, if each particle is emitted with the same energy, there is a fixed probability that the particles will be measured by each detector: the probability that the particle is found to occupy that detector's position. The array of may be represented by a one-dimensional attractor landscape, any point in which the particle may occupy at any given time after emission. In the MPMW, each position is treated as corresponding either to a task-relevant single decision alternative, or to neutral task-irrelevant alternatives (a detector that does not transmit its data) in a forced choice task. If a particle is measured to be in the region associated with a task-relevant alternative, then a single bit of evidence is accumulated in favor of that alternative, otherwise it is ignored. This formulation allows the decision variable to be reduced to a Markov variable. The dynamics for its progress in a $2 \mathrm{AFC}$ decision are described by [48].

\subsection{Four Critical Parameters of MPMW Models}

Well depth The simplest formulation of a quantum square attractor landscape is a single square well of infinite depth. Infinite depth allows the landscape to be treated simply as a set of boundaries, corresponding closely both to classical models of decision making, and to previous quantum approaches (e.g., [12]). However, it imposes the constraint that the particle cannot occupy any space outside the well, thus eliminating the possibility for lost information or changes in the integration process due to changes in arousal. Such a model implies infinite attentional capture, which is implausible and disallows changes in attention to non-target stimuli or changes in processing due to shifts in arousal. Furthermore, while this approach could be generalized to decisions with multiple alternatives by considering higher dimensional wells, doing so would abolish its appealing simplicity and would 
not allow for the treatment of differences in stimulus-response properties.

As noted above, the MPMW takes a different approach by assigning each stimulus-response association to a distinct well and using finite well depth to model the efficacy with which a given alternative is processed. Processing efficacy is assumed to be co-determined by the salience of a stimulus, its familiarity (i.e., the automaticity with which it can be processed), and the allocation of attention (whether induced through automatic capture or predetermined by allocation of control mechanisms). Assigning all factors associated with attentional capture to depth in this way aligns with the modeling of these factors in neural networks in terms of the common underlying mechanism of connection strength (e.g., [16]). In the remainder of this article, we use the term "efficacy" to refer collectively to these factors, and associate this with the parameter of well depth.

Well width and separation Because the MPMW framework assigns each stimulus-response pairing to a distinct well, it requires that the width of each well and its separation from others on the landscape also be considered. The MPMW associates these parameters with semantic properties of representations: well width with the abstractness or generality of a representation, which we term "representational generality", and separation (i.e., proximity) with the similarity among representations ("representational similarity"). Thus, more general concepts (e.g.,the dimension "color") are assumed to be represented by wider wells than more specific concepts (e.g., a particular color); and similar representations (such as "red" and "green") are assumed to be nearer to one another than more dissimilar ones (e.g., red and dog). The latter determines the extent to which one well influences the component of a particle's state that is localized to another well. Thus, the closer wells are to one another, the greater the likelihood of interference between them.

Energy Considering a landscape comprised of multiple wells that vary in depth, width and separation requires, in turn, that the energy of the system be specified. This, alongside the other parameters, determines the likelihood that the particle will be detected within any particular well (a well's MIE) or in the "classically forbidden region" between or beyond the wells. The MPMW maps the energy of the system to arousal. As we show below, this yields a performance curve qualitatively similar to that of the classic Yerkes- 
Dodson curve [64], with both extremely low levels and extremely high levels of arousal producing poor or absent performance, and mid levels of arousal associated with optimal performance, that has sometimes been referred to as "flow" [58]. However, in additional to providing a principled formulation of arousal, treating it as energy predicts that, unlike the Yerkes-Dodson curve, the relationship to performance is not symmetric or perfectly general. Rather, it is determined by context in ways that make novel and interesting predictions.

In summary, a full treatment of an MPMW model involves four critical parameters that determine the processing characteristics of the system: well depth, width, separation, and the energy of the system. In the remainder of this article, we consider how mapping these to cognitive factors can provide both an integrative and analytically tractable account of wide range of wellestablished empirical phenomena, as well as a number of novel predictions.

\section{Finding MIEs by Solving the Schrodinger Equation}

The formal foundation of the MPMW rests on the ability to mathematically determine the probability of detecting a particle within a particular well (the well's MIE) by solving the Schrodinger Equation associated with the attractor landscape. This is a Hamiltonian describing the distributed state and dynamics of a quantum particle by the sum of its kinetic energy and the potential acting upon it. The potential energy landscape, $V(x)$, describes the amount of attractive or repulsive force acting upon a particle in the region $x$, pulling it into or pushing it out of that region and its kinetic energy is simply a scalar function of its squared momentum. The potential energy landscape in an MPMW model represents the probability of a bit of information being integrated as evidence for each stimulus-response pairing, with larger and stronger (wider and deeper) attractors producing stronger internal representations.

This is similar, but not identical to a classical attractor landscape. The latter is often described as a set of hills and valleys (analogous to the potential energy landscape) in which a ball (particle) is rolling (the kinetic energy). Classically, a ball will either settle into a local valley or keep rolling out of the region depending on its starting point and initial kinetic energy. However, 
unlike a classic system, regardless of energy, a quantum particle will not settle into a single valley, but will occupy a state that is probabilistically distributed across all hills and valleys depending on its energy and the depth and width of each valley. This distributed state is the particle's position and directly produces the probability distribution of its measured position.

In section 3.2, we describe the form of the solutions of the Schrodinger Equation for models implemented in the MPMW framework. First, however, we introduce important assumptions the MPMW framework makes about the landscape, including the use of the Time-Independent Schrodinger Equation (TISE), and the meaning of bound states, that underlie the results we go on to describe.

\subsection{Important assumptions}

In this section we discuss several crucial technical assumptions that the MPMW framework makes about the underlying quantum processes that define the MIEs and, thereby, the Markov decision variable, and that are necessary for its analysis. Briefly, we assume that the potential landscape is piecewise constant, each alternative is assigned to a well of finite width and depth, and that it is appropriate to use the bound states of the TISE to represent the MIEs of a system. Readers who are not interested in the justifications of these assumptions may skip this section.

\subsubsection{The Piecewise Constant Landscape}

First, for simplicity of representation, where $N$ is the number of competing stimuli, the MPMW framework divides the one-dimensional landscape into $2 N+1$ regions. Each region is assigned a constant potential value, $V_{r}$. Each task-associated "well" has an independent potential value $V_{\max }-d_{k}$ (where $k$ is simply the stimulus index) and the "barrier" regions separating the wells from one another have a constant potential value $V_{\max }$. Taken together, the barrier regions are called the "classically forbidden region" for reasons that will become clear shortly. Increasing attractor strength can be thought of as increasing the depth of a well and, in general, contributes to a higher probability of a particle being measured in that region (i.e., a higher MIE for the region, or $M_{k}$ ). For a given well, increasing the region of attraction corresponds to increasing the width of the well, and maps to an increase in the generality with which a stimulus is represented (e.g., in classifying colors 
as either red or orange, how much yellow can be present before a color is less likely to be categorized as red than orange). On either side of a set of wells (regions 1 and $2 N+1$ ), the landscape is assigned a constant potential value $\left.V_{\max }\right)$ (corresponding to the top of all wells), and extends infinitely in either direction. Alternatively, a one-dimensional landscape may also be placed on a ring, in which case these regions are simply replaced by a single barrier region with the same value $V_{\max }$. The well widths are defined by a set of bounds, so that well $k$ has lower bound $x=b_{2 k-1}$ and upper bound $x=b_{2 k}$, between which $V(x)=V_{r}$. The landscape is therefore piecewise constant and defined by the equation

$$
V(x)= \begin{cases}V_{\max }-d_{k} & \text { if } b_{2 k-1} \leq x \leq b_{2 k} \\ V_{\max } & \text { otherwise }\end{cases}
$$

In short, each decision alternative considered by an agent in a forced choice task is represented by a well of finite depth (efficacy) and width (generality of representation) in a one-dimensional landscape.

In the classical analog to the system we have just described, a particle in a bound state (a state having greater than chance probability of occupying an attractor well) cannot occupy the barrier regions. However, a quantum particle's distributed state occupies both wells and barrier regions. Therefore, upon measurement (collapsing the particle's state to a point), there is a finite probability that it will be found within the regions outside of the wells. These are collectively denoted as the "classically forbidden region," since this is not possible in classical systems. Measurements of a particle in this region do not contribute to the accumulation of evidence in the decision-making process, and thus their occurrence means that, on average, the system will integrate less than one bit of information per unit time. This corresponds roughly to the role of leak in classical models (e.g, [57]. In addition, the assumption of a piecewise constant landscape implies that each well is "flat"; that is, no part of a well (i.e., corresponding to a subregion of a semantic category, such as the subcategories "97\% red, 3\% yellow", and "98\% red, $2 \%$ yellow" within the category "red", in a color categorizing task) is more attractive of particles than another. This assumption can be modified for special applications, as piecewise constant landscapes can be used to approximate landscapes of arbitrary complexity and shape. The simpler form, assumed here, is known as a square well landscape for its appearance when $V(x)$ is plotted against $x$, as shown in figure 1 . 
Figure 1: An example of a square well potential landscape. The classically forbidden regions have $V_{\max }=1$, and those associated with alternatives 1 and 2 have $V_{1}=0$ and $V_{2}=0.5$ respectively.

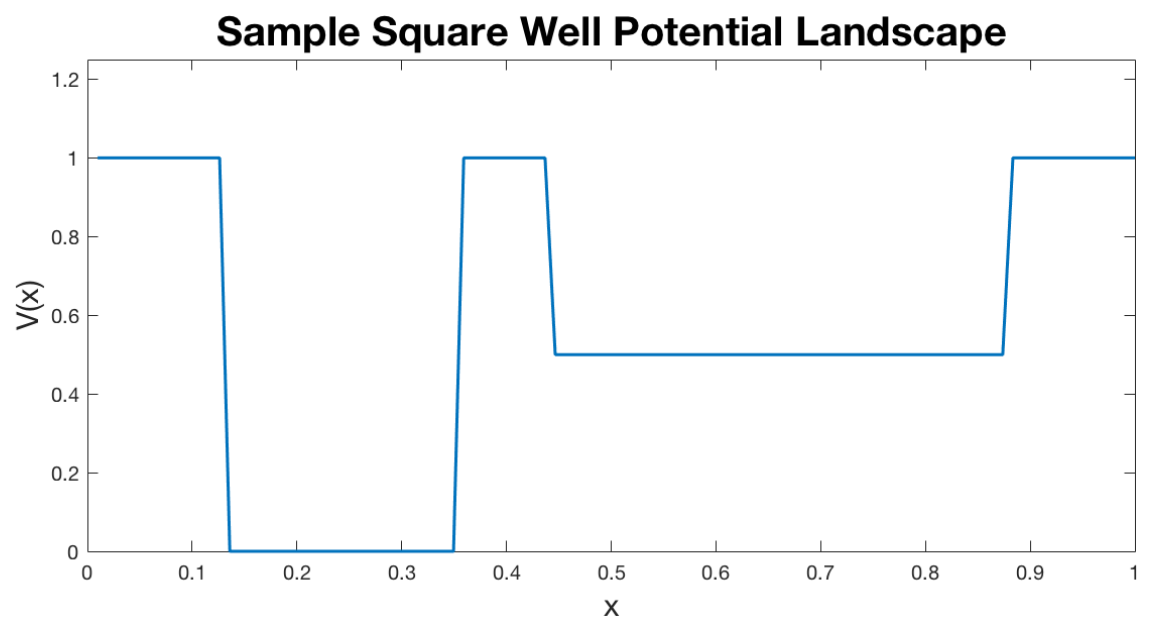

\subsubsection{The Use of the Time-Independent Schrodinger Equation and the Bound States}

Under the correct conditions, which we outline below, the Schrodinger Equation is separable into a spatial and a time component. The spatial component is aptly named the Time-Independent Schrodinger Equation (TISE), and its solutions describe the eigenstates (also called the "stationary states") of the system. The MPMW is framed in terms of the TISE, a choice based on two assumptions. The first is that, once emitted into a landscape, each particle is measured only once, and that particle is thereafter cleared from the landscape. Therefore, the time evolution of its state, had it remained in the landscape after measurement, is irrelevant. Second, although the MPMW does not assume that the landscape is perfectly static, it does assume that the landscape can be treated as static on the timescale at which measurements occur. That is, measurements are assumed to occur near instantaneously, while changes in efficacy (which can modify the landscape by influencing the depth of wells both within and between trials), representation (e.g., due to learning, which can change their width and/or separation), and arousal (which can change the energy of the system) are all sufficiently slow relative to measurement that the landscape can be treated as static on the timescale of particle measurement. Collectively, these assumptions allow MIEs to be 
computed using the TISE.

The information integration (classical) component of the MPMW only treats particles with energies that fall within a certain range, called bound states. These are the energies that may be described by some linear combination of the eigenstates of the TISE (referred to as the eigen energies), and for which the MIEs of the system are non-zero. Insofar as energy is used to model arousal, this means that the system is only able to integrate information required to perform a given task within a specified range of arousal (i.e., eigen energies). If the energy is too low (hypoarousal) or too high (hyperarousal) there is zero probability of information integration, and the task cannot be performed. Thus, use of the TISE to identify the bound states and associated eigen energies of the system provides a quantitative formulation not only of the performance of the system (MIE of the bound states) for a given energy, but also the limits of performance as a function of arousal (i.e. the range of its eigen energies).

\subsubsection{Summary of Assumptions}

To summarize, the MPMW makes the following important assumptions: first, the potential landscape is piecewise constant, implying that no region within a well (i.e., sub-component of a stimulus) is more attractive than another. Second, the distributed states of the particles may be found by solving for the bound states of the TISE, implying that individual bits of information are processed on a much faster time-scale than either adjustments in efficacy, learning or arousal. Third, in general, we focus on energies that fall within the range of eigen energies of the system (i.e., levels of arousal at which the agent can perform the task), quantitatively delimiting this range (i.e., the bounds on performance imposed by hypo- and hyperraousal) and how this interacts with predicted performance.

\subsection{The Form of the Eigenstates and their PDFs}

Given the assumptions above, we can consider the general form that the

solutions to the TISE take. The general form and its properties are true of the bound states of any system of square wells so understanding it provides an intuitive grounding for all of the effects that we discuss in later sections. 
The generalized Schrodinger Equation is given by

$$
i \hbar \frac{\partial \psi(x, t)}{\partial t}=\frac{-\hbar^{2}}{2 m} \frac{\partial^{2} \psi}{\partial x^{2}}+V(x) \psi(x, t)
$$

Where $V(x)$ depends only on space, as assumed in MPMW models, we can separate $\psi$ into its time and spatial components. The spatial component is the TISE, the eigenstates of which form the formal basis of the MPMW. It is given by

$$
-\alpha \frac{\partial^{2} \phi}{\partial x^{2}}+V(x) \phi=E \phi
$$

in which the first term is the kinetic energy of a particle, $V(x)$ is the potential energy function, and $E$ is the total energy of the system. The bound states are the solutions $E$ to this eigenvalue equation when $E<V_{\max }$. Solutions to the TISE for the form of the eigenstates can be found in [24]. In most cases, complete solutions of the TISE require the use of numerical methods. We discuss two such methods in Appendix B. Here, it is sufficient to state that the bound eigenstates for a well take on two general forms, determined by the difference between $E$ and the specific value of $V(x)=V_{r}$ for that well. Within a well where $0 \leq V_{r} \leq V_{\max }$, the solutions have the form

$$
\phi(x)=\left(c_{1}+c_{2}\right) \cos (\sqrt{k} x)+i\left(c_{1}-c_{2}\right) \sin (\sqrt{k} x)
$$

The state functions given here, which have both positive and negative components and have imaginary parts, are distinguished from the particle's PDFs by these properties. However, the PDF, which we introduce shortly, is derived from the state functions, so understanding them is of critical importance.

Within the classically forbidden region, where $V=V_{\max }$ and $\left.E-V_{(} x\right)<0$, the eigenstates have the form

$$
\phi(x)=c_{3} e^{-\sqrt{\kappa} x}+c_{4} e^{\sqrt{\kappa} x}
$$

where the equations are a composition of exponentially growing and exponentially decaying functions. In both cases, the constants are determined by the boundary conditions for the TISE, which can also be found in [24]. For the bound states, the boundary conditions require that the state of the particle decays to zero at the outer edges of the landscape, as $\phi \rightarrow \pm \infty$. This is equivalent to requiring that the particle exists somewhere in an infinite space. In between wells, however, $\phi$ simply decays as it moves away from the well 
boundaries, meeting itself smoothly in between. The primary importance of the form of $\phi$ anywhere outside of the wells is that it is non-zero everywhere. This property provides access to the classically forbidden region. In the MPMW, access to the classically forbidden region means there is a finite probability of "dropping a bit," analogous to "leak" in classical models. To help visualize the general forms of the bound states, an example of a two well landscape and one of the solutions for $\phi$ is shown in figure 2 .

Figure 2: An example of a bound state and its generating potential. Note that the visual representation of the bound state is not truly smooth. This is due to the discrete method used to compute $\phi$ (see, B), which effectively segments the resulting state functions into very small straight lines, creating small errors around turning points of the state function.

\section{An Example Particle Bound State Function with Generating Potential}

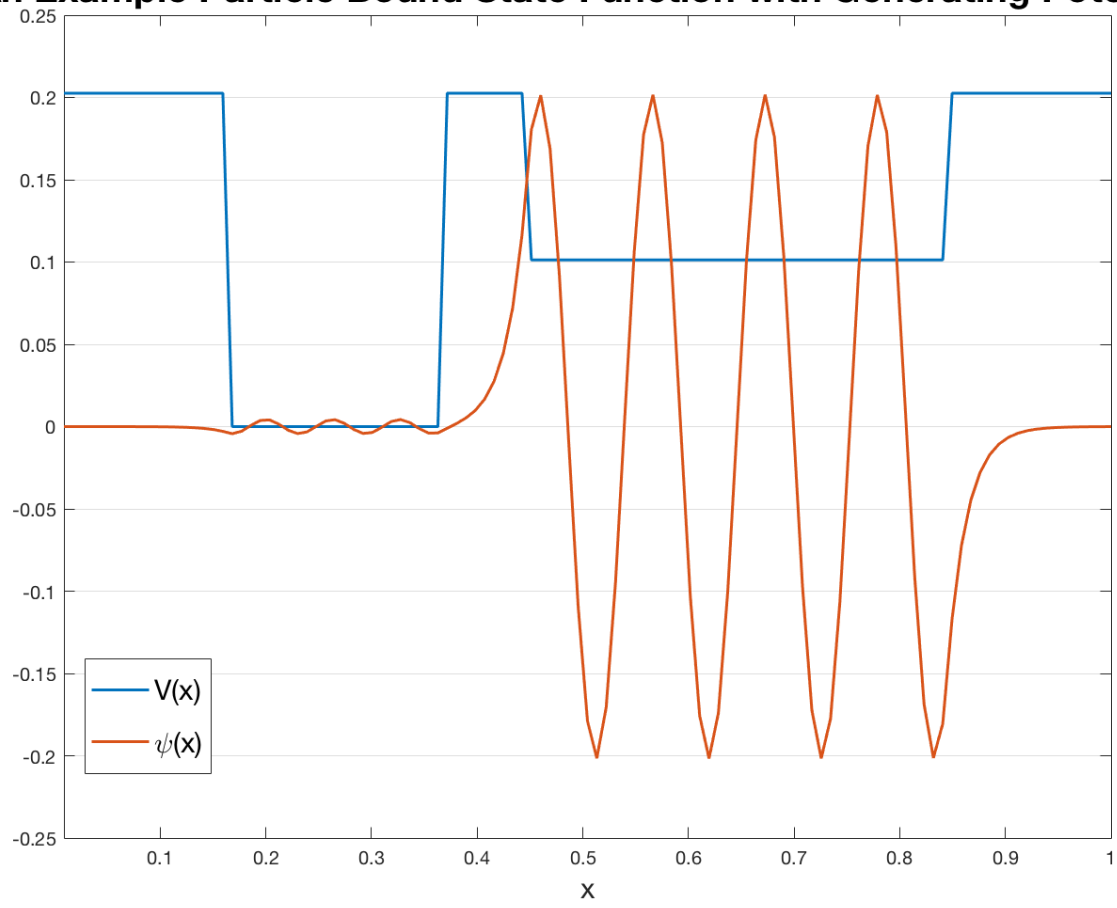

As noted above, $\phi$ has both positive and negative values as well as real and imaginary parts, which means that it is not a PDF. Instead, the PDF of a bound state is given by the L2-norm (the continuous equivalent to an inner product) of $\psi(x)$,

$$
\operatorname{PDF}(x)=\psi^{*}(x) \psi(x)
$$


- This PDF describes the probability that a particle will be measured to be in some region within the landscape, from which we may find the MIEs of a system. The PDF (and therefore $\psi(x)$ ) are bounded such that

$$
\int_{-\infty}^{\infty} \psi^{*}(x) \psi(x) d x=1
$$

by the requirement that the particle exists somewhere within an infinite landscape. An example of a PDF with its generating state function is shown in figure 3. Note that the functional form of the PDF is very similar to that

Figure 3: An example of a bound state in a double well potential and its resultant PDF.
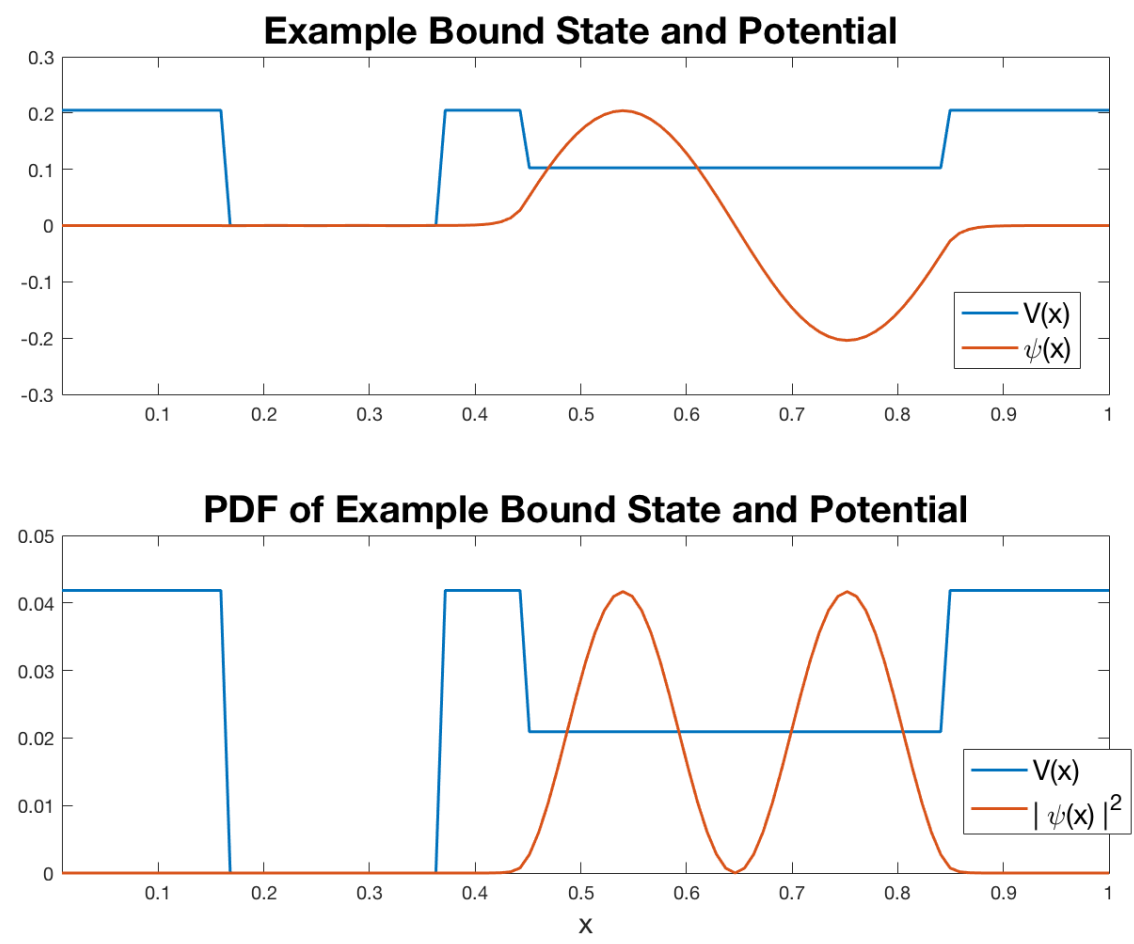

of the state function, but that the PDF is strictly positive. Additionally, because the norm of a complex function is formed from the original function and its complex conjugate, the PDF of $\phi(x)$ has no imaginary parts.

In order to find the MIE for a well $k$ in a given state $j, M_{k, j}$, we take the integral of the PDF from one boundary of that well to the other (that is, we 
find the area within it).

$$
M_{k, j}=\int_{b_{2 k-1}}^{b_{2 k}} \psi^{*}(x) \psi(x) d x
$$

Where $k$ is the index of the well. Solving this integral requires finding each of the constants that describe the different components of $\phi$ and must be performed numerically for most potentials (see Appendix B).

Summary The following general properties of the PDFs characterize properties of the MPMW. First, PDFs are generally larger for the regions within a well, so a particle is generally more likely to be found within a well than outside it. This means that, as long as some finite amount of efficacy is allocated to a stimulus or task (i.e., its depth is non-zero) and, as long as arousal falls within the bound states, an agent is more likely to integrate task-relevant information than not. Second, outside of the wells, the PDF decays exponentially as it moves away from the boundaries of a well, meaning that a particle is much more likely to be found in the regions immediately surrounding the wells than at the far reaches of a landscape. This also implies that wells that are closer in proximity to one another will exhibit greater conflict than those that are further away. In section 6 we illustrate this for a double well (2AFC) context. Third, the PDF is non-zero everywhere, meaning that the particle may technically be found anywhere in the landscape at any time. This access to the "classically forbidden region" of the landscape in the MPMW allows for the "dropping bits", with an effect similar to leak in classical formulations (e.g., [57]). In the MPMW, this means that it is always possible for a bit of information to be integrated as evidence in favor of the non-target stimulus, or fail to be integrated in a way that is relevant to the current task at all, regardless of how salient the target stimulus is or how closely an agent attempts to attend to it.

\subsubsection{Combined States}

As noted earlier, the state of an unmeasured particle contains all information present in the environment, some of which is lost by measurement. More specifically, if the energy of the particle falls within the range of energies of its bound states, then its initial state is describable by a linear combination of eigenstates. Measurement of a particle in one of these "combined states," 
collapses it to one of the contributing eigenstates. Note that while the PDFs of the individual eigenstates do not change with time, their stationary nature relies on the separability of the time and space components of the Schrodinger Equation. In contrast, the PDFs of combined states do change over time and, therefore, so do their MIEs. Thus, when considering a single particle, the time-varying nature of the combined state, and consequently the MIEs of the wells (i.e., decision alternatives) is an important determinant of the integration process.

To better understand this property, consider an agent occupying a state with some energy that falls between the energies of eigenstates $E_{j}$ and $E_{j-1}$. This energy can be described by

$$
E=a E_{j}+(1-a) E_{j-1}
$$

and its state by

$$
\Psi=(\sqrt{a}) \psi_{j}+(\sqrt{1-a}) \psi_{j-1} .
$$

The PDF of this state will oscillate with frequency $E_{j}-E_{j-1}$ and mean

$$
\mathbb{E}(M)=a M_{k, j}+(1-a) M_{k, j-1} .
$$

It is interesting to note that combined states and their oscillating PDFs can be used in a single particle model to describe multi-stable perception, although this is outside the scope of the present article. In the present context, there are two things that are critical to note. First, in a standard MPMW model, the expectation of the MIE $\mathbb{E}(M)$, is sufficient to describe the information integration process. Second, unlike in classical systems, when the time varying combined state of a quantum system is measured (i.e., the information contained in a particle, such as its position), the measurement causes it to collapse into only one of its (stationary) eigenstates. For example, imagine a ball that is rolling about on a table. The table can be described by a plane and the position of the ball can be described entirely by a pair of $\mathrm{x}$ and $\mathrm{y}$ coordinates (analogous to a combined state, with the $\mathrm{x}$ and $\mathrm{y}$ axes corresponding to the eigenstates of the system). In a classical system, a measurement of the ball's position can recover both its $\mathrm{x}$ and $\mathrm{y}$ coordinates and doing so does not change them. By analogy, in a quantum system, observing the ball would force the ball into a position described by only the $\mathrm{x}$ or only the $\mathrm{y}$ axis. While not exact, this analogy conveys the idea that when a measurement is made on a quantum system in a combined state, the 
output of the measurement will not reflect a combined state, but rather only one of the states contributing to the combined state. If we take a very large number of particles all prepared in the same combined state comprised of $\psi_{j}$ and $\psi_{j-1}$, the mean probability of measuring one of those particles to be in well $k$ is given by equation 12 .

To summarize, the bound state of any system in which the TISE is applicable will be a linear combination of the eigenstates found by solving equation 4. Measurements of these states will reflect the eigenstates contributing to a system, and therefore the MIEs of a system can be defined only in terms of the MIEs of the eigenstates,

$$
M_{k}=\sum_{j=1}^{J} \alpha_{j} M_{k, j}
$$

where $k$ is the index of the well and $j$ are the indices of the eigenstates.

Having characterized the general forms of the states and how to find the corresponding MIEs, we now consider how the parameters of wells affect the MIEs. These effects characterize how factors such as efficacy, the generality with which stimuli are represented, and the energy of the system impact the information integration process, analogously to changing the drift rate and variance in a classical system. In the following sections, we first consider single and double well landscapes (corresponding to signal detection and simple 2AFC tasks, respectively), and then generalize the results to a multi-well landscape (corresponding to multialternative decision making) and consider the effects of energy.

\section{Fundamentals of Eigenstate Distributions}

For every landscape we will consider, the number of eigenstates, their energy relative to well depth, the extent of their localization, and to which well they are primarily localized are fundamental properties in determining how task performance changes as a function of arousal. In this section, we address the most general properties of eigenstates: their maximum and minimum energies relative to well depth, their number, and how these relate to performance. 


\subsection{The Ranges of Hypoarousal and Hyperarousal}

We illustrate this discussion by explicitly considering the single well landscape, as it is one of few for which the TISE can be solved analytically (see [24] for a tutorial). However, it is worth noting that the general properties of the eigentstates of a single well landscape also hold true for multi-well landscapes. Thus, as we address the basic properties of eigenstates in a single well context, they provide an intuitive grounding for similar properties of multi-well landscapes, of which the single well is simply a special case.

The single square well potential is defined by a simple piecewise function,

$$
V(x)= \begin{cases}0 & \text { if } 0<x<a \\ V_{\max }<\infty & \text { elsehwere }\end{cases}
$$

And the eigen energies of the system are given by the solutions to the transcendental equations (see [24] for derivations):

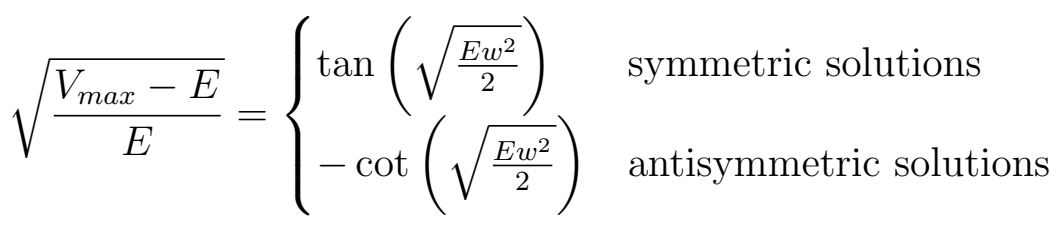

which depend on both the depth $\left(V_{\max }\right)$ and width $(w)$ of the well, with only $E$ as an unknown.

Note that, regardless of depth and width, $E=0$ is never a solution to these equations. This implies that there is a range of energies between the bottom of the well and the lowest energy bound state (the ground state) that cannot be described by a linear combination of bound states and is, therefore, not within the range of states admitted by the well. This range of energies is denoted the range of hypoarousal, and a particle in this energy range will not be found within the well. We interpret this as a range of energies in which an agent is unable to attend the target stimulus and, therefore, cannot perform the task due to low arousal. Additionally, while there are periodic depths and widths at which $E=V_{\text {max }}$, because of the finite well depth that we assume for these landscapes, there is also an infinite range of energies exceeding $V_{\max }$, at which task-relevant information is only integrated at chance $(0$, in an infinite landscape). This is the range of "hyperarousal", a range interpreted as one in which the agent is unable to integrate information due to distraction or agitation. The hypo- and hyperarousal states impose a "working range" of 
energies, in which an agent is able to integrate task-relevant information. They are bounded below by the ground state and above by the most excited state, which, in turn, are determined by the depth and width of the well.

Within the working range of energies, performance (determined by MIE) varies as a function of energy. In the remainder of this section, we discuss how energy determines MIE in preparation for the remainder of the article, wherein we discuss the details of how well parameters effect hypo- and hyperarousal and performance.

\subsection{Well Parameters Impose a Discrete Threshold on Integration}

To understand the effects of well parameters on the working range and performance, it is helpful to understand their effects on the number of states the well admits. For a single well, this number is given by a simple equation,

$$
N=\left\lceil\frac{w}{\pi} \sqrt{2 V_{\max }}\right\rceil
$$

In more complex landscapes, equation 16 can be used to estimate the number of eigenstates admitted by the landscape, but where $w$ is the sum of the widths and $V_{\max }$ is the maximum depth over all wells in the landscape.

Note that $N$ increases as the step-wise square root (sublinearly) of depth and stepwise linearly in width. Note also that $N$ is never zero, implying that every well admits at least one eigenstate, so, where efficacy is non-zero, there must exist at least one state of arousal in which information integration can occur. However, where $N=1$, an agent's arousal must occupy an exact energy in order to integrate task-relevant information. Deviation from this energy by any amount will result in entering either a hypo- or hyperarousal state. Thus, where $N=1$, the working range of energies is infinitesimally small, effectively zero. For this reason, and under the assumption that arousal energy is not perfectly static, we assert that an agent will only be able to perform a task or respond to a stimulus where efficacy is sufficient that $N=2$. Furthermore, the amount of efficacy required for this increases exponentially as stimulus generality decreases linearly.

This assertion comes with the significant implication that, for every well, there will be a specific well depth at which a stimulus transitions discretely from being non-integrable to integrable. This corresponds to a discrete psy- 
chophysical thresholds for stimulus detection, for each integrator, that can be "crossed" by increasing stimulus salience, allocation of attention, or experience with the stimulus (automaticity of the corresponding task), all of which contribute to the efficacy of processing a stimulus, modeled in the the MPMW framework as well depth. Accordingly, the MPMW predicts that the transition from undetectable to detectable occurs discontinuously at a depth threshold specified by the well width, rather than gradually, with the increase in any of the factors listed above. However, it is important to note that this threshold may be specific to individual integrators (which may be a neuron or pool of neurons), as each will have some distinct level of sensitivity to a stimulus that impacts the dimensions of a well or wells associated with that landscape. It is possible that the most accurate model of such an effect would consider an aggregate of integrators with landscape dimensions that fall within a range. In this case, the MPMW would predict a sort of "fuzzy threshold" for stimulus detectability for a population that then translated into a bottleneck for reportability dependent upon propagation properties. This form of bottlenecking has been described in the work of Van Vugt et. al. [59].

The MPMW framework predicts a similar thresholding effect with respect to the generality with which an expected stimulus is represented that determines whether or not it will be detectable. That is, for a given energy (level of arousal) and well depth, there is a precise width at which a particle will discontinuously transition from unidentifiable (when a well is too narrow, and the working range infinitesimal) to identifiable (the well is wider and the working range finite). At this point, as the working range becomes finite, the integration efficiency (MIE) transitions from $M=0$ to $M>0$.

To illustrate this effect, consider the case of detecting the signal at a pedestrian cross walk discussed earlier and how the color of the signal to be detected is represented. If it is too narrowly defined (well width too narrow), such as a very specific shade of green, no evidence will be integrated despite the presence of a signal. The MPMW predicts that there is a precise level of generality (well width), at which the signal will transition discontinuously from non-integrable to integrable. Of course if representation is insufficiently constrained (too wide), it may result in the integration of other colors present in the environment (i.e., such as a green traffic light) despite their irrelevance to the task, resulting in false-positive responses. 


\subsection{Eigennumbers as a Shorthand for MIEs}

In addition to its energy, each eigenstate of a well is associated with an eigennumber. $E_{1}$ denotes the energy of the ground state and $E_{N}$ denotes the highest energy eigenstate. As discussed above, these energies comprise the upper bound of the range of hypoarousal and the lower bound of the range of hyperarousal respectively. When changes to a well's parameters increase the number of eigenstates it admits, we will refer to the eigenstates with eigennumbers exceeding the previous largest eigennumber as "new" eigenstates. Note, however, that according to equation 15 the energies of the "preexisting" eigenstates (the ground state, for example) also change as a function of well parameters, although they retain their overall shape (number of peaks) and relative performance properties, despite changes in their energy.

The eigennumber of an eigenstate can be used to roughly characterize performance of the system for arousal in that range. Lower eigennumbers denote lower energy eigenstates, which are more localized to the well (or, in a multi-well system, a well) and therefore have a larger associated MIE than higher energy states. That is, a lower energy eigenstate will be associated with higher performance than its higher energy counterparts within a well of constant depth (efficacy) and width (generality). This result is in keeping with findings that an increase in arousal resulting from a shift away from the phasic mode of LC-NE activity and toward the tonic mode will be associated with greater levels in distractibility or an inclination toward disengagement from the task [58].

To gain an intuition for the physical interpretation of the effect of increasing eigennumber, consider the classical analog: a ball rolling in a landscape, in this case with a single valley. One moving at a lower speed (lower kinetic energy) is both more likely to fall into a valley (rather than skipping over top of it) and will settle at its base more quickly. Although a quantum particle cannot be said to ever truly "settle into" a well, lower energy has the analogous effect of increasing the rate of decay of the particle's PDF outside of the well and increasing the probability that the particle is found within the well (i.e., its MIE). This effect is illustrated in figure 5, that shows the derivative of MIE in the working range of a single finite well plotted against fractional arousal $(E / d)$ for systems of varying depth and width. Note that, for all systems, the derivative is always negative and decreases monotonically as energy increases, indicating that MIE decreases with associated increases in arousal. Note also that figure 5 illustrates the subtler effect that, in gen- 
eral, deeper wells are associated with higher MIEs across their full working energy spectrum.

Taken together, these observations reveal an important prediction of the MPMW framework: increasing efficacy (depth), and thus the number of a well's low energy eigenstates, produces an increasing "all or nothing" effect, such that states localized to a well remain closer to the the ground state of that well, and are associated with correspondingly higher MIEs across the full working range. As a result, the agent is likely to exhibit either high levels of performance (within the working range of arousal), or to fail to perform entirely (falling within the ranges of hypo- or hyperarousal).

In summary, the MPMW makes two major predictions about how MIE behaves as a function of arousal in a single static well. First, performance is optimized by maintaining the lowest possible arousal without entering the range of hypoarousal (i.e., maintaining energy that exactly matches the ground state energy of the system). Second, deeper wells (i.e., higher efficacy) will exhibit a larger performance discontinuity as a function of arousal. That is, they will exhibit higher MIEs (i.e., show less sensitivity to increases in arousal) across the working range of energies, resulting in a more precipitous drop off when energy exceeds the threshold for hyperarousal. This discontinuity in performance from very high to very low in deeper wells will appear more pronounced in comparison to shallower wells, for which the deterioration of performance (i.e., decrease in MIE) as arousal increases is at once more gradual but more substantial.

\section{$5 \quad$ Single Well Landscapes}

In this section, we begin our analysis of well parameters and their impact on MIEs with landscapes containing a single well of finite depth. Such a landscape can be used to model simple signal detection tasks, such as monitoring for the appearance of the "cross" signal at a pedestrian crosswalk. In this context, the agent is faced with the task of integrating a stream of information from a single stimulus that changes discontinuously from one static state to another. We can model such a process with a system in which the agent integrates a value representing the difference between the signal and a stored value for the signal's default. When the signal is in its assigned default state (i.e., absent), the landscape is flat (the depth of the single well is $V_{\text {well }}=0$, corresponding to the absence of any signal salience), and when 
Figure 4: MIE v fractional energy (energy/depth) for wells at different widths and depths. Note that MIEs are generally larger and decrease less as a function of fractional energy for deeper wells
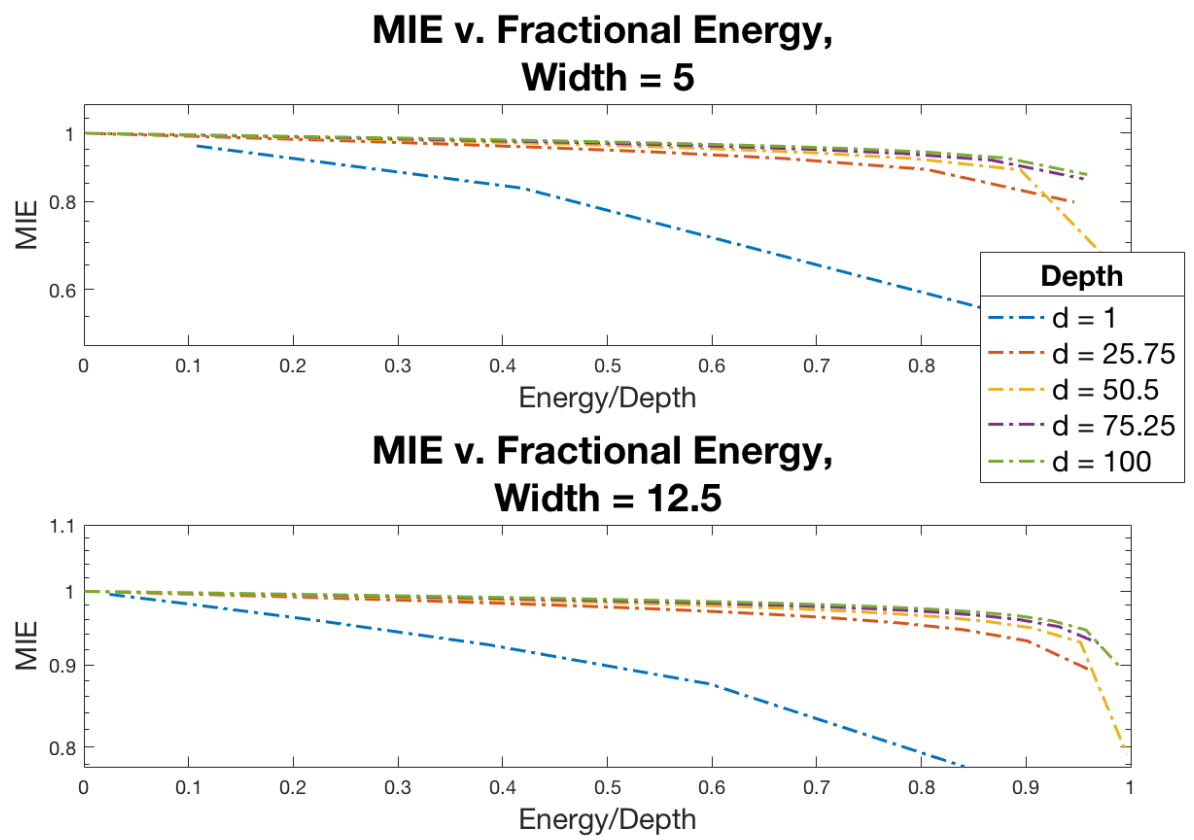

MIE v. Fractional Energy,

Width $=20$

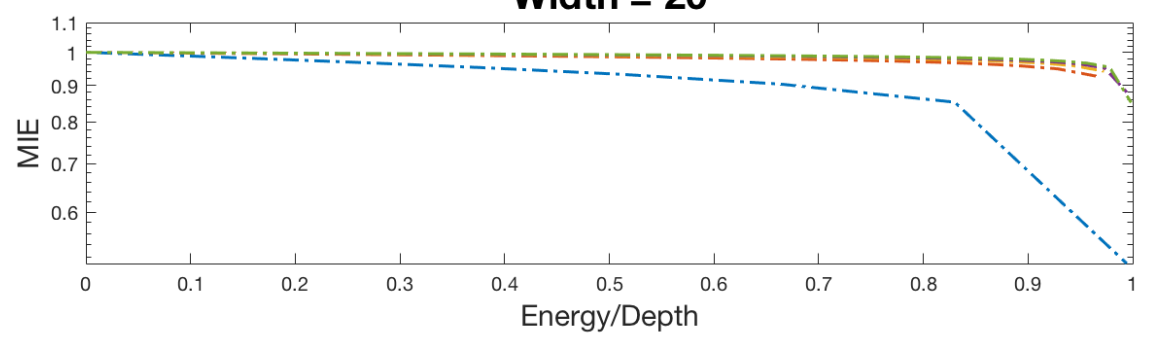

the signal changes (becomes salient), the depth of the well changes to some finite value $V_{\text {well }} \neq 0$. At each time step, the agent takes a measurement of a particle's position (representing a bit of information) and, if it is measured within the well, it is integrated as a bit of information in favor of the signal's detection, and the decision to act accordingly. 
Figure 5: Derivative of MIE as a function of its fractional energy (energy/depth). Note that all derivatives are negative, indicating that MIE decreases as energy increases. Note also that all derivatives are decreasing, indicating that MIEs decrease more rapidly as energy increases.

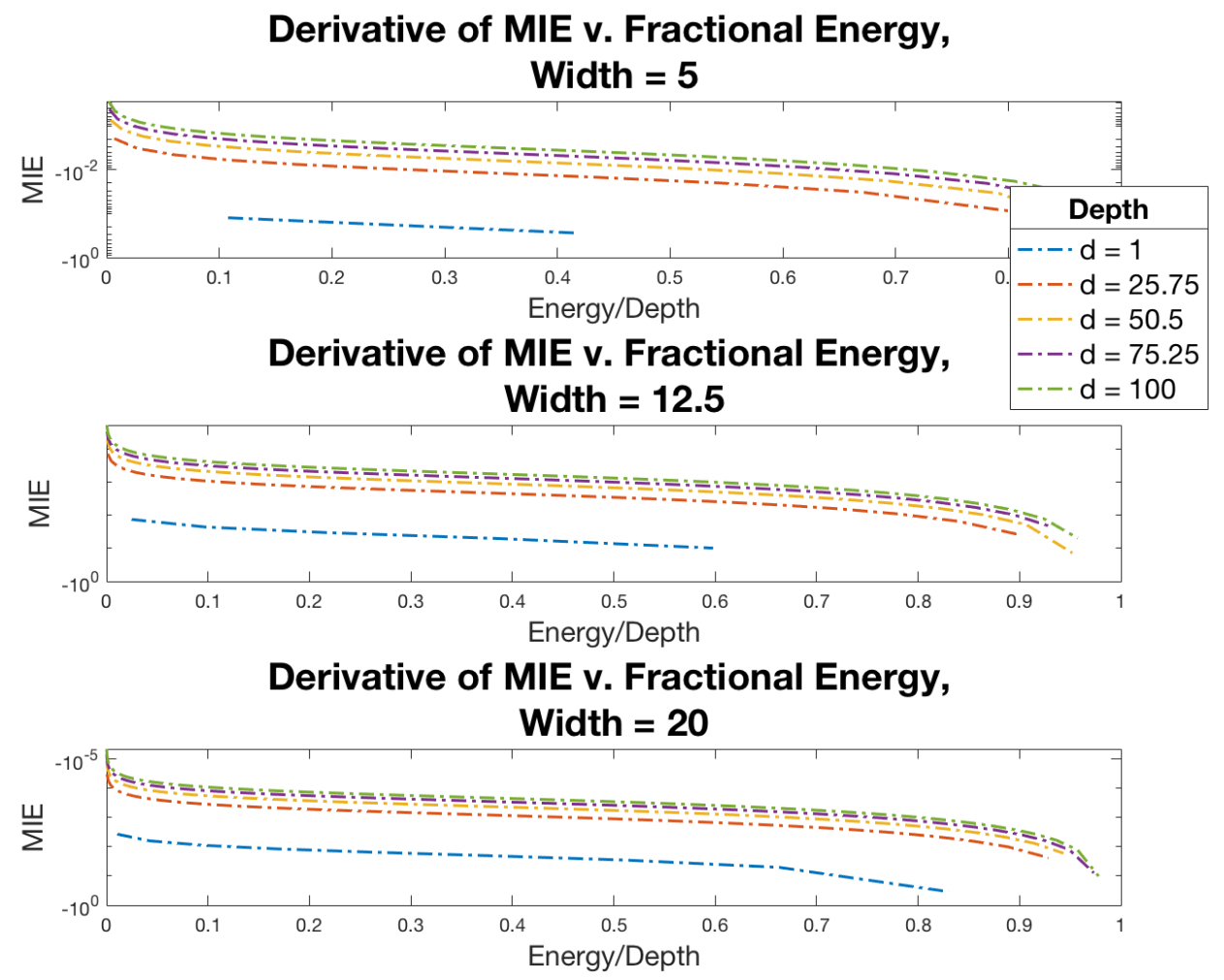

Note that the finite depth of the well denotes a non-zero probability that a particle will not be measured within the well, but in the classically forbidden region, corresponding to a "dropped bit". Furthermore, finite depth denotes that the probability of measuring a particle in the well is sensitive to the energy of the system (i.e., arousal). In the remainder of this section, we address how the parameters of a single well, its width and depth, affect the eigenstates of its landscape, providing a grounding for considering such effects in more complex, multi-well systems further on. 


\subsection{Effects of Depth on Eigenstates and Performance}

Increasing Depth Increases the Ground State Energy As demonstrated by equation 16, increasing well depth introduces new eigenstates. Meanwhile, existing eigenstates occupy higher energy than they previously did, thus increasing the range of hypoarousal. The range of hypoarousal is, however, asymptotically bounded by the ground state of an infinite depth square well of the same width (given by $\pi^{2} / 2 w^{2}$ ). That is to say that, increasing efficacy increases the ange of energies at which it is not possible to integrate information due to low arousal. However, because the increase is limited, energies exceeding the infinite-depth ground state will never enter a state of hypoarousal as efficacy increases. Furthermore, as depth increases, the most excited state also increases in energy, incorporating higher levels of arousal into the working range. These points are illustrated by figure 6 , that plots eigenenergy against eigennumber for a range of depths of a finite well.

As discussed in section 4, the ground state of a single well landscape is associated with the highest MIE and thus highest performance. This, coupled with the increasing ground state energy associated with increasing well depth, implies that an optimal agent will demonstrate an increase in arousal commensurate to an increase in efficacy (as by the allocation of selective attention). Furthermore, as shown in figure 7 , this increase will be greater for stimuli with a less general representation. That is, the greater precision required for accurate stimulus representation, the greater increase in arousal required to maintain optimal performance at a given efficacy as efficacy increases.

Increasing Depth Produces Periodic Discontinuous Increases in the Working Range As shown in Figure 7, the ranges of hypo- and hyperarousal increase overall with well depth (efficacy). Despite this, the working range of energies also increases with well depth. This is because the rate of increase of the breadth of energies outside the working range is less than that of the total depth of the well, so that the working range of energies occupies a progressively larger fraction of the total range of bound energies.

The increase in the working range exhibits periodic "quantum leaps" with continuous increases in depth, corresponding to the addition of new eigenstates to the system. As predicted by equation 16, the rate at which these "quantum leaps" occur decreases as depth increases. This effect may have normative consequences, as it predicts a reduced return on performance- 
Figure 6: Graph of eigenenergy across several depths (in a well of width w $=5$ ). Note that, as depth increases, the energy associated with the most excited eigenstate (highest eigennumber) grows, expanding the total range of energy associated with the well. This implies that both the range of hypoarousal and the "working range", the range of energies between hypo and hyperarousal, grow as the well becomes deeper.

\section{Eigen Energy V. Eigen Number, Several Depths}

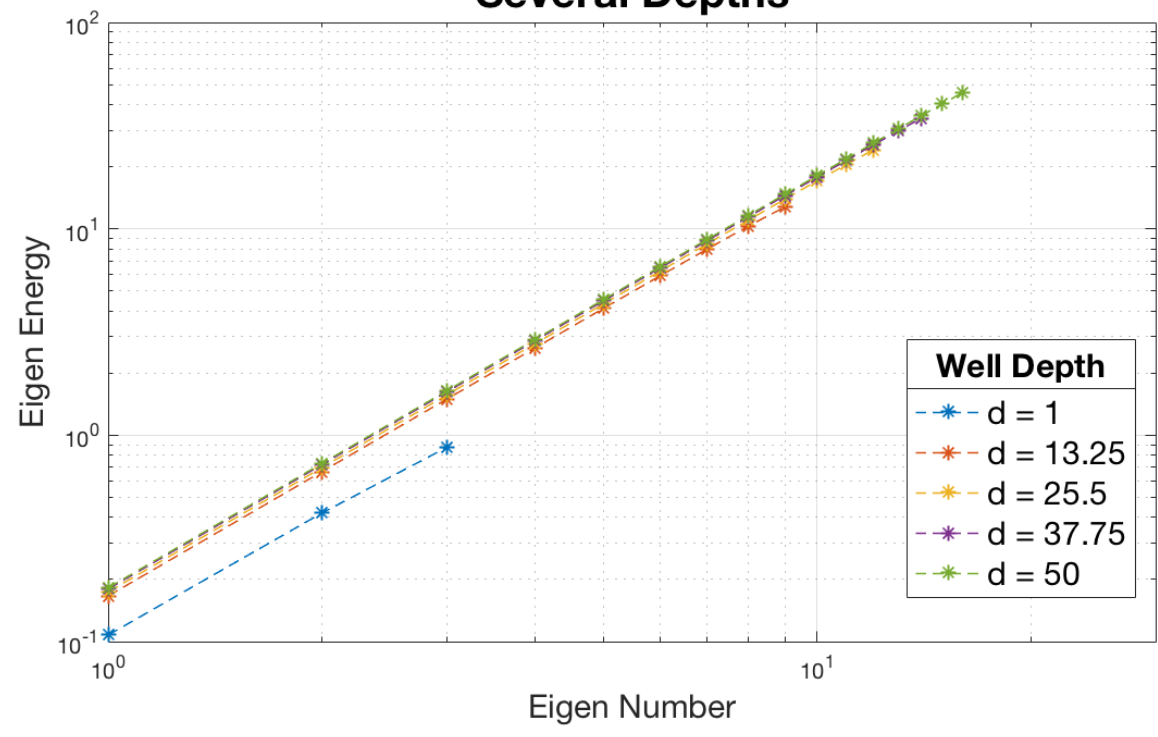


Figure 7: Top Left: Range of Hypoarousal versus depth at several widths. Log-Log scale. Range of hypoarousal is overall increasing in depth and larger at narrower widths. Nonmonotonicities are attributable to the introduction of new eigensates and are most pronounced in narrower wells. Bottom Left: Range of Hyperarousal V. Depth at several widths. Log-Linear scale. Range increases until a new eigenstate is introduced, then drops precipitously. These drops occur approximately as the square root of depth. Hyperarousal range is overall increasing in depth and decreasing in width, with range converging as width increases. Top Right: Working Range V. Depth at Several Widths. Working range increases approximately linear with large increases associated with new eigenstates. At very large width, working range converges to depth, causing it to increase linearly with smaller stepwise effects. Bottom Right: Fractional Working Range V. Depth at several widths. Working range/depth increases depth increases, saturating at 1 . That is, despite the growth of the ranges of hypo and hyperarousal, growth of depth outpaces them. Larger working ranges/depth are associated with larger width.
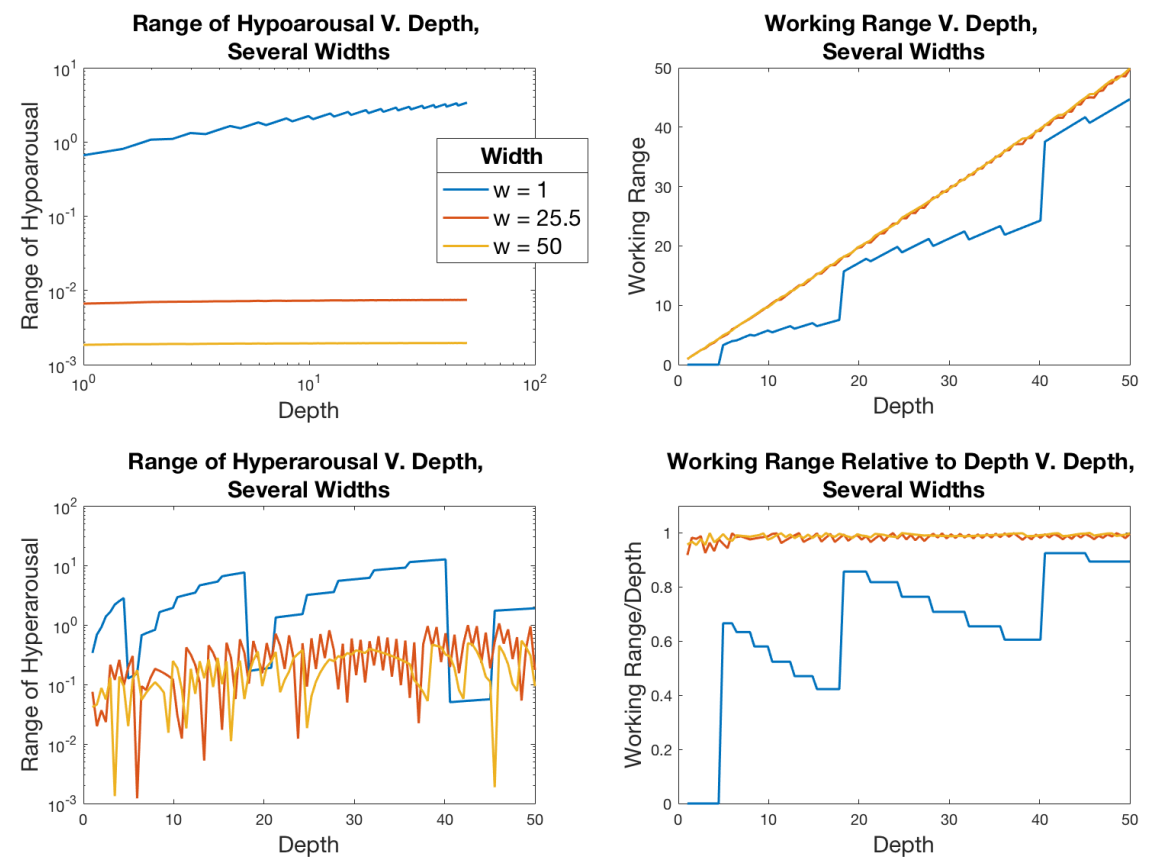
related parameters with increased efficacy (as by allocation of controlled attention), that in turn could be signaled as a cost for such investment (experienced as "mental effort"). While mechanisms for the signaling of such costs have been proposed within the context of classical models (see, [16], [51], [38], [7]), it is an emergent property of the MPMW framework. Furthermore, the MPMW makes the unique prediction that agents may be disposed to increase control in quanta of exponentially increasing magnitude (following the decaying frequency with which new eigenstates appear), rather than continuously, to capitalize on the quantum leaps in working range.

Increased Efficacy May Produce Hypoarousal For an agent statically occupying the correct range of energies, increasing efficacy will result in improved performance. Such improvements require that the state of arousal falls within or enters into the working range of energies. As discussed above, the ground state energy of a well of fixed width increases asymptotically as efficacy increases. Therefore, where arousal is less than the asymptotic value of ground state energy $\left(E<\frac{\pi^{2}}{2 w^{2}}\right)$, increasing stimulus efficacy will eventually drive that energy into the range of hypoarousal, in which the task can no longer be performed. This effect may manifest as boredom resulting from a task becoming insufficiently challenging, as the applied efficacy exceeds task requirements sufficiently to produce disengagement. As an optimal agent will seek to maintain a ground state that matches their arousal, this effect predicts that an optimal agent that cannot increase their arousal will instead decrease selective attention to a stimulus as it becomes more salient, balancing the combined effects to avoid hypoarousal.

Where arousal is lower than the asymptotic value of ground state energy and efficacy is sufficiently low that arousal is within the working range, performance will increase as a function of increasing efficacy. However, as efficacy continues to increase, performance will begin to "flicker" on and off in keeping with the non-monotonicities that occur in the ground state as it exceeds the threshold for hypoarousal (see figure 8 above). At efficacy immediately below the point at which arousal begins to fall into the range of hypoarousal (begins to "flicker"), performance will peak as arousal matches the ground state. Increasing allocated efficacy beyond the point at which "flickering" begins to occur will be counterproductive, as the the task will be performable at progressively narrower ranges of depth. In these ranges of depth with non-zero MIE, progressively smaller fluctuations in efficacy will 
cause the MIE to drop to zero, and the agent to be unable to process the stimulus.

Therefore, the MPMW predicts that, for a well of fixed generality and static arousal less than the maximum ground state for that generality, there are three distinct ranges of efficacy: At too little efficacy, the agent will be in a state of hyperarousal and therefore be unable to perform the task. This is the case where the task is simply too difficult for the agent to perform without increased attention or salience, making them highly distractible. At too much efficacy, the agent will be in hypoarousal and performance will "flicker" on and off until finally "flickering off" permanently. This is the case where the task is too easy or the agent has managed to allocate too much attention, becoming under-stimulated. Between these ranges, the agent will be within the working range and able perform the task at high MIEs. Finally, performance will peak at the highest efficacy that falls within the working range, at which point arousal will match the ground state energy of the landscape. This implies, under the constraints stated above, an optimal efficacy, above which performance deteriorates.

Hyperarousal May be Overcome by Increased Efficacy In contrast to its counterintuitive effects with respect to hypoarousal, increased efficacy is highly effective in mitigating the effects of hyperarousal. As shown in figure 8, increasing efficacy to encapsulate high arousal in the working range will produce discontinuities in improvements of performance. Improvements stabilize once the energy of the most excited state permanently exceeds the state of arousal, so that arousal remains within the working range as the well continues to deepen. After this point, while increasing efficacy will continuously improve performance, the rate of improvement is decreasing, so that there are diminishing returns on increased efficacy. These are the familiar saturating effects of increasing controlled attention, salience, and automaticity.

In order to maintain consistently present performance, a constant-generality stimulus will require both an arousal level that exceeds the maximum ground state energy, and sufficient efficacy that the energy of the maximum excited state consistently exceeds arousal. Within these parameters, increasing efficacy will improve performance, but with diminishing returns as MIE gradually approaches its asymptote of 1 , exhibiting small "quantum leaps" along the way. 
Figure 8: Mean MIE as depth increases for agents with constant arousal. The top image is for arousal lower than the final ground state of the system. The middle image is for an agent with arousal just above the final ground state of the system. The bottom image is for an agent with arousal above the initial most excited state of the system. Note that, in all cases, more salient stimuli have higher MIEs, indicating that generality increases efficacy of efficacy.
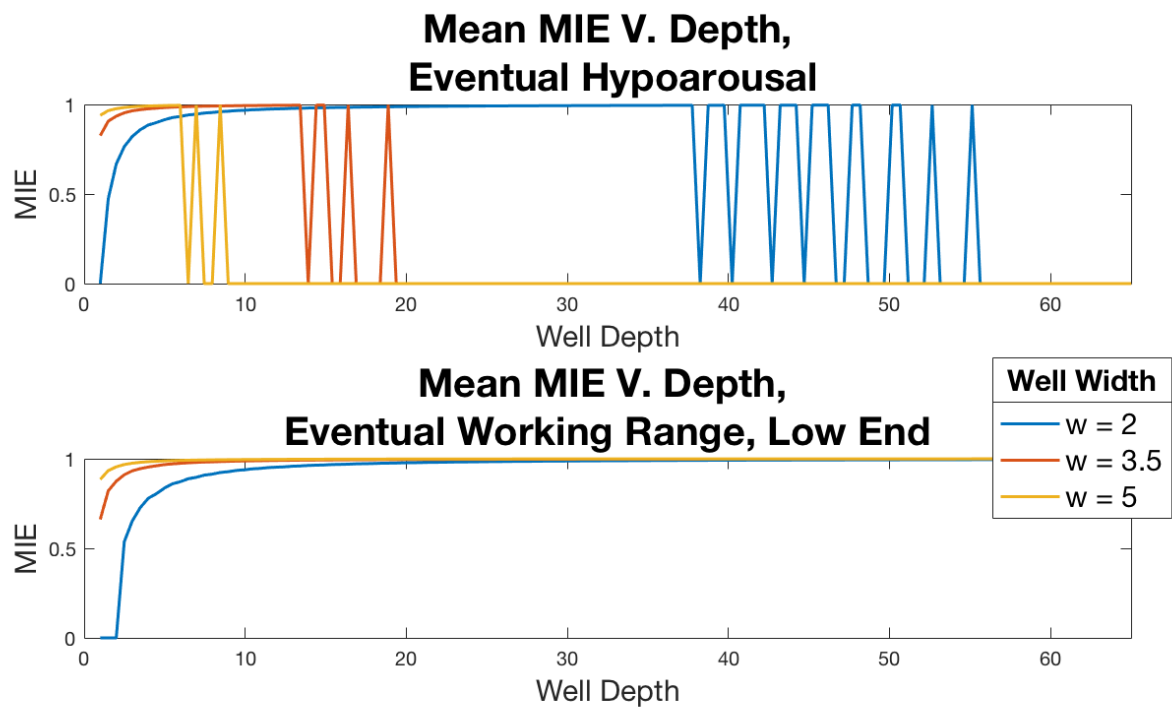

Mean MIE V. Depth, Eventual Working Range, Initial Hyperarousal

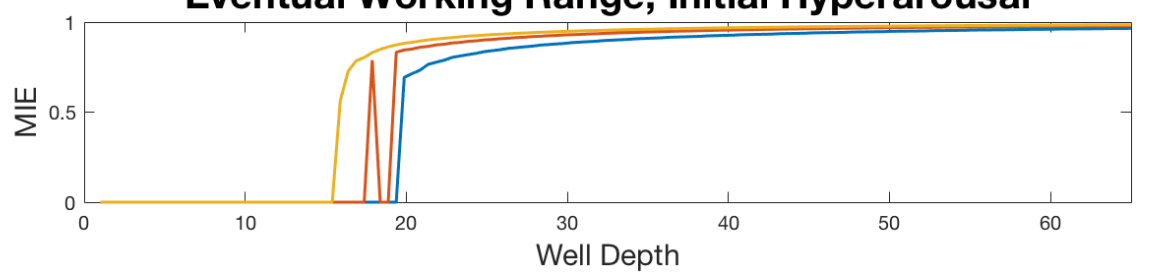


Conclusion: The Effect of Depth on Performance Parameters In conclusion, the MPMW makes several predictions with normative implications surrounding increased efficacy in single well landscapes. First, the MPMW predicts that depth increases for constant-width wells will result in increases in the working range of energies that exhibit periodic "quantum leaps". These quantum leaps may motivate discrete increases in efficacy. Furthermore, depth increase is accompanied by asymptotically increasing ground state energy, the energy at which performance is optimized. Therefore, an optimal agent will exhibit asymptotic arousal increases accompanying with linear efficacy increases. These increases will be greater for stimuli of less general representation. Finally, the MPMW predicts that an agent needing to avoid hypoarousal may do so by avoiding increasing efficacy and one needing to overcome hyperarousal may do so by increasing efficacy. However, as the range of hyperarousal is overall increasing as a function of well depth, overcoming linearly increasing hyperarousal requires greater-than-linear efficacy increase. Finally, performance increases that accompany increasing efficacy are asymptotic, and the diminishing returns may result in increasing felt effort with linear increases in efficacy.

\subsection{Effects of Width on Eigenstates and Performance}

In addition to the effects of increased well depth, there are similar but differentiable effects that arise as a result of increasing well width, mapped by the MPMW to representational generality. In the remainder of this section, we explore the effects of increasing well width on single well eigenstates and their implications for performance.

Increasing Width Increases the Working Range of Energies Similarly to increasing depth, and as shown by equation 16, increasing well width linearly has the effect of adding new eigenstates. In contrast to increasing depth, however, these new states appear with constant frequency as a function of linearly increasing width. Furthermore, increasing width causes existing eigenstates to occupy lower energies than previously, thus decreasing the range of hypoarousal. This effect is illustrated by figure 9 , that plots eigen energy against eigennumber for a range of widths of a finite well. This plot shows that increasing well width has the effect of increasing the working range of energies, implying that increased representational generality improves the robustness of integration to fluctuations in arousal energy. 
Although increasing both depth and width increases the working range of energies relative to well depth, it is important to note the difference in how this occurs. While increasing depth increases the range of hypoarousal and incorporates higher energies into the working range, increasing width decreases the range of hypoarousal, but as a limited capacity to incorporate higher energies into the working range. Converse to increasing optimal arousal with increasing efficacy, increasing representational generality implies a commensurate decrease in optimal arousal as the ground state decreases.

Figure 9: Graph of eigenenergy across several widths (in a well of depth $d=5$ ). Note that, as well width increases, the eigenenergy associated with each eigennumber decreases. Note, also, that, as well width increases, the energy associated with the most excited eigenstate (highest eigennumber) necessarily does not excede a certain value, the depth of the well.

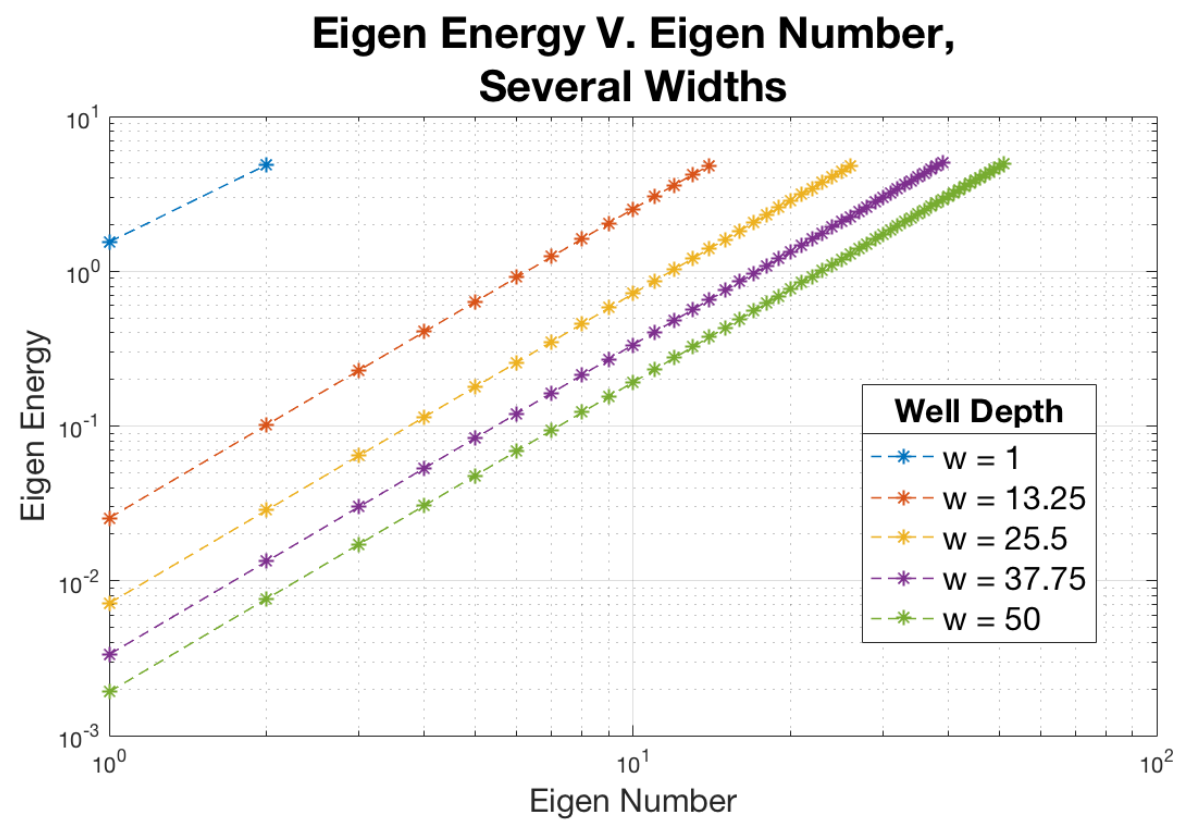

Increased Width May Motivate Periodic Categorical Stimulus Discretization As is the case for depth, and as shown by 11, the working range of energies increases generally as a function of width, while exhibiting nonmonotonicities corresponding to the appearance of new eigenstates. These non-monotonicities imply that performance benefits most from increases in representational generality that correspond to crossing a threshold at which 
a new eigenstate is introduced into the system, with "quantum leaps" in performance predicted at those widths.

Such an effect may manifest in the "natural" or "intuitive" categorization of continuous stimuli into categories of linearly increasing generality. For an illustration of this effect, we return to the example of color categorization: as an agent is tasked with sorting colors into a set of categories, the MPMW predicts that their performance will increase overall as categories increase in generality, exhibiting periodic discontinuities as categories cross thresholds of generality. Indeed, such discrete increases in representational generality are commonly used in teaching color theory, as the color wheel is commonly segmented first into three "primary" colors (red, yellow, blue), then into six primary and secondary colors (the colors of the rainbow), and finally primary, secondary, and tertiary colors (such as blue-green and indigo). There are other well-established color systems for addressing color theory in art, including seven-color color wheels (including the "ROYGBIV" system) [43] and trichromatic light-based (as opposed to pigment-based) color systems that form the basis for color displays, in particular cathode ray tube (CRT) displays [1], and "opponent process" light-based theory [25]. Among these, the trichromatic light-based color system was first postulated by physicists studying the visible spectrum of light and, considering how perception thereof must arise in humans, was proposed to be based on the three "principal" colors in pigment-based color theory, red, yellow, and blue [43]. The common thread among color theories is the capacity to reduce the infinite and continuous spectrum of visual color into principal colors, which could equally be termed "eigen colors", for the property that all color can be created by a linear combination thereof. Despite the common designation of red yellow and blue as the "primary" colors in pigment-based color theory, isolating the primary colors from naturally occurring pigments is not remotely a trivial process and the successful combination of red yellow and blue into a target color is highly sensitive to the source of the pigments, as any child trying to create their own color wheel with crayons or markers can attest. Thus, despite the commonality of the primary colors, their station as primary colors is not guaranteed by nature. Although we have focused on color here, similar effects occur in the categorization of pitch into octaves and then into linearly spaced steps between octaves, with the tension between linear and non-linear traversal of octaves used to create mood in music.

The predicted effect may be verified by an experimental paradigm in which an agent is tasked with sorting stimuli, randomly generated and uni- 
formly distributed across a field of continuous stimuli (such as color) as belonging or not belonging within a sub-field of that continuum. At each trial, the agent is presented with different categories, formed by pre-determined discretizations of the set at finely spaced intervals. The MPMW predicts that performance will increase overall with increased generality and will exhibit "quantum leaps" periodically. Furthermore, the quantum leaps may be smaller and more frequent for agents with greater efficacy, such as those more familiar with the stimulus type. An example of MIE curves v. stimulus generality at constant energy is shown in figure 10 .

\section{Hypoarousal May be Overcome by Increasing Representational} Generality Just as increasing efficacy results in increases in various performance parameters, given the correct range of arousal energy, so does increasing representational generality. In contrast to increasing efficacy, because increasing the generality of a stimulus decreases both the ranges of both hypo- and hyperarousal, if arousal is initially insufficient to perform a task, this can be overcome by a sufficient increase in generality. As with efficacy increases, increasing generality will initially produce erratic ("flickering") improvements in performance before these become stable. If arousal is initially too high to perform the task, performance will benefit from increased generality only in the case that arousal is within the threshold set by efficacy $\left(E<V_{\max }\right)$. Past this point, efficacy must also be increased to incorporate arousal into the working range of energies.

In summary, the MPMW predicts that, in a state of hypoarousal, performance can be improved by increasing generality and there is no lower bound on energies at which this effect disappears. However, as the decrease in hypoarousal is non-linear, this effect is asymptotic. Additionally, improvements in performance with generality will exhibit initial "flickering" discontinuities as the arousal range of the agent first reaches the working range of energies, followed by more stable improvements as generality further increases.

Conclusion: The Effect of Width on Performance In conclusion, the MPMW predicts several effects of linearly increasing representational generality. First, as with efficacy increases, increases in representational generality will increase the working range of energies, making processing more robust to arousal fluctuations. These increases will exhibit "quantum leaps" with constant periodicity (as opposed to the decreasing periodicity arising from 
Figure 10: MIE increases discontinuously as a function of width, with smaller and more frequent increases occurring for wells of greater depth.

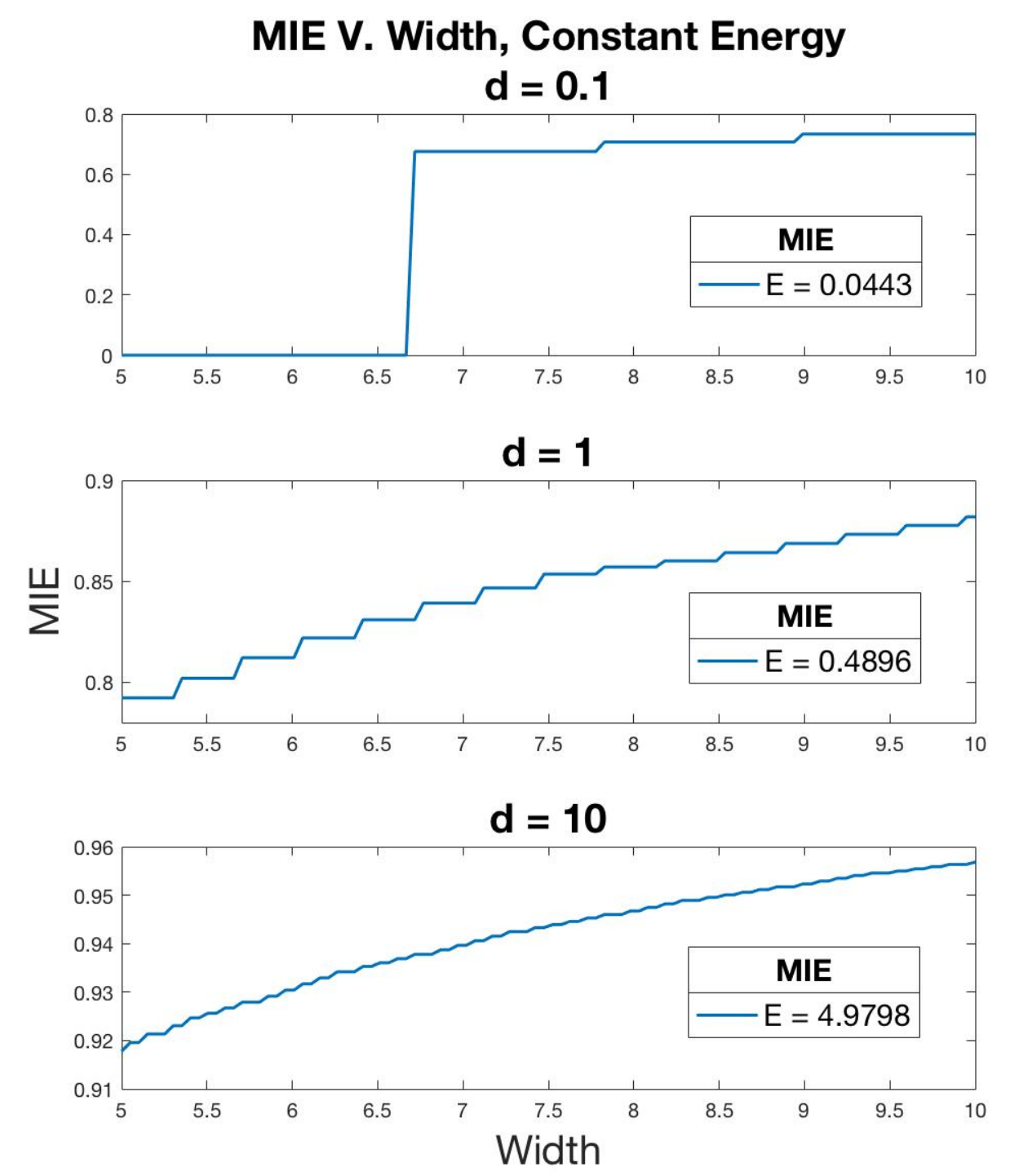


Figure 11: Top Left: Range of Hypoarousal V. Width at several depths. Log-Log scale. Range of hypoarousal decreases monotonically with width. Note that hypoarousal is always larger at larger depths, with the maximum range converging at very high depth.

Bottom Left: Range of Hyperarousal V. Width at several depths. Log-Linear scale. Range increases until a new eigenstate is introduced, then drops precipitously. These drops occur approximately linearly in width. Hyperarousal range is overall decreasing in width and increasing in depths, with range converging as depth increases. Top Right: Working Range V. Width at Several Depths. Working range increases approximately as a power law, with larger working ranges occurring at larger depths. Bottom Right: Fractional Working Range V. Width at several depths. Working range/depth increases approximately as a power law as width increases, saturating at 1. Larger working ranges/depth are associated with larger depths, despite the fact that the absolute ranges of hypo and hyperarousal both generally increase as a function of depth.
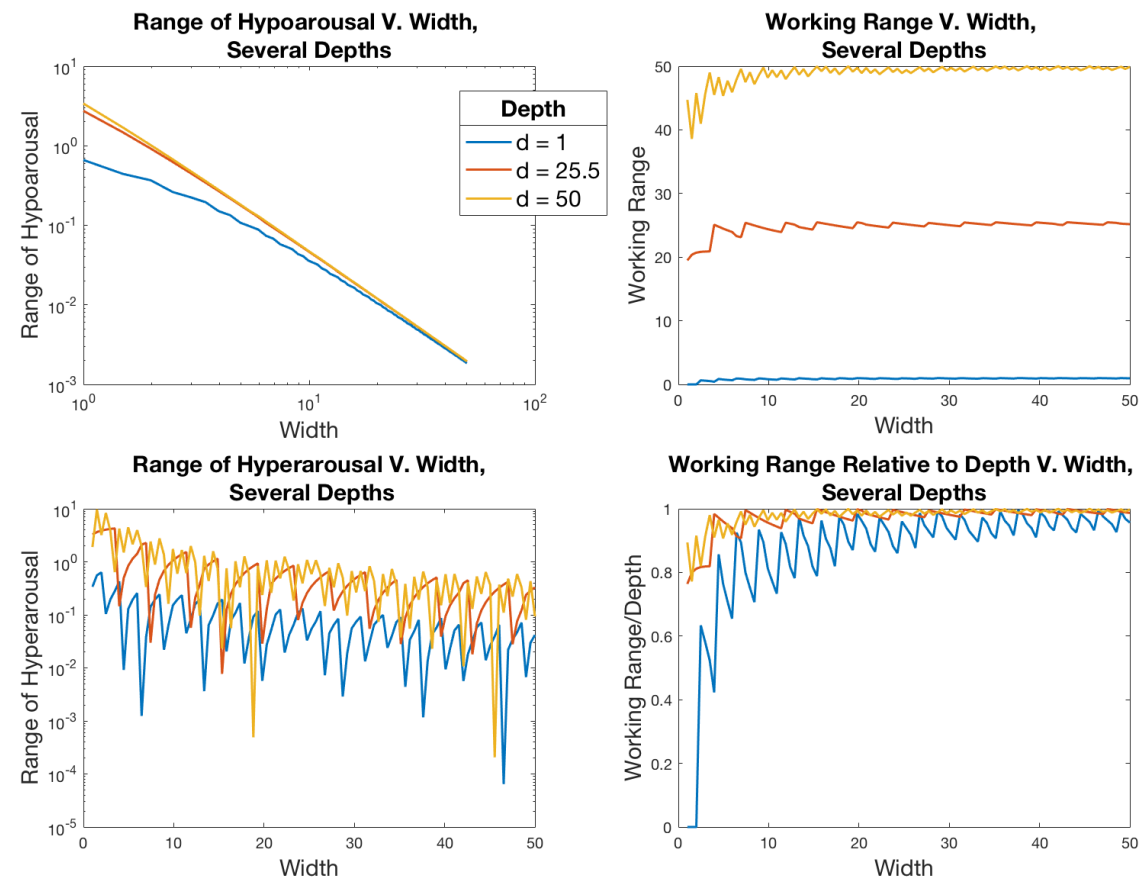
Figure 12: Mean MIE for an agent with constant arousal as stimulus generality increases. The top graph shows MIE for an agent initially in hypoarousal. The bottom graph shows the same for an agent initially in hyperarousal (bottom). Notice that both exhibit a "flickering" on. In the case of hypoarousal, agent with greater allocated efficacy exhibits less flickering and greater MIE, indicating that efficacy increases the efficacy of generality. This pattern does not hold of initial hyperarousal.
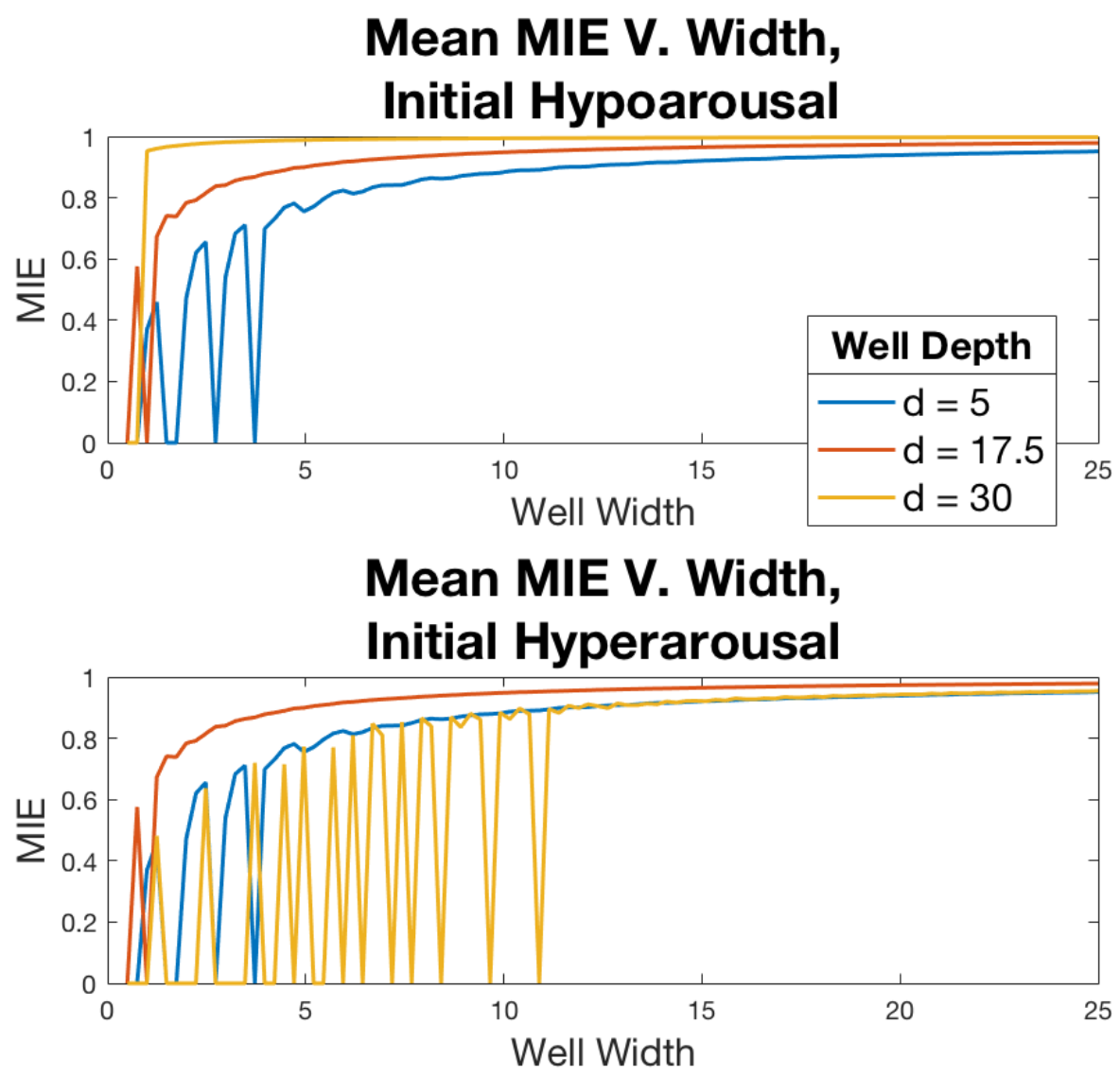

linear efficacy increases). Such "quantum leaps" may motivate the periodic discretization of continuous stimuli into linearly spaced categories, as occurs with the color wheel and pitch frequencies. As with efficacy increases, performance may be improved by increasing generality. However, where efficacy increases are best suited to overcoming performance detriments due to hyperarousal, generality increases are best suited to overcoming performance 
detriments due to hypoarousal.

\subsection{Conclusions: The Single Well Landscape}

In general, the MPMW makes similar but differentiable predictions about the effects of linearly increasing efficacy and representational generality in a single well system. Both will produce quantum leaps in performance parameters periodically, but increasing efficacy will produce these with decreasing frequency while increasing generality will produce them with constant frequency. These "quantum leaps" may motivate exponentially spaced discrete increases in allocated attention or in stimulus salience as agents seek improved performance on tasks by these means. Similar "quantum leaps" may motivate the clustering of continuous stimuli into linearly spaced discrete categories. Furthermore, where linearly increasing efficacy drives an increase in ground state energy that, in an optimal agent, will produce commensurately increasing arousal, linearly increasing generality will produce commensurately decreasing arousal. Finally, the MPMW predicts that agents will tend to increase efficacy to overcome performance detriments due to hyperarousal and increase generality to overcome performance detriments due to hypoarousal.

This section has established the effects of depth and width increases in a single well landscape, which is a special case of the types of landscapes the MPMW treats. The effects described here are generalizable to and provide an intuitive grounding for the effects of the same parameters in more complex landscapes. In the following section, we begin addressing these more complex landscapes with a discussion of double well landscapes.

\section{Double Well Landscapes}

\subsection{Introduction}

In a natural setting, an agent attempting to respond only to a target stimulus must filter out information from a wide variety of non-target stimuli. Representing this literally would require explicitly specifying wells for the target stimulus and all non-target stimuli, which is intractable. The single well landscape simplifies this by combining all non-target stimuli into the classically forbidden region. Doing so admits dropped bits as a source of 
error, producing slower responses or, with sufficiently many dropped bits, a non-response error. Introducing a second well to a landscape provides a more refined approach in which non-target stimuli that are task-relevant may be assigned to the second, "non-target" well (that we will refer to as "well 2 " by convention established in [48]). The non-target well is one for which either stimulus or response is "incorrect" at the time (incongruent with the response associated with the target stimulus). For our example pedestrian, foot traffic may be relegated to the non-target well, as it may contain information about whether crossing is safe, but is not the stimulus upon which the pedestrian wants to pin their response. Meanwhile, all task-irrelevant stimuli are relegated to the background. In addition to dropped bits, such a landscape also admits bits measured to occupy well 2 as an additional source of error. The latter, like dropped bits, can slow response time for correct responses or, in sufficient number, produce an erroneous response (i.e., the one associated with well 2).

Importantly, in some settings, including many laboratory paradigms, stimuli that are designated as, or assumed to be, task-irrelevant may nevertheless carry information relevant to determining the task-relevant response. This can occur if such irrelevant stimuli either consistently favor the taskrelevant response (often referred to as "congruent" stimuli), or consistently favor the task-irrelevant response ("incongruent" stimuli"), both of which can be relevant to the allocation of controlled attention (and thus the determination of well depth): If congruent stimuli are prevalent, then attending to them can help improve task performance by providing an additional source of evidence in favor of the correct response. Conversely, if incongruent stimuli are prevalent, suppressing them can also improve performance. Such effects have been observed in laboratory studies [30], [23], [14], and addressed using classical models that treat these circumstances as a 2AFC task [7].

In [48] we analyzed the classical component of a two-well model within the MPMW framework. Here, we extend the analysis of that model, to address the effects of well parameters and their interaction with arousal. We begin by first considering the symmetric double square well, which most closely relates to the single square well landscape discussed above. We then consider the more general double well landscape by examining the effects of deviations from symmetry. 


\subsection{Symmetric Double Well Landscape}

The simplest double square well landscape is one in which the wells are of equal depth and width with a finite separation between them. As noted in [49] and elsewhere, such symmetric systems exhibit eigenstates the energies of which appear in closely spaced or identical pairs and with nearly the same number of energies as those of a single square well of equal depth and width given by $w=w_{1}+w_{2}$. Additionally, when plotting the eigen energies of a symmetric square well system against their eigennumbers, the curve for the double square well intersects the curve for the single square well of width $w=w_{1}+w_{2}$ between each of the paired energies; that is, the eigen energies of a symmetric double square well system are not identical to, but are similarly distributed to those of the corresponding single square well system. Each eigenstate pair corresponds to one symmetric and one anti-symmetric state, which have nearly identical PDFs, and all of which describe the particle as equally distributed across both wells and therefore having equal probability of being found in each, $M_{1, j}=M_{2, j}$.

Combined States of Double Well Landscape In a symmetric double well, combined states, such that

$$
E=a E_{j}+(1-a) E_{j+1}
$$

will exhibit MIEs that are equal for both states on average, but that oscillate in time as described in section 3.2.1 above. As the sate oscillates, so do the MIEs, producing effects such as drift rate reversal over time. With the correct linear combination of eigenstates, a combined state can be constructed that is, at one point in time, completely localized to one well and, at a later point, completely localized to the other. As also noted in section 3.2.1, observing such an oscillation would require the repeated sampling of a single particle, as is done in other quantum cognitive models. In future work, a single-particle multi-well model may be constructed to exploit this feature of combined states to formulate models of bistable or multistable perception.

Barriers The double well landscape includes a parameter that does not exist in the single well landscape: the barrier separating the wells. The barrier's potential is, by definition, equal to the background potential $\left(V_{\text {barrier }}=\right.$ $\left.V_{\max }\right)$, as it would otherwise be considered a third well with zero separation 
Figure 13: Top Left: Range of Hypoarousal V. Width for a double well landscape at several depths. Log-linear scale. Range of hypoarousal is overall decreasing in width and larger at higher depths. Nonmonotonicities are attributable to the introduction of new eigensates and are most pronounced in narrower wells. Bottom Left: Range of Hyperarousal V. Width for a double well landscape at several depths, log-linear scale. Range increases until a new eigenstate is introduced, then drops precipitously. These drops occur approximately as the square root of depth. Hyperarousal range is overall decreasing in width. Top Right: Working Range V. Width for a double well landscape at Several depths. Working range increases sublinearly with large increases associated with new eigenstates. Bottom Right: Fractional Working Range V. Width for a double well landscape at several depths. Working range/depth increases as width increases, saturating at 1 .
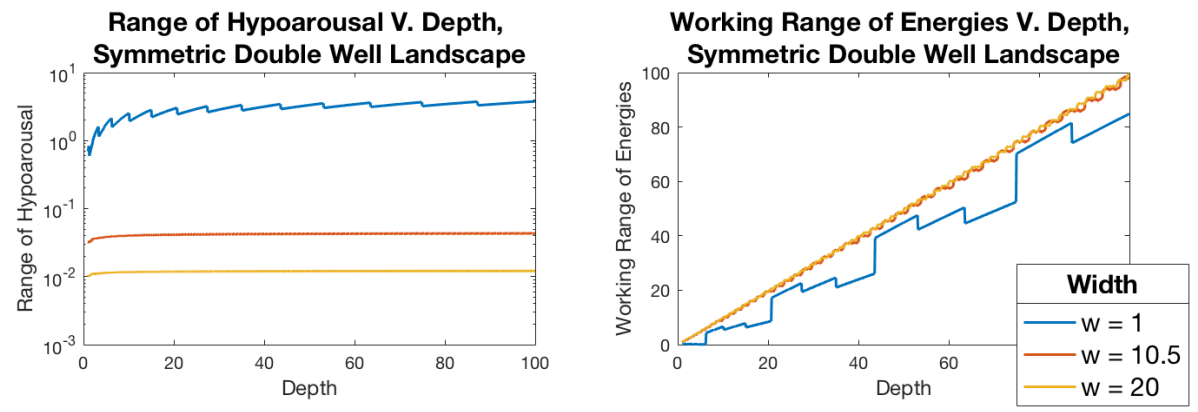

Range of Hyperarousal V. Depth,

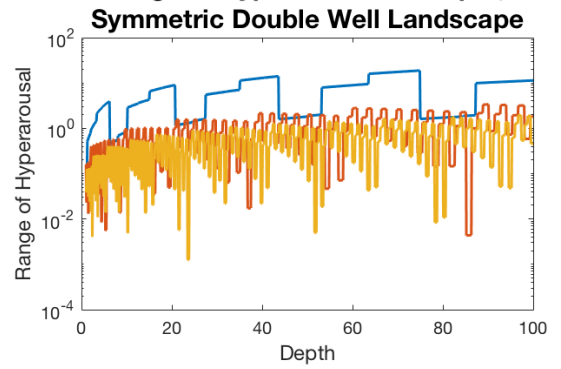

Fractional Working Range of Energies V. Depth, Symmetric Double Well Landscape

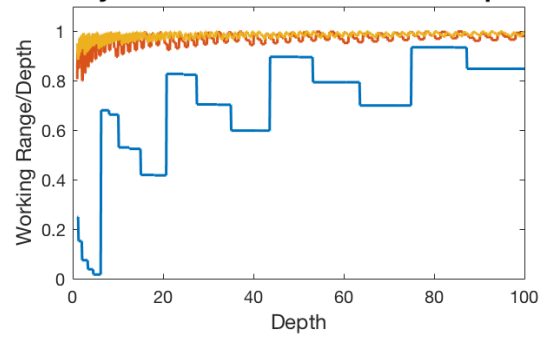


Figure 14: Progressive localization of wave function to wider well as width asymmetry increases. Notice that nearly complete localization occurs at very low asymmetry, and that localization occurs discontinuously.

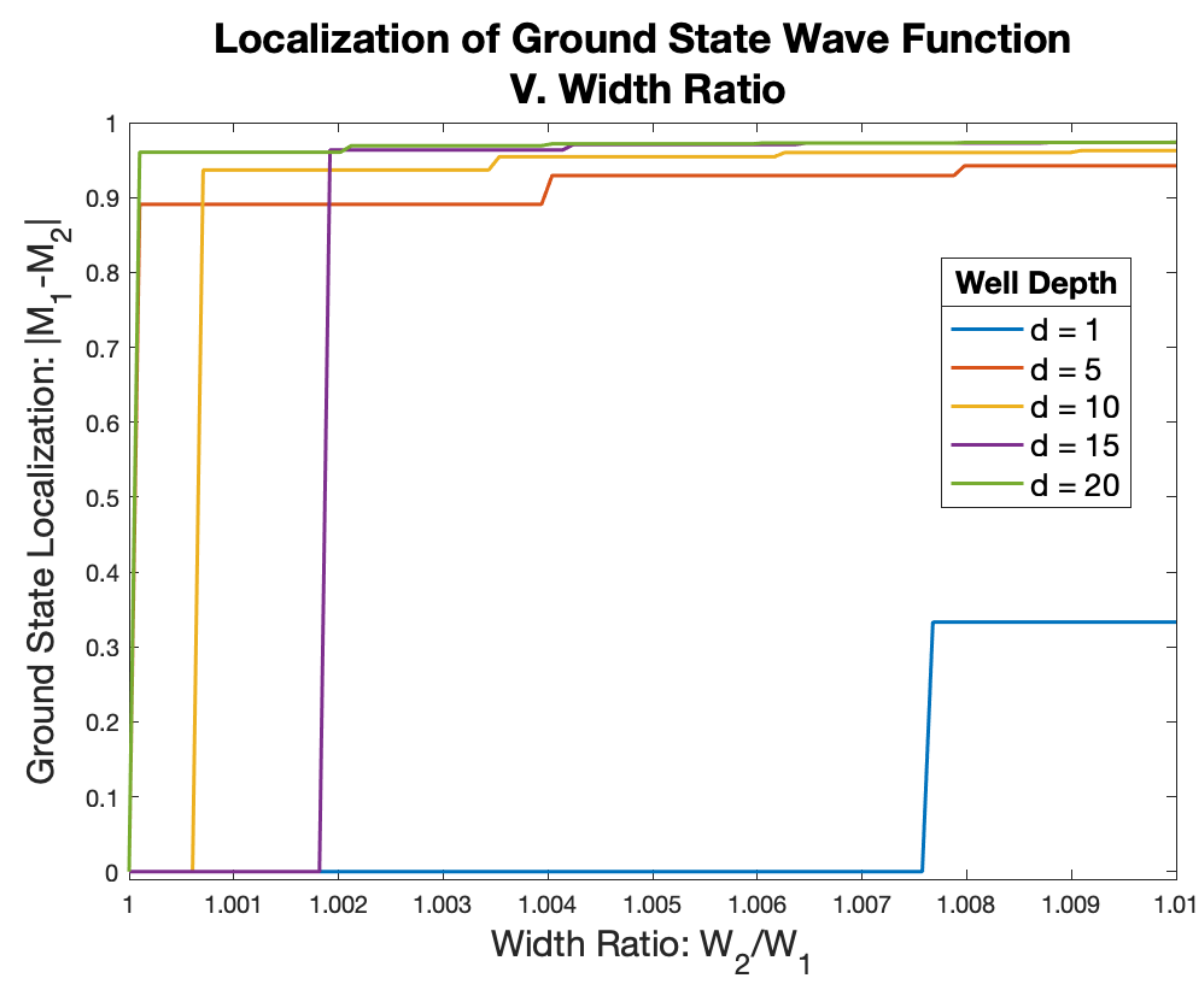


between adjacent wells. The width of the barrier represents the semantic or representational separation of task-relevant stimuli corresponding to the two responses. This mapping implies that, as stimulus-response mappings grow more similar to one another and the state function (and PDF) has less space to decay between the wells, the components of the state exhibit greater interference with one another.

Figure 15 illustrates this effect: For a very narrow barrier, well-associated components of the state are closely joined and there is little space in which the eigenstate can decay between the two wells. This increases the probability that a particle will be found inside the barrier, thereby reducing the MIEs of the wells. As barrier width increases, the eigenstate decays more toward its center, and the components of the eigenstate in each well decouple from one another. This increases the MIEs for each well by localizing the particle to the wells. This decoupling also occurs in asymmetric double well landscapes, as discussed further on.

Deviations from Symmetry Produce Localized States Previous work has shown that states evenly distributed in symmetric double-well landscapes become heavily localized in landscapes with depths that deviate only very slightly from symmetry [18]). As illustrated in figure 14, this is also true for systems with widths deviating slightly from symmetry. For both cases, even extremely small deviations from symmetry will result in an uneven distribution of states between wells $\left(M_{1} \neq M_{2} \neq 0\right)$, and slightly larger asymmetries produce heavily localized states $\left(M_{1} \gg M_{2} \approx 0\right)$ with adjacent energy states localized to different wells $\left(M_{1, j} \gg M_{2, j} \approx 0 ; M_{2, j+1} \gg M_{1, j+1} \approx 0\right)$. We refer to states as localized to one well when the component of the state in that well exceeds the component of the state in any other well.

As asymmetries grow, progressively larger bands of energy, clusters of eigenstates, will be localized to only one well. In future sections, we refer to the width of a band of energies within which states are localized to the same well "bandwidth". This effect can be understood by considering how the eigenstates of each well, in its own separate landscape, would change. A widening or deepening well, in isolation, would gain new eigenstates in a pattern determined by the parameter that is increasing. Similar effects are present as asymmetries increase in double well systems, but eigenstates, while primarily localized to one well, will have some non-zero component present in the other. 
Figure 15: Example PDF for a double well landscape. Note that closer wells have more closely coupled PDFs and therefore exhibit greater interference in the form of a higher probability of dropping a bit.
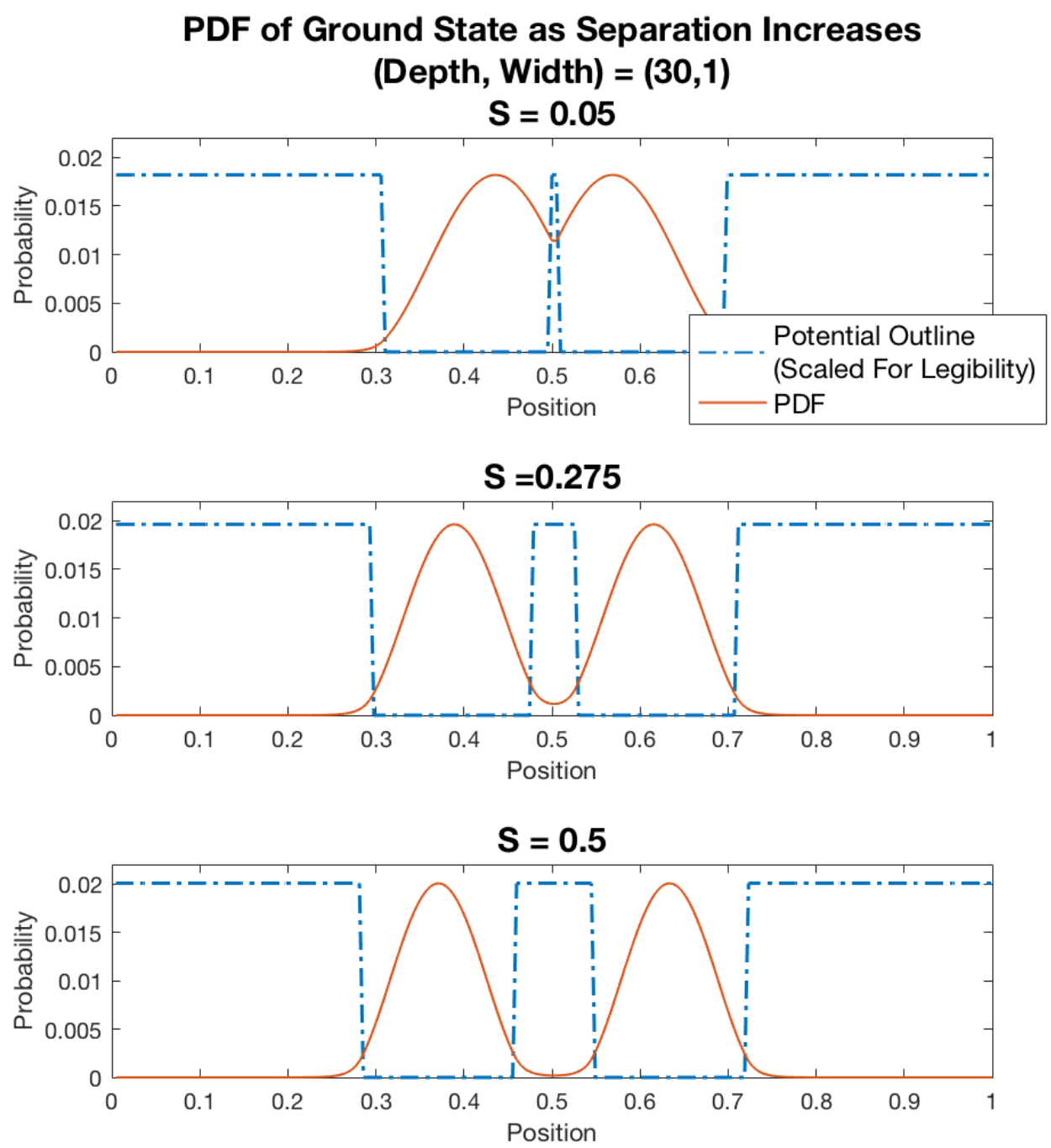
As in the case of a single well landscape, the MPMW makes differentiable predictions about the effects of depth and width both in symmetric and asymmetric landscapes. In the remainder of this section, we will address the effects of each in turn.

\subsection{Effects of Depth on Eigenstates}

Increasing Depth Symmetrically In many ways, the double square well system, especially in the symmetric case, parallels a single square well system. In addition to access to the classically forbidden region and the general forms of the eigenstates, higher eigen numbers generally correspond to more loosely bound states and therefore smaller MIEs. Finally, there also exist regions of both hypo- and hyperarousal. Figure 13 shows that the relationships between depth and the ranges of hypo- and hyperarousal in the symmetric double well landscape are qualitatively identical to those in the single well landscape. Increasing depth increases both the range of hypoarousal and the working range of energies, with quantum leaps occurring when new eigenstates become available.

Large Asymmetries in Depth Bifurcate the Working Range of Energies As mentioned above, deviations from symmetry in depth produce localized states. At minimal asymmetry, adjacent states are localized to opposite wells, as illustrated in figure 18. Because states are so closely spaced in minimally asymmetric landscapes, tiny fluctuations of arousal produce reversals in drift rate, resulting in longer response times and larger numbers of errors.

However, as depth asymmetry increases, the working range of energies can be differentiated into two sub-ranges. The lower range of energies, those that are greater than the ground state of the deeper well but less than the ground state of the shallower well (the lowest energy eigenstate localized to the shallower well) and the upper range of energies for which $V_{2} \approx<E<V_{\max }$. As depth asymmetry increases, so does the lower range of energies, resulting in more robust processing of the stimulus associated with the deeper well when arousal occupies that lower range of energies. Because higher energy states are more "loosely bound", increases in arousal that fall within the lower range of energies will still increase the probability of "dropping a bit", increasing response times, but not reversing the decision variable's trajectory. 
Figure 16: Top Left: Range of Hypoarousal V. Depth for a double well landscape at several widths. Log-linear scale. Range of hypoarousal is overall increasing in depth and larger at narrower widths. Nonmonotonicities are attributable to the introduction of new eigensates and are most pronounced in narrower wells. Bottom Left: Range of Hyperarousal V. Depth for a double well landscape at several widths, log-linear scale. Range increases until a new eigenstate is introduced, then drops precipitously. These drops occur approximately as the square root of depth. Hyperarousal range is overall increasing in depth and decreasing in width, with range converging as width increases. Top Right: Working Range V. Depth for a double well landscape at Several widths. Working range increases approximately linearly with large increases associated with new eigenstates. At very large width, working range converges to depth, causing it to increase linearly with smaller stepwise effects. Bottom Right: Fractional Working Range V. Depth for a double well landscape at several widths. Working range/depth increases as depth increases, saturating at 1 . That is, despite the growth of the ranges of hypo and hyperarousal, growth of depth outpaces them. Larger working ranges/depth are associated with larger width.
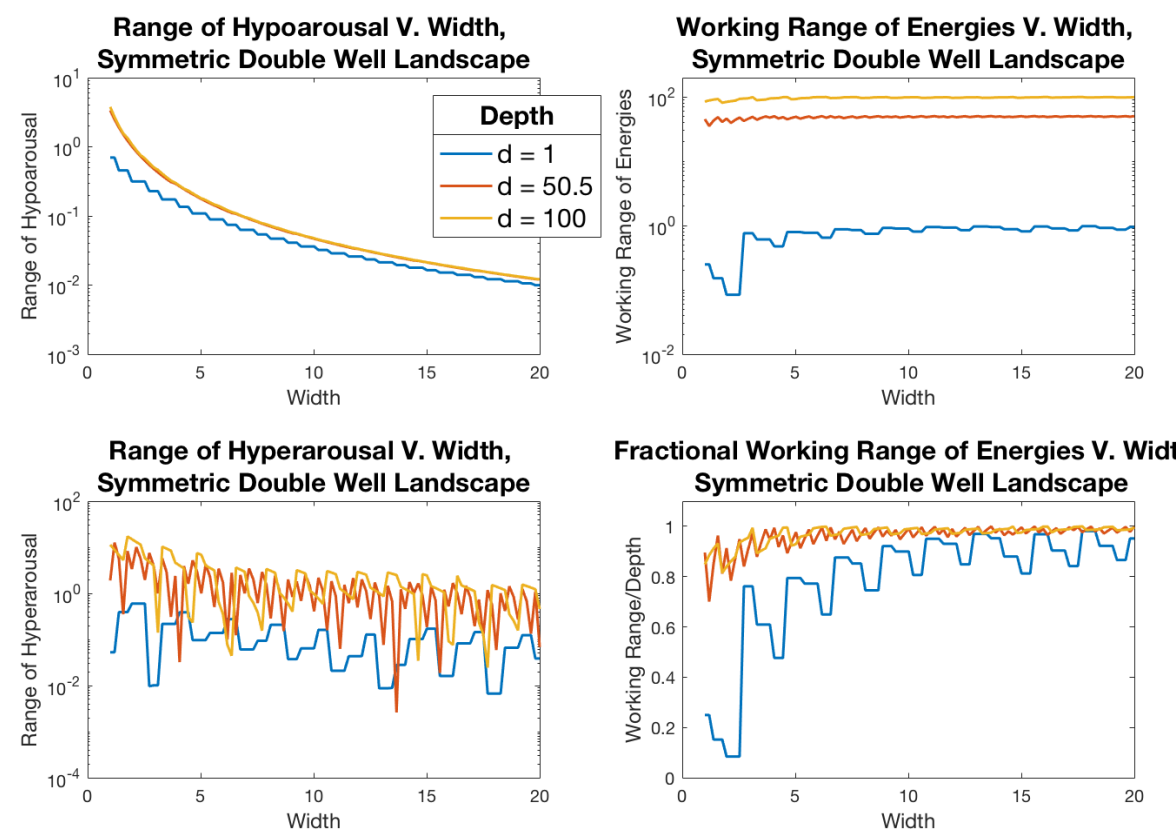
In addition to expanding the lower working range of energies, increasing depth asymmetries effect both magnitude (MIEs and drift rates) and robustness (bandwidth and variance) of processing biases in both the lower and upper range of working energies. At arousal within the lower range of energies, the asymmetrically allocated efficacy biases processing in favor of the target (deeper) well, resulting in more efficient integration of information relevant to that stimulus (i.e., high MIE and drift rate, few bits integrated incorrectly or dropped). As arousal increases to the cusp of the lower range of energies, performance will deteriorate abruptly, as it enters a transitional band of energies in which drift rate is low and variance is high. In the upper range of energies, the two stimuli will exhibit conflict, with alternating bands of energies localized to each well. This conflict causes MIEs for the deeper well and drift rate in the upper range of energies to be lower than in the lower range, an effect that grows more pronounced as depth asymmetries increase.

To formally verify the effects of increasing depth asymmetry on the magnitudes of MIEs and drift rates, we calculated the mean drift rate for both the lower and upper ranges of energies for five landscapes with widths ranging from 5 to 30 and with initially equal depths $\left(d_{0}=5\right)$ as the target well's depth increased to twice the depth of the non-target well. We then calculated the mean of the drift rates at each depth across the five width sets. Using non-linear least squares, we found that the resultant curve fit to an increasing power law of the for $M=a{\frac{V_{1}}{V_{2}}}^{b}+c$ with an $R^{2}=0.9325$. We performed the same calculation and fit for the drift rates in the upper region of energies, and found that the resultant curve fit a decreasing power law of the same form with an $R^{2}=0.9779$. These curves and their fits are illustrated in figure 17 .

It is trivial to state that processing in the lower range of energies grows more robust to arousal perturbations as depth asymmetries increase. However, non-trivially, the upper range of energies exhibits the reverse of this effect, with wider bands of energies localized to the shallower well, so that integration overall favors the shallower well (non-target stimulus) as illustrated in figure 18. As depth asymmetries increase, these bands widen and processing bias in favor of the non-target stimulus becomes progressively more robust. To formally demonstrate this point, we calculated the mean bandwidths of energies localized to the shallower well in the upper range of energies for five sets of landscapes. Each landscape began symmetric, with widths ranging from 5 to 30 . For each landscape, we calculated the mean 
Figure 17: Mean drift rates for the lower (top graph) range of energies and upper (bottom graph) range of energies as depth asymmetries increase. Note that, while integration in the lower range is progressively dominated by the target well, integration in the upper range is progressively dominated by the non-target well, so if an agent's arousal shifts sufficiently as to push them into the upper range of energies, they will likely experience a drift rate reversal as the non-target stimulus takes over integration.
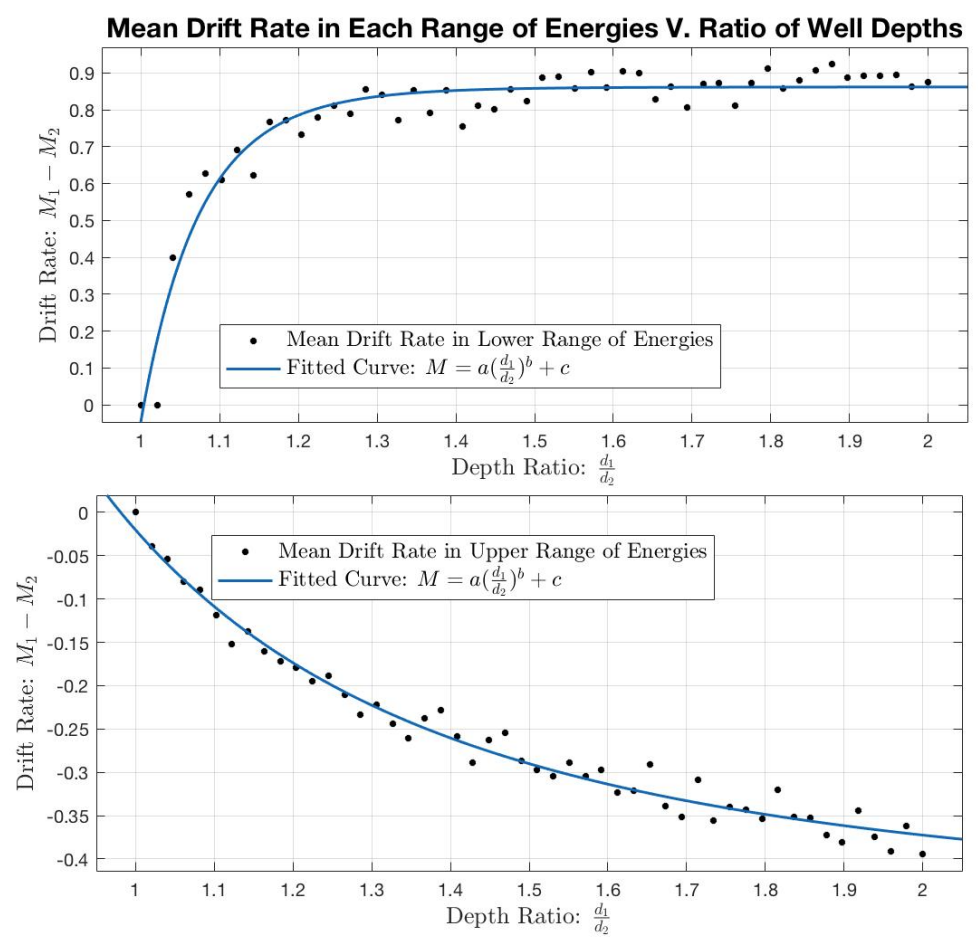
bandwidth at each of 50 depth asymmetries as the target well increased to twice the depth of the non-target well. We found that, for all landscapes, the mean derivative of the bandwidth as a function of depth asymmetry $\left(\frac{\partial b w)}{\partial(d 1 / d 2)}\right.$ was positive, without a clear trend in the value of the mean as a function of width. This shows that increasing depth asymmetry, in general, increases the robustness of processing biases to perturbations in arousal.

Figure 18: MIE V. Arousal in three width-symmetric, depth-asymmetric sample landscapes. Top: Nearly symmetriclandscapes. Well 1 and well 2 have high conflict, with each dominating a narrow bands of energy and neraly equal MIEs. Middle: Large asymmetry $\left(d_{1}=2 d_{2}\right)$. Well 1 dominates the lower range of energies with a small section of transitional energies where arousal approaches $V_{2}$. Well 2 begins to "crowd out" well 1 in the upper range of energies. Bottom: More exaggerated depth asymmetry $\left(d_{1}=3 d_{2}\right)$. Patterns from previous graph are more pronounced, with well 2 more robustly dominating processing in the upper range of energies.
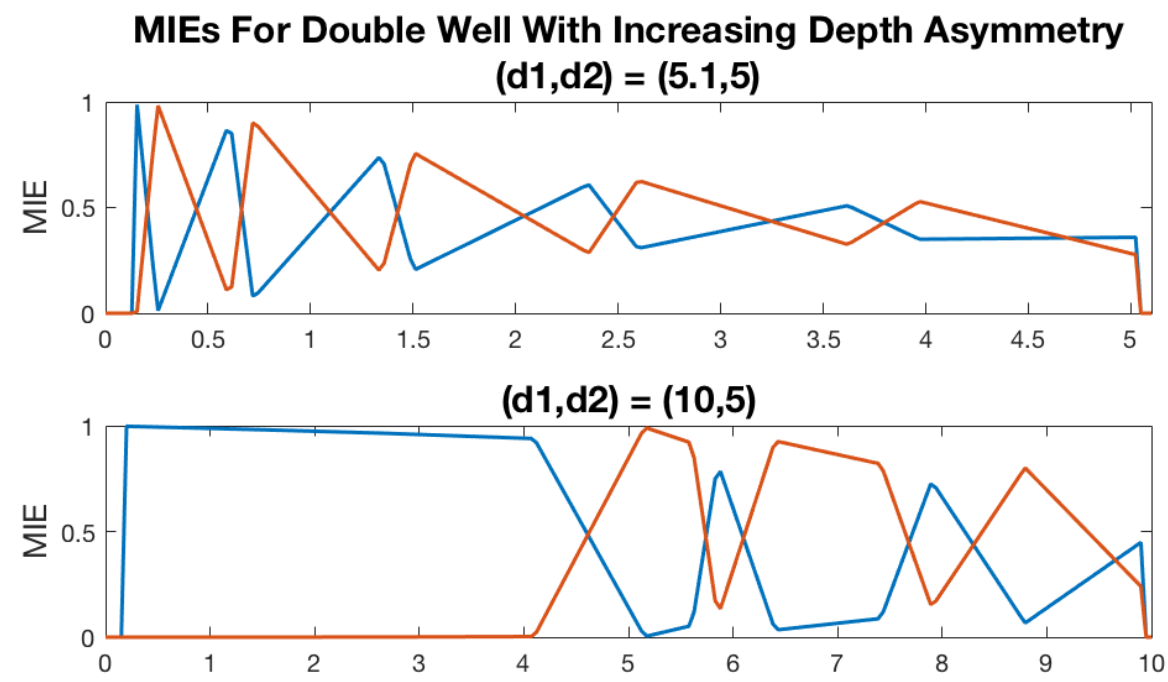

$(d 1, d 2)=(15,5)$

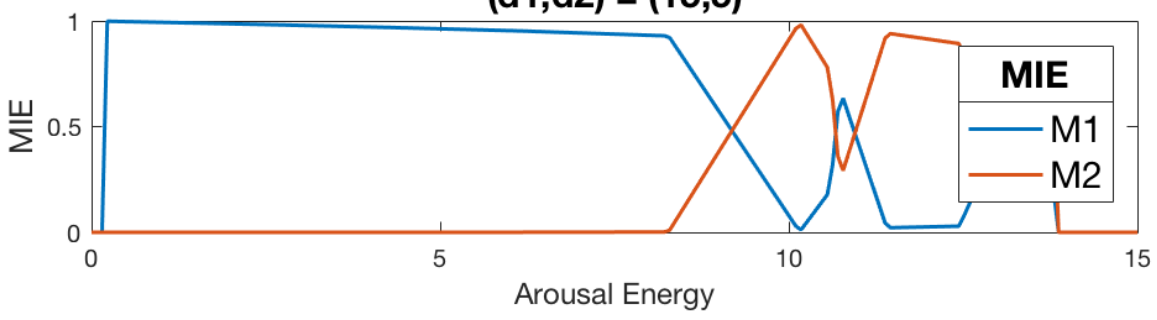


Depth Asymmetries are More Impactful for Wider Wells As we will discuss briefly, and just as in single well landscapes, wider wells in a double well landscape are associated with a greater number of eigenstates across the entirety of their depth. Therefore, it is intuitive that differential efficacy allocation should be more impactful in the lower range of energies for a higher generality pair of stimuli than for a lower generality one. However, this increased impact of differential efficacy may be difficult to detect at small width differences. Therefore, to test for this effect, we calculated the mean drift rates across the upper and lower ranges of energies for a set of five landscapes of different widths increasing exponentially from 5 to 125 as one well increased to twice the depth of the other well, with drift rate calculated at 50 depth asymmetries. We then calculated the differences between drift rates at each depth for each width and the next smallest width. We found that both the mean and median differences in these sets were positive for $100 \%$ of sets, with the mean and median differences in drift rate decreasing as differences in width increased. The absolute mean difference in drift rates across all depth asymmetries and widths was 0.0489 , and the mean of the median difference was 0.0307 . The mean and median differences in drift rate are plotted against the difference in widths in figure 19.

In the upper range of energies, the effects of larger symmetric widths on the decrease in drift rate as depth asymmetry increases is less clear. Performing the same analysis as on the lower range of energies, we found that three of four of each mean and median differences were negative. This indicates a relative decrease in drift rate in the upper range of energies as well width symmetrically increased. However, there was not a clear functional trend in how width affected these differences.

Depth Asymmetries Reproduce Familiar Stability-Flexibility Tradeoffs The effects described above can be used to make predictions about how depth asymmetries in the MPMW give rise to familiar stability-flexibility tradeoffs described in the cognitive science literature [56], [22]. Stabilityflexiblity tradeoffs are phenomena in which an agent must choose between higher and more robust performance and the ability to rapidly adjust performance to address changing needs.

The first stability-flexibility tradeoff the MPMW predicts is associated with task switching and requires balancing robust, efficient integration with the time cost of efficacy reallocation to meet changing task demands. As- 
Figure 19: Mean and median differences in drift rate in the lower range of energies of landscapes of different widths. Note that differences are positive, indicating that increasing with increases the efficacy of allocated efficacy. Note also that differences are decreasing, indicating that the positive impact of generality on the efficacy of efficacy is diminishing as a function of generality.

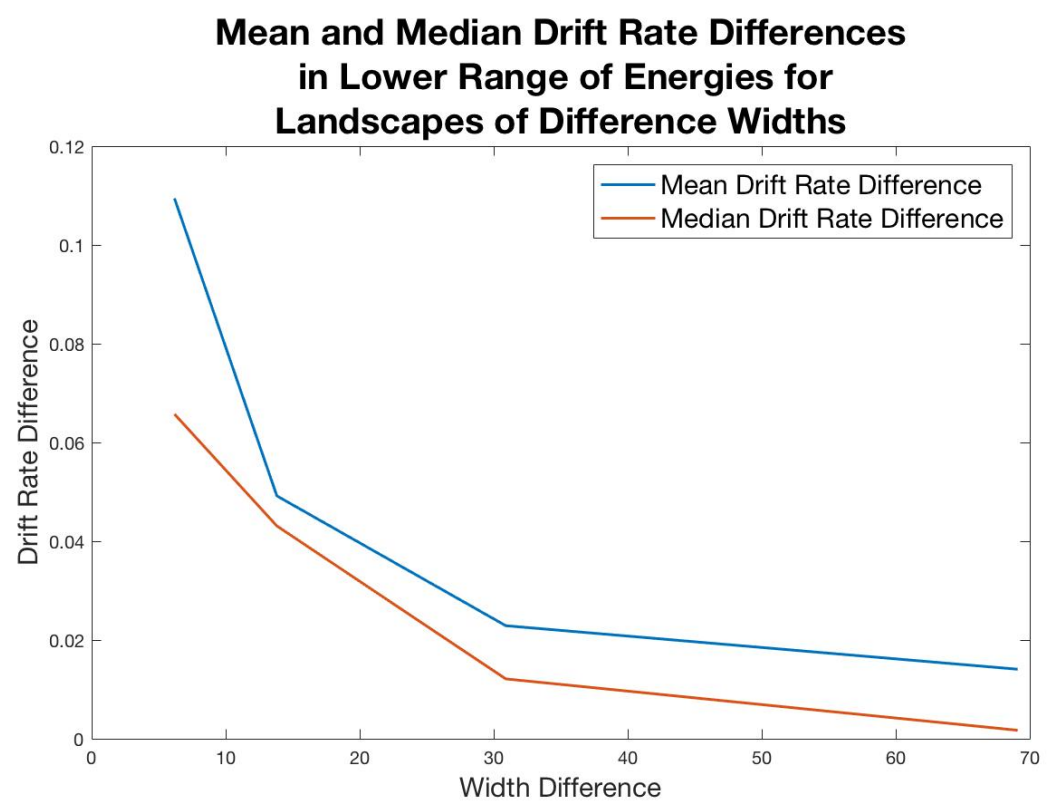


suming that finite efficacy recruitment requires finite time, an agent wishing to reallocate efficacy to bias processing toward a different stimulus will need more time to reverse a large asymmetry than a small one. A large efficacy asymmetry implies higher performance with a higher switch time. This tradeoff replicates one that has been treated in other models of decision-making and task-switching dynamics, and serves as a basic point of validation for the MPMW (see [39], [40]).

The second stability-flexibility tradeoff predicted by the MPMW is associated with adaptive responses to fluctuations in arousal. As demonstrated above, preferentially allocating efficacy to one stimulus without fully withdrawing it from another (which would reduce the system to a single well system) produces integration biases that are sensitive to arousal fluctuations. The extent to which arousal fluctuations impact MIE depends on efficacy asymmetry. Furthermore, the extent to which different depth asymmetries reduce sensitivity to non-target stimuli offers an array of solutions to the arousal-based stability-flexibility tradeoff, each of which may be optimal under different circumstances. That is, greater efficacy asymmetry further reduces sensitivity to non-target stimuli that may contain important information, despite potentially meaningful arousal fluctuations. Agents wishing to sporadically sample non-target task-relevant stimuli (e.g., the pedestrian sampling both the crosswalk signal and foot traffic) may benefit from smaller depth asymmetries that allow them to do so through small, potentially spontaneous, fluctuations in arousal. Such a landscape configuration will produce slower responses to either stimulus when they are incongruent (offer conflicting information about the correct response), but fast responses when they are congruent. Agents wishing to sustain focus on only one stimulus for a lengthy period of time despite information from other stimuli (e.g., a student studying in a noisy classroom) may benefit from a large depth asymmetry to render efficient, robust processing for their target stimulus.

Finally, two common behavioral phenomena that arise from the inability or failure to sufficiently address the arousal-based stability-flexibility tradeoff. The first we will term "hyperfocus" (borrowed from ADHD literature such as, [3], [15], [35]), in which an agent inadvertently ignores important stimuli (the bell ringing to signal the end of class) in favor of a target stimulus (their studies) due to an excessive depth asymmetry. The converse, that we term "hypofocus", occurs when depth asymmetry is insufficient and an agent is incapable of making decisions or completing tasks due to the resultantly high conflict between competing stimuli or tasks. Both of these 
phenomena manifest as symptoms of ADHD and other neuropsychological disorders. That they, among other common stability-flexibility phenomena are emergent properties of the MPMW serves to further validate it as a formal framework that encapsulates a rich variety of phenomena from across multiple disciplines.

Summary of the Effects of Depth Asymmetries In summary, we find several results concerning the impact of depth asymmetry on performance for a width-symmetric double well landscape. First, large asymmetries in depth bifurcate the working range of energies into a lower and upper component. In the lower range of energies, states are localized to the deeper (target) well, while the opposite is true in the upper range.

As the lower range of energies increases, so does the average drift rate associated with it, favoring the target stimulus. This effect increases with well width, such that the same depth asymmetries produce larger effects on drift rate in a wider pair of wells than in a narrower pair. This denotes that the differential allocation of efficacy is more impactful when applied to more general stimuli. However, the increase in efficacy impact diminishes with width. That is, as width increases, the increase in efficacy impact per unit width decreases and impact saturates at large widths.

In the upper range of energies, depth asymmetry has the opposite effect as in the lower range, biasing processing in favor of the shallower (non-target) well as asymmetries in depth increase. This denotes that differential allocation of efficacy to a target well is helpful only where arousal remains within the lower range of energies and may be counterproductive otherwise. Therefore, there is a contextually optimal level of differential efficacy allocation such that arousal remains within the lower range of energies throughout the duration of the task.

Where asymmetric efficacy favors the target stimulus, processing is optimal at the ground state. Increasing arousal from the ground state of the system will first produce a larger number of dropped bits within the lower range of energies, then to a large number of bits integrated in favor of the non-target stimulus in the upper range, and finally to a state of hyperarousal. These effects will manifest as slowing response times, then increased erroneous responses and, finally, a large number of non-response errors, respectively. In aggregate, the effects of depth asymmetry produce an "all or nothing" effect in which, as asymmetry increases, performance improves in the lower range 
of energies and deteriorates in the upper range.

These results are consistent with, and anticipated by, the patterns of eigenstates and MIEs observed in a single well system: deeper wells contain a larger number of eigenstates that cluster near the ground state, and thus have higher MIEs. Considering each well in a width-symmetric, depth-asymmetric double well landscape independently, we can anticipate that, as the deeper well increases in depth, a greater number of its higher perfoming eigenstates will sit below the ground state of the shallower well. Meanwhile, those states most localized to the shallower well will tend to cluster near its ground state, occupying energies between those energies localized to the shallower well.

\subsection{Effects of Width on Eigenstates}

Increasing Width Symmetrically Figure 16 shows that the relationships between width and the ranges of hypo- and hyperarousal in the symmetric double well landscape are qualitatively identical to those in the single well landscape. As in the case of the single finite well, increasing width decreases the ranges of hypo- and hyperarousal, but will not allow an agent to overcome hyperarousal associated with scattering states $\left(E>V_{\max }\right)$. Therefore, just as in the case of a single finite well, increasing representational generality is well-suited to overcoming hypoarousal and is limited in its ability to overcome hyperarousal. Additionally, as in the single well case, increasing width linearly produces quantum leaps in the working range, occurring when new eigenstates become available.

Deviations from Symmetry: Width As illustrated in figure 20, asymmetries in width produce alternating bands of energies in which states are localized to alternating wells. Drift rate magnitude $\left(\left|M_{1}-M_{2}\right|[48]\right)$, is highest and variance lowest near the center of each of these bands (skewed toward lower energies), with greater conflict occurring at their edges. Arousal fluctuations sufficient to traverse these bands produce reversals in drift rate direction, resulting in longer response times, larger numbers of non-responses, and/or lower accuracy, depending on decision thresholds (see [48]).

As width asymmetry increases, new eigenstates localized to the wider well are introduced to the system, and the bands of energies localized to the wider well grow in width," crowding out" those localized to the narrower well. Simultaneously, drift rate magnitude in the bands localized to the wider well 
increase. This means processing bias toward the more representationally general stimulus becomes both more efficient and more robust to perturbations in arousal as that generality increases. That is, arousal fluctuations of the same magnitude have a smaller effect on drift rate magnitude and produce fewer drift rate reversals as the generality of the target stimulus increases relative to that of the non-target stimulus.

To understand this effect intuitively, consider a color categorization task in which the color wheel is split into two equal components and each stimulus must be categorized as being more similar to one or the other. With some variation among individuals, this produces a symmetric double well landscape. However if, over time, one category begins to grow, occupying a broader band of colors, the landscape becomes asymmetric and, given a stimulus that falls precisely between the two categories (so that both wells are of equal depth), the probability of sorting the color into the more general category will become progressively higher as that category expands, with decreasing variance in responses as a result of changing bandwidths.

To verify these effects formally, we calculated mean drift rate and mean bandwidth across a set of twenty-five initially symmetric landscapes, initialized at five different depths and five different widths, as the width of one well increased to twice the width of the other well. We assigned the wider well to be the target well, with MIE $M_{1}$, so that drift rate $\left(M_{1}-M_{2}\right.$ [48]) was positive where the wider well dominated integration. For each of these landscapes, the mean drift rate generally increased with width asymmetry. Both mean and median derivatives of drift rate in width asymmetry were positive for all landscapes. This was accompanied by broadening bands within which the target well dominated integration. We found that the mean and median derivatives of bandwidth with width asymmetry were positive across all landscapes. These effects are visually represented for several sample landscapes by figure 20 .

In summary, the MPMW predicts that two equally representationally general stimuli, if equally efficacious, will compete equally for processing, resulting in a mean drift rate of zero and high variance. When two stimuli are equally efficacious, but one stimulus is more representationally general than the other, processing will be biased in favor of the more general stimulus, with increasing generality asymmetry resulting in increasing drift rate in favor of that stimulus. The MPMW also predicts increasingly robust processing biases with increasing width asymmetry. This results in higher drift rate and lower drift rate variance as the generality of the two stimuli become less 
Figure 20: MIEs V. arousal for three sample landscapes of symmetric depth $(\mathrm{d}=5)$ and asymmetric width. Top: barely asymmetric widths, energy bands in which $M_{1}>M_{2}$ and vice versa are approximately equal in width and MIEs are similar in magnitude. Middle: increasing width asymmetry $\left(w_{1}=2 w_{2}\right)$. Bands dominated by well 1 are significantly broader than those dominated by well 2. MIEs in bands dominated by well 1 are significantly larger than those in bands dominated by well 2. Bottom: very large width asymmetry $\left(w_{1}=3 w_{2}\right)$.
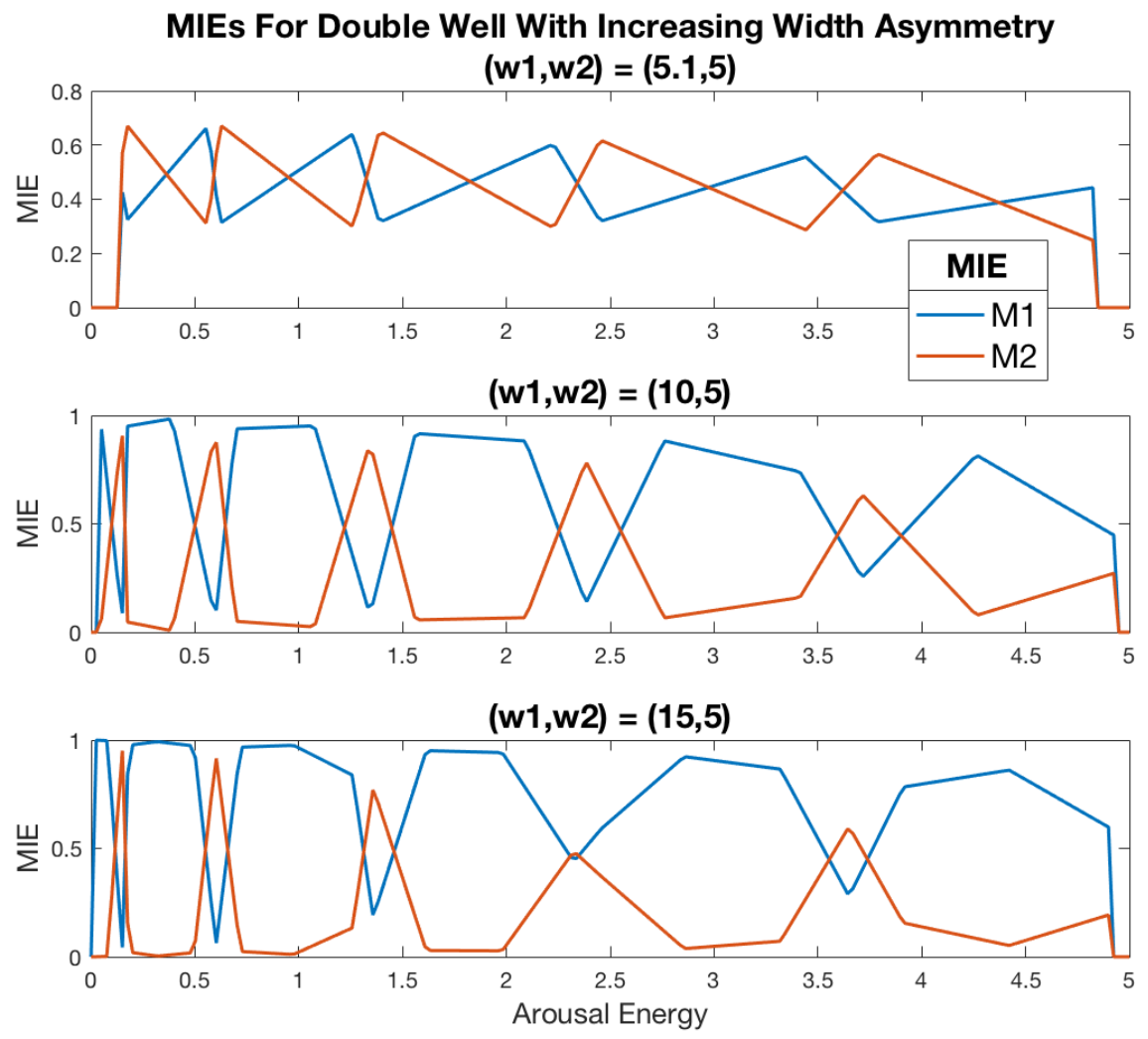
similar, even if both are equally efficacious and fluctuations in arousal remain the same. This is one example of how, under the MPMW, "noise" and conflict

are inextricably tied to both stimulus properties and the agent's level of arousal. In the remainder of this section, we discuss how efficacy asymmetry can be used to overcome asymmetries in representational generality

\subsection{Increasing Efficacy to Overcome Asymmetries in Generality}

Up to this point, we have only addressed landscapes that are asymmetric in either depth or width. However, it is intuitive to expect that, in a system where the target stimulus is less general than the non-target stimulus, the resulting processing bias may be rectified by either an increase in salience of or allocated attention to the target stimulus. It is also intuitive to expect that, the more general the non-target stimulus is, the less impactful differentially allocated efficacy will be. In short, it becomes harder to effectively process information from a target stimulus as the competing stimulus becomes more semantically general.

To formally test this intuition, we calculated drift rate in the lower region of the working range for a set of initially symmetric landscapes at different depth and width asymmetries. We chose five "reference widths", static widths for the target well, for each of which the non-target well would have ten widths, increasing from a width equal to the target well to twice the width of the target well. For each of these fifty sets of widths, we calculated the mean drift rate in the lower range of energies at twenty-five depth asymmetries, wherein the depth of the target well increased from equal to the depth of the non-target well to twice its depth.

Just as in the case of width-symmetric depth-asymmetric landscapes, we found that, on average, drift increased as a function of depth asymmetry for all fifty landscapes, effectively, mean $\left(\frac{d M}{d\left(d_{1} / d_{2}\right)}\right)>0$. Therefore, for all landscapes, increasing the depth of the target well produced a band of energies in which the target well dominated integration and increasing the depth of the target well increased the mean drift rate in that band. Additionally, the increase of drift rate as a function of depth asymmetry was, on average decreasing, as a function of width asymmetry for $84.44 \%$ of the landscapes. That is, for $84 \%$ of landscapes, as the non-target well became more general, the same depth increase for the target well yielded a lower drift rate in the 
Figure 21: Energy bands for width and depth asymmetric double well landscapes. Note that increasing depth asymmetries produces a similar effect in a width asymmetric landscape as it does in a depth asymmetric one. efficacy may, therefore, be used to overcome generality asymmetries, but is only effective in doing so if the agent can remain within the correct level of arousal.
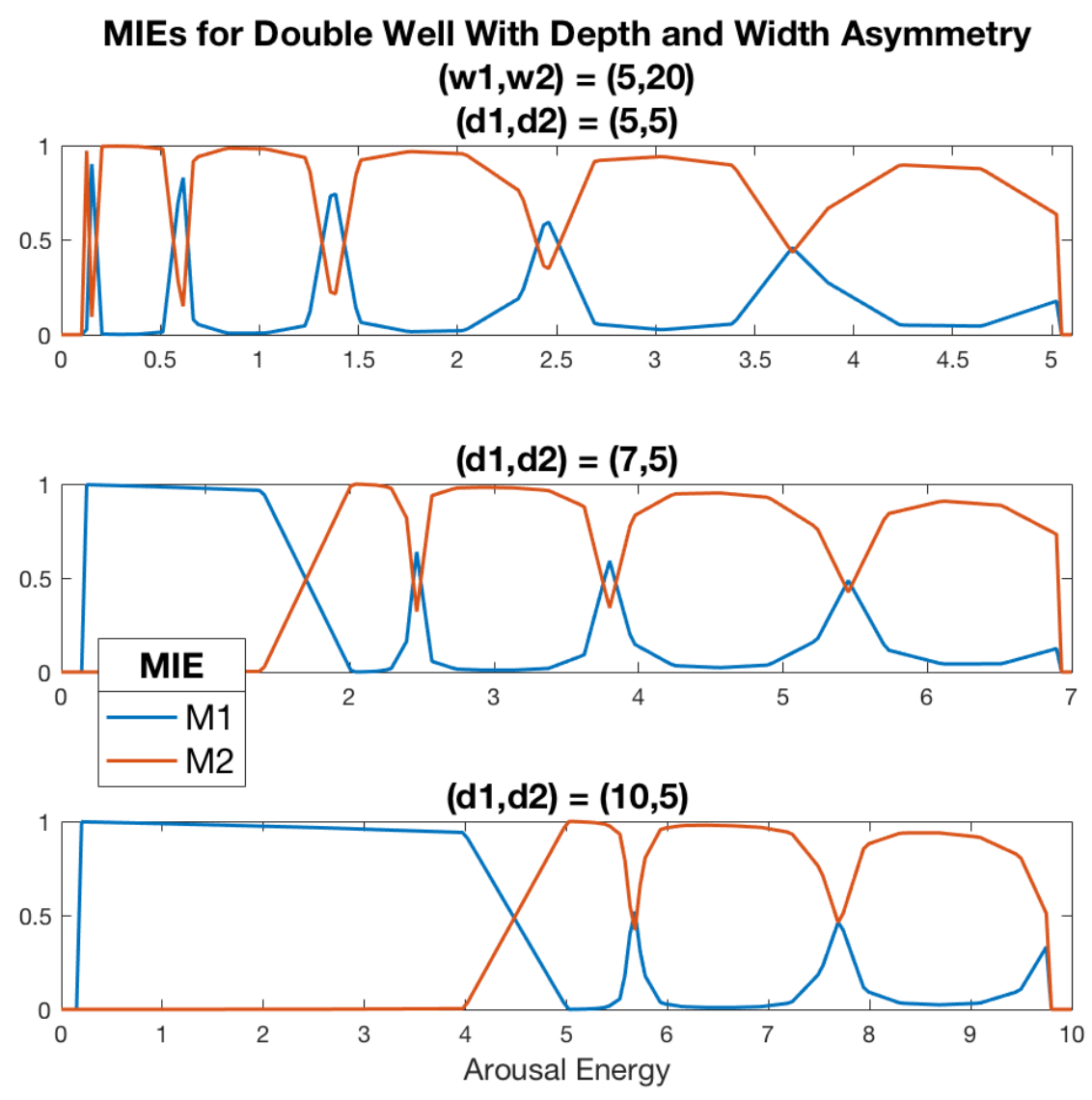
lower band of energies. This means that, in general, it takes more efficacy to bias processing away from a more general non-target stimulus.

This intuitive result helps serve to validate the MPMW as a framework capable of unifying a wide range of cognitive phenomena by reproducing familiar effects addressed by other cognitive models.

\subsection{Conclusion: The Double Well Landscape}

Our analysis of the MPMW in a double well landscape produces a wide range of results that may be applied to $2 \mathrm{AFC}$ task modeling or generalized to a larger number of competing stimuli.

Within the MPMW, the double well landscape offers a richer, more realistic model of how stimulus generality and efficacy allocation effect the information integration process when multiple task-relevant stimuli are present. By assigning task-irrelevant stimuli to the classically forbidden region and task-relevant non-target stimuli to the non-target well ("well 2"), the double well landscape offers two sources of error in the information integration process. In addition to dropping a bit due, which slows the decision process but does not change the direction of the decision-variable's trajectory, the agent may integrate information in favor of the non-target stimulus, which reverses the direction of the drift rate (for a complete treatment of decision-variable dynamics, see [48]). Enough of these types of integration error can produce either a non-response error or an erroneous (incorrect) response, respectively.

Stimuli with equal efficacy and generality exhibit maximum conflict in the form of equal MIEs occurring in closely spaced pairs of energies, producing an average drift rate $\left(M_{1}-M_{2}\right)$ of zero. In general, eigenstate distributions respond to depth and width asymmetries similarly in a double well landscape as they do in a single well landscape. Performance may be biased in favor of a stimulus either by increasing its relative efficacy, its relative generality, or both.

Large depth asymmetries bifurcate the working range of energies into a lower and higher subrange of energies. The lower range will be associated with a larger bandwidth and higher MIE for the deeper well as depth asymmetry increases. The higher range of energies will be associated with larger bandwidths and larger MIEs associated with the shallower well. This predicts a surprising "all or nothing" effect on integration in favor of the deeper well that grows more pronounced as depth asymmetry increases. This means that an agent wishing to leverage controlled attention to overcome general- 
ity asymmetry must allocate sufficient attention to place the bulk of their arousal range within the lower band of energies in order to benefit from the efficacy asymmetry, and might experience the reverse of their intended effect if they fail to do so. Furthermore, the MPMW states that efficacy is more impactful when applied to a more fluent stimulus and less impactful when applied to overcome a more fluent stimulus.

Increasingly large width asymmetries produce increasing bandwidths and, within bands, higher MIEs (therefore, higher magnitude of drift rate) favoring the wider well. This effect denotes that larger width asymmetries, where the wider well is associated with the non-target stimulus, will require a larger depth asymmetry to overcome.

Taken together, the MPMW's predictions about information integration in a double well system capture a wide variety of phenomena. Among these phenomena, the MPMW can reproduce stability-flexibility tradeoffs addressed by task-switching models (see, [39], [27], [26] by assuming that finite time is required to allocate a finite amount of efficacy. The MPMW also predicts a second type of stability-flexibility tradeoff related to arousal. This stability-flexibility tradeoff states that efficacy may be allocated to leave an agent optimally sensitive to information from non-target sources as a result of different-sized arousal fluctuations that may arise naturally. Furthermore, the MPMW states that failure or inability to solve this tradeoff in certain contexts will produce behaviors termed "hyperfocus" and "hypofocus", aspects of which are familiar from ADHD research [3],[15], [35].

\section{Landscapes with Greater Than Two Wells}

One of the attractions of the MPMW framework is its ability to address multichoice decisions by simple extension, with minimal loss of analytic tractability. The general forms of the eingenstates for a square well landscape discussed in section 3.2 hold true for any number of wells and, by solving the TISE by the Matrix Numerov Method, as discussed in Appendix B, the MPMW can be solved for any number of competing stimuli. Many of the general properties of eigenstate distributions described for single and double well landscapes hold true for n-well landscapes. However, there are conditions under which they deviate qualitatively, in particular as a function of the spatial arrangement of wells with respect to one another.

As with a double-well landscape, the N-well landscape potential is equal 
everywhere between wells (barrier regions) and outside of the landscape of interest. However, how the parameters of the wells are distributed (i.e., their relative spacing, width and depth) can have interesting effects, that go beyond those of a double-well landscape. We begin by discussing periodic landscapes, defined as landscapes in which all wells have equal width, depth, and separation, then move on to considering landscapes that deviate from periodicity.

\subsection{Periodic Landscapes on a Straight Line}

As in the double-well landscape, periodic landscapes of $n$ wells exhibit eigenstates that appear in very closely spaced sets of $n$, within which the mean MIEs across the set of $n$ eigen energies are equal for all wells. However, unlike a double-well landscape, individual eigenstates are not distributed equally across all wells. Instead, all PDFs are symmetric about the center of the landscape, resulting in each non-central well having a partner well that is equidistant from the center of the landscape with which it has equal MIEs and is, therefore, most closely in competition. For each eigenstate, the probability that the particle is found in either of these two wells is always equal. Interestingly, this suggests that greatest conflict in a linear $n$-well landscape is between stimuli equidistant (in representation and, sometimes, spatially) from a central stimulus, rather than between closest neighbors as one might expect in a classical system. Evidence for PDFs exhibiting such central parity was found in [63], in which several sequential sampling models were tested for their ability to model the Eriksen-Flanker task. The model that best reproduced behavioral data was a single process model with a continuous PDF exhibiting central parity. This central parity implies that the use of linear landscapes is most appropriate when modeling stimuli distributed similarly. Stimuli more commonly represented otherwise (such as colors or multistable stimuli) may be modeled by introducing periodic boundary conditions (addressed in [44], in which case, the landscape of interest resides on a ring.

Within each set of $n$ energies, each well has $\lceil(n / 2)\rceil$ unique MIEs distributed symmetrically about the median energy for the set. Because the sets of eigenstates are so closely spaced relative to the overall depths of the wells, requiring only very tiny fluctuations in arousal to move between them, it is reasonable to assume that arousal falling within such a cluster of eigenstates the system will spend time occupying all of those states. This will result in a small drift rate for a decision variable comparing evidence accumulated 
for pairs of alternatives or a decision variable comparing evidence between one and the average of the remaining accumulators in a perfectly periodic landscape, despite the fact that the eigenstates are not evenly distributed as they are in a symmetric double-well landscape.

In conclusion, linear n-well landscapes exhibit central parity, with high competition among wells equidistant from center, and lower competition among wells that neighbor one another. This makes them best suited to modeling stimuli that are similarly physically distributed.

Deviations in Periodicity: Barrier Width Introducing a large separation between wells of an otherwise periodic landscape has the effect of dividing the landscape into sub-landscapes on either side of the barrier. The left landscape of $p$ wells and right landscape of $q$ wells will then each behave as though they were independent landscapes of size $p$ and $q$ respectively, but with their eigen energies interleaved together so that small perturbations in arousal will change the sub-landscape (subset of wells) that dominates integration. The extent to which the sub-landscapes are independent from each other (i.e., associated eigenstates are localized to each) depends on the width of the barrier and the eigenstate. Wider barriers produce more independent landscapes and low energy eigenstates will be more tightly localized to one sub-landscape whereas higher energy eigenstates will be more distributed and exhibit greater conflict.

As barrier width reflects representational differentiability, multiple barrier widths may be employed in modeling decision processes involving multivalent stimuli, such as the Stroop task. A treatment of this is addressed in Appendix $\mathrm{C}$ below, wherein a 6 well landscape was used to represent stimuli with both a "color" and "word" component, and three alternatives (red, green, or a neutral control stimulus) for the semantic identity of each. The stimulus components with shared semantic identity (e.g., green ink and the word "green") share a sub-landscape, as they are separated from one another by a smaller barrier than stimulus components with which they do not share a semantic identity (e.g., red ink and the word "red"). This produced asymmetry in the facilitative effects of congruent stimuli and the slowing effects of incongruent stimuli. See Appendix C for greater details of the implementation. 
Figure 22: In orange: a generating periodic landscape. In blue: the first four PDFs of that landscape. All curves are re-scaled for visibility. Note that eigenstates exhibit greatest conflict between wells symmetrically distributed about the center point.

First Four PDFs of A Periodic Landscape

(Rescaled For Legibility) PDF $_{1}$
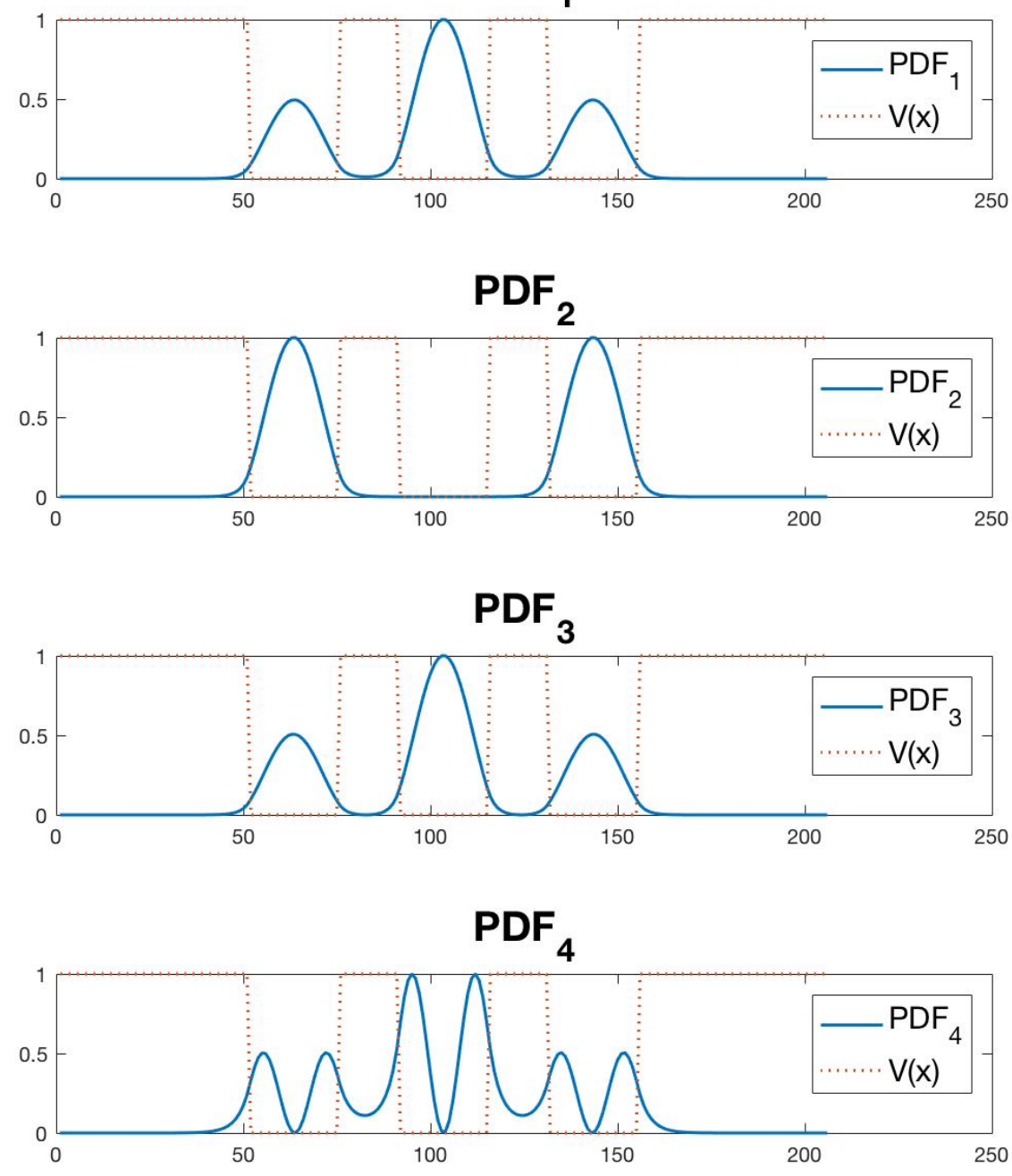
Deviations in Periodicity: Well Depth and Width Deviations from periodicity produce nonlinear localization effects, similar to those exhibited by double well landscapes (see Section 6). However, when one well deviates significantly in depth and/or width from others in an otherwise periodic landscape, it has a similar effect on the surrounding landscape to introducing large barriers on either side: dividing the surrounding landscape into sublandscapes on either side of the non-uniform well. Lower energy eigenstates are localized to each sub-landscape or well, and higher energy eigenstates are more distributed across all wells and exhibit greater conflict. However, even in lower energy eigenstates, the "sub-landscapes" will exhibit some conflict among one another. In particular, sub-landscapes of equal depths will exhibit greater conflict with one another than those of unequal depths. An example, of a landscape and several of its eigenstates is shown in figure 23.

\section{Discussion and Conclusion}

In this article we have addressed the quantum theoretical underpinnings of the MPMW framework introduced in [47] and [48] by examining interactions between well width, depth, and system energy in a variety of landscapes. We have demonstrated how the MPMW framework can be used to model the effects of generality, efficacy, and arousal on performance in information integration tasks by reproducing the standard Stroop effects by replicating the Stroop Model from [17] (see C. Within this framework, we have shown how effects such as hypoarousal, hyperarousal, flow, conflict, stability-flexibility tradeoffs, and stochasticity are emergent properties of the MPMW.

We have shown that the MPMW framework, applied to a single stimulus (or task), provides a formal account of interactions between generality, efficacy and arousal, predicting several major effects. First, efficacy can counteract hyperarousal but, surprisingly, exacerbate the effects of hypoarousal. Additionally, an excess of efficacy can produce disengagement from a task if arousal falls into the hypoarousal range. This effect may motivate agents subject to increasing, uncontrolled efficacy (as by salience increase) to either increase arousal or decrease controlled attention to avoid disengagement. Furthermore, "quantum leaps" in MIE that occur as efficacy increases may motivate exponential and discrete increases in allocated attention or, where controllable, salience. Additionally, increased generality can effectively counteract the effects of hypoarousal but much less so hyperarousal. Increasing 
Figure 23: In orange: a generating landscape wherein a single well deviates from periodicity in depth, producing sub-landscapes one (two wells), two (one well) and three (four wells). In blue: the first four PDFs of that landscape. All curves are re-scaled for visibility.

\section{PDFs of Aperiodic Landscape (Rescaled for Legibility) \\ PDF $_{1}$}
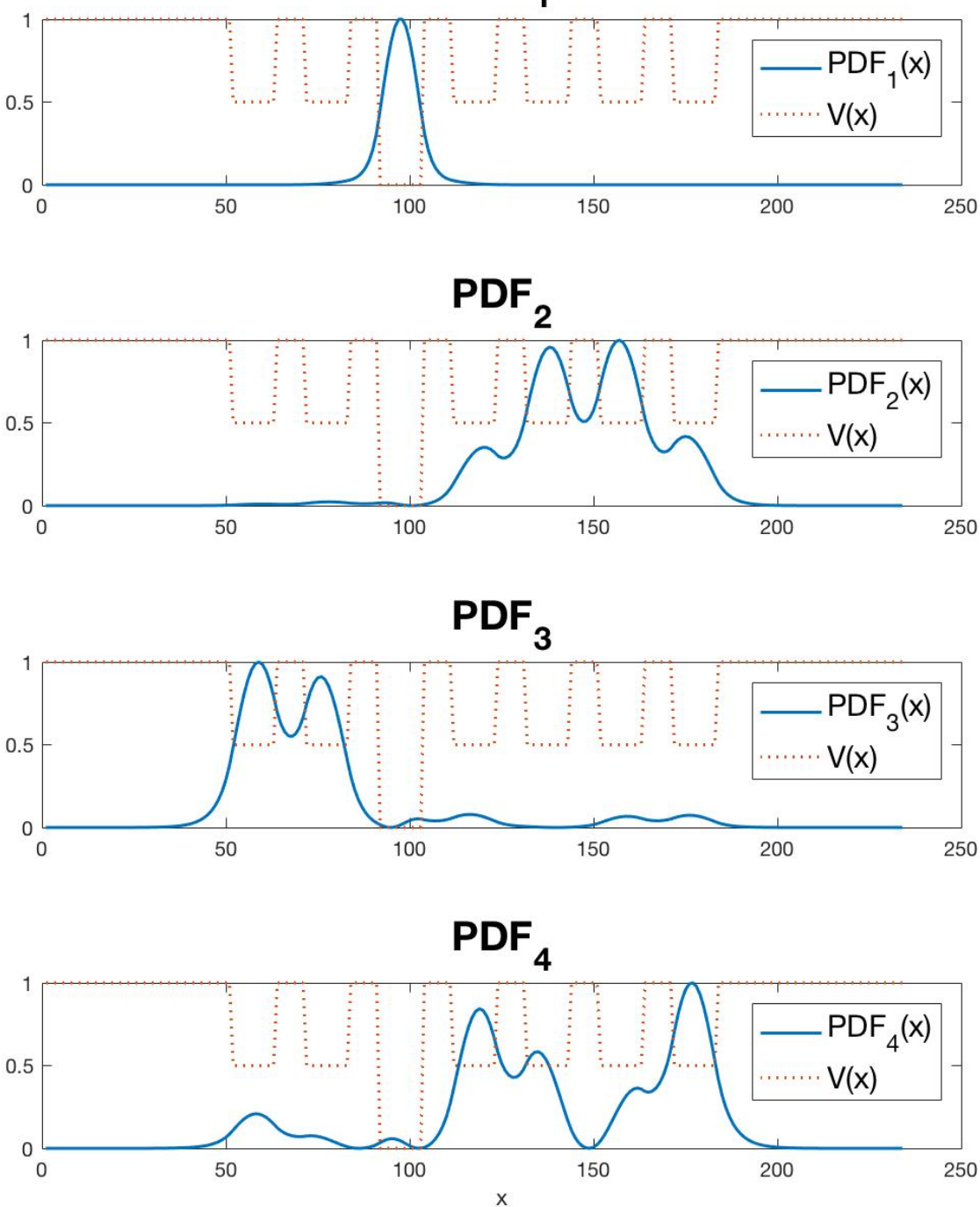
generality also produces "quantum leaps" in performance parameters that may motivate linearly spaced discretization of continuous stimuli.

We have also shown that small deviations from symmetric efficacy or generality produce bands of arousal energies in which information integration is dominated almost entirely by one stimulus, and that these bands become wider and performance more heavily biased as asymmetry increases. One surprising property that follows from this is that, though agents may differentially apply controlled attention to increase efficacy of a target stimulus and bias processing in its favor, arousal must remain in the low end of the working range of energies for the desired effect to be attained. Arousal that exceeds the low end of the working range can result in the reversal of the desired effect, biasing processing in favor of the non-target stimulus. This property gives rise to an arousal-centric stability-flexibility tradeoff, in which the correct level of controlled attention must be allocated to the target stimulus to render processing sufficiently robust to arousal fluctuations, but not so robust that processing cannot be redirected when appropriate (hyperfocus). Additionally, we have shown that the MPMW framework captures a wide variety of commonly disparate effects that are addressed by classical models. Among these effects, we have shown that for any number of stimuli (or tasks), the impact of controlled attention as a mechanism by which conflict is reduced and information integration flexibly biased is limited by the relative generality of stimuli or tasks. We have also addressed the more familiar stability-flexibility tradeoff, wherein the reallocation of controlled attention (impacting efficacy) from one stimulus to another will incur a switch cost .

Finally, we have shown that the MPMW can be applied to any number of stimulus-response mappings by simply introducing as many wells within the landscape as exist task-relevant stimulus-response pairings. This allows the MPMW to expand naturally to handle N-alternative forced choice, which requires solving a more complex TISE, but that can rely upon the same methods (see B) as for a single or double well landscape.

There are several directions to be addressed in future work. One branch focuses on comparing decision-making performance across different types of "race" for N-alternative forced choice. One focuses on expanding the MPMW to include multiple levels of processing for the purpose of modeling taskswitching processes for tasks with competing stimulus components. Finally, a third addresses flexible reallocation of efficacy on both within- and betweentrial timescales. 


\section{A A Formal Analysis of the Evidence Accu- mulation Process for a Single Well System}

In this section, we provide a formal derivation of probabilities of response and response times for single well landscapes, (i.e., signal detection tasks), using similar methods to those employed in [48], and a brief illustration of how energy effects decision times.

An agent who is working in a single alternative forced choice perceptual decision making task is simply choosing when to "act", which will transition them out of their default state, "do not act". For the example pedestrian, the decision is when to "cross the street". Failure may occur in one of two ways: by failing to respond to the stimulus sufficiently quickly (e.g., not recognizing the "walk" signal until it's too late to complete the crossing before it changes) or responding as if the stimulus were present when it is not (e.g., being presented with a "do not walk" signal and attempting to cross as though it were a "walk" signal). Treatment of the second case requires the presence of more than one well, and therefore is outside of the scope of this section.

We model this process using a Markov decision variable $x(t)$ that begins in state $x(0)=0$. The state of $x(t)$ is given by the sum over the bits of information have been integrated as evidence in favor of action. The decision is made once $x(t) \geq T_{s}$. At each point in time, incident information arrives as a discrete packet of information, simultaneously containing stochastically distributed evidence for both alternatives ("act" and "do not act"), the distribution of which is given by the PDF associated with the landscape and state of arousal (a linear combination of the bound states for the system). Each bit of evidence is categorized by a measurement process, thus collapsing its state to occupy either the well or the classically forbidden region. This models each time step as a bernoulli random variable and, therefore, the state of the system by a binomial distribution.

Supposing arousal, $E$, is constant the probability that each bit is categorized as evidence for "act" is given by its associated $M_{1}$ and the probability of categorizing it as evidence for "do not act" is given by $1-M_{1}$.

The single step transition matrix for the decision variable $x(t)$ is given by 
a square matrix of the dimensions $\mathbb{R}^{T_{s} \times T_{s}}$

$$
T_{\text {single }}=\left[\begin{array}{ccccc}
1-M_{1} & M_{1} & 0 & \ldots & 0 \\
0 & 1-M_{1} & M_{1} & \ldots & 0 \\
0 & 0 & 1-M_{1} & \ldots & 0
\end{array}\right]
$$

where $T_{s}$ is the threshold to act. The transition matrix governing the entire decision process is generated by embedding $T_{\text {single }}$ in the larger matrix $T \in \mathbb{R}^{\left(T_{s}+1, T_{s}+1\right)}$, wherein an absorbing state is assigned to the state at $T_{s}$.

$$
T=\left[\begin{array}{cc}
T_{\text {single }} & m \\
0 & 1
\end{array}\right]
$$

where $m$ is a column vector in $\mathbb{R}^{T_{s}}$ with all but the last entry 0 and last entry $M_{1}$.

The response time distribution is given by the product

$$
R T(t)=s_{0}\left(T^{t}-T^{t-1}\right) s_{a}=M_{1}\left(T_{\text {single }}^{t-1}\right)_{\left(1, T_{s}\right)}
$$

where $s_{0}$ is the basis vector in $\mathbb{R}^{1 \times T_{s}+1}$ representing the state $x(0)=0$ and $s_{a}$ is the basis vector in $\mathbb{R}^{T_{s}+1 \times 1}$ representing the state $x(t)=T_{s}$. Finally, $\left(T_{\text {single }}^{t-1}\right)_{\left(1, T_{s}\right)}$ is the first row and final column of $T_{\text {single }}^{t-1}$. This value may be found by following the same procedure as in [48].

Because $T_{\text {single }}$ is a Jordan matrix, $T_{\text {single }}=M_{1} J\left(\frac{1-M_{1}}{M_{1}}\right)$, we can find the entries of its powers explicitly. By properties of Jordan matrices, the RT distributions are given by

$$
R T(t)=\left[\left(\frac{(t-1) !}{\left(t-T_{s}\right) !\left(T_{s}-1\right) !}\right)\left(1-M_{1}\right)^{t-T_{s}}\left(M_{1}\right)^{T_{s}}\right]
$$

and expected response time is given by

$$
\mathbb{E}(R T)=\sum_{i=t}^{\infty} R T(t)=\frac{T_{s}}{M_{1}}
$$

and probability of acting within a given time limit is given by

$$
P(\text { act })=\sum_{t=1}^{\text {time limit }} R T(t)
$$


As arousal increases, so does the probability of dropping a bit. As a result, higher energies correspond to higher probabilities of non-response and longer response times when the agent does respond. This is illustrated in figure 24 .

Figure 24: Probability of response and mean response times for a signal detection (single well) task. Well depth is 1 and width is 50 . Threshold is 5 and time limit is 7 .
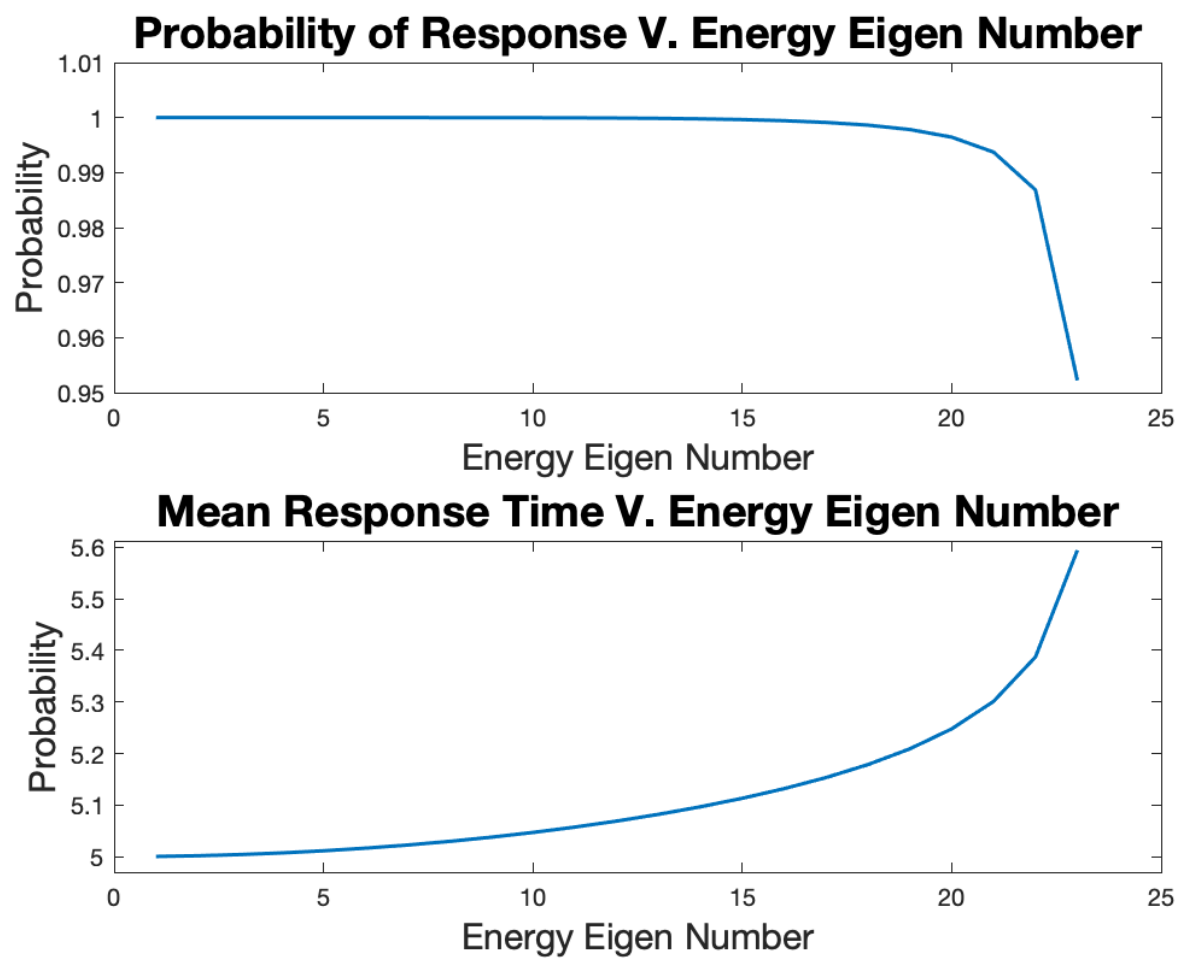

In [48] we established that the state of a system of $m$ integrators, applied in parallel to the same task and integrating information from identical sources, at time $t$ will be identical to the state of a single integrator system at time $m t$. Extended analysis of RT distributions for a system of $m$ integrators is not needed here as it follows directly from the analysis performed above. We can, however, state that the expected RT for such a system is given by

$$
\mathbb{E}(R T)=\frac{T_{s}}{m M_{1}}
$$

but is still bounded by a minimum of $t=1$ as that is the minimum 
amount of time required for any number of integrators to process a bit of information.

\section{B Numerical Methods for Solving the TISE}

In order to find the MIE for an alternative $\left(M_{k}\right)$, we are required to solve the TISE. The MIEs are given by equation 14 and

$$
\begin{gathered}
M_{k}=\sum_{j}^{J} \frac{1}{a_{j}} \int_{l_{k}}^{u_{k}}|\psi(x)|^{2} d x \\
=\sum_{j}^{J} \frac{1}{a_{j}} M_{k, j}
\end{gathered}
$$

where $j$ denotes the index of a bound state, $a_{j}$ is a normalizing constant determined by some PDF describing the probability that a particle occupies a certain bound state, $l_{k}$ is the lower bound of well $k$ and $u_{k}$ is the upper boound of well $k . M_{k, j}$ describes the probability that a particle is found in well $k$ given bound state $j$. Note that, because $\psi_{j}(x)$ is non-zero in regions outside of the wells,

$$
\sum_{k=1}^{\eta} M_{k}<1
$$

In our treatment of the $2 \mathrm{AFC}$ problem ([47]) we assumed that all bound states were equally probable, so

$$
M_{k}=\sum_{j=1}^{J} \frac{1}{J} \int_{l_{k}}^{u_{k}}\left|\psi_{j}(x)\right|^{2}
$$

where $\eta$ is the total number of wells. Here, however, we relax that assumption, and do not place a requirement on the range of bound states a particle may occupy, we require only that it does occupy some bound states.

There are very few circumstances under which the TISE may be analytically solved and many methods developed to solve it numerically solve only for a small number of the lowest energy bound states. In fact, solving the TISE for all bound states of a system is a non-trivial problem and not all methods of solving the TISE are designed to do so for all bound states. 
In this section, we present two methods of performing this task, the Matrix Numerov Method [44] and the Matrix Marsiglio Method [34]. Both methods require embedding the landscape of interest within a well of infinite depth but finite width, outside of which $\psi(x)=0$. This is an approximation of the boundary conditions given by equation 2 that allows the theoretically infinite system to be truncated to a finite one, thus allowing the TISE to be solved by the use of finite matrices. In order to yield accurate solutions, the width of the embedding landscape must be determined carefully, as it forces the state functions to collapse to 0 at a finite distance from the landscape of interest, introducing errors in the calculated eigen energies and their associated eigenstates. Widening the portions of the embedding well to either side of the landscape reduces these errors, but makes solving the TISE more computationally intensive, as the matrices involved grow larger.

\section{B.1 Matrix Numerov Method}

The Matrix Numerov Method ([44]) is a simple matrix method, under which the potential, $V(x)$ is discretized with a step size determined by the minimum wavelength of the system's bound states,

$$
\lambda=\frac{h}{\sqrt{2 m V_{\max }}}
$$

so that step size is given by

$$
d=\frac{\lambda}{2 \pi}
$$

This step size is recommended by the authors of [44], but we often use a step size

$$
d=\frac{\lambda}{8 \pi}
$$

for graphing and modeling purposes, as it significantly improves the "resolution" of the potential and therefore the accuracy of the computed eigen energies and eigenstates.

The authors also recommend that approximately $2 \lambda$ of space is left on either side of the landscape of interest to allow for proper convergence of the eigenstates. The length of the vector for the discretized landscape $V$ is then given by rounding

$$
N=\frac{x_{t}}{d}+4 \pi
$$


where $x_{t} / d$ describes the number of steps required capture the width of the landscape of interest This determines the size of the matrices used to write the TISE as

$$
-\frac{\hbar^{2}}{2 m}\left[B^{-1} A+V\right] \psi=E \psi
$$

$A$ and $B$ are square symmetric matrices given by

$$
A=\frac{1}{d^{2}}\left[\begin{array}{ccccc}
-2 & 1 & 0 & \ldots & 0 \\
1 & -2 & 1 & \ldots & 0 \\
0 & 1 & -2 & \ldots & 0
\end{array}\right]
$$

and

$$
B=\frac{1}{12}\left[\begin{array}{ccccc}
10 & 1 & 0 & \ldots & 0 \\
1 & 10 & 1 & \ldots & 0 \\
0 & 1 & 10 & \ldots & 0
\end{array}\right]
$$

and $V$ is simply a diagonal where the diagonal elements are given by the discretized potential vector. This simple equation gives all bound states of the system and their energies as eigenvectors and eigenvalues of equation 17.

The Matrix Numerov Method is intuitive and computationally cheap under the correct circumstances. However, its method of system discretization means that the size of its errors are determined by step size, and the state functions it creates are not truly smooth or continuous, as required by the boundary conditions of the TISE. To rectify these issues, one may employ the Matrix Marsiglio Method.

\section{B.2 The Matrix Marsiglio Method}

The general form of the Matrix Marsiglio method can be found in [34]; in this section, we only address its application for the MPMW. As in the Matrix Numerov Method, the Matrix Marsiglio Method also uses an infinite embedding well to drive the convergence of the eigenstates to zero, but it differs in that it operates on continuous equations by framing the eigenstates of the landscape of interest as linear combinations of the eigenstates of the embedding well. Whereas, in the Matrix Numerov method, errors arise from the number of steps into which the landscape is discretized, errors in the Marsiglio method arise from the number of eigenstates of the infinitely deep embedding well contribute to the formulation of the eigenstates of the landscape of interest. These methods converge on similar sources of error in that 
both can yield nearly exact solutions when given sufficiently many terms, and the performance of each is bounded by the number of terms required to yield a solution within a certain level of accuracy. In both cases, the number of terms defines the size of a matrix whose eigenvalues and eigenvectors must be found, a process that forms the bulk of the computational demands for these methods.

To formulate the solutions to the TISE using the Marsiglo Method, we write each of the eigenstates of the landscape of interest as,

$$
\psi(x)=\sum_{n=1}^{\infty} c_{n} \phi_{n}(x)
$$

where $\phi_{n}(x)$ is the $n^{t h}$ of infinitely many eigenstates of the embedding well,

$$
\phi_{n}(x)=\sqrt{\frac{2}{a}} \sin \left(\frac{n \pi x}{a}\right)
$$

and $a$ is the width of the embedding well. For the purpose of this article, we chose $a$ to be the sum of the widths of all wells and separating barriers that comprise the landscape of interest, plus the two surrounding barriers with widths determined by the same method as in the Matrix Numerov Method. An exact solution would require infinitely many terms to contribute to this sum. However, truncation allows for accuracy within an arbitrary margin of error.

Therefore, where $\psi_{j}$ is the $j^{\text {th }}$ eigenstate of the landscape of interest and $\phi_{n}$ is the $n^{t h}$ eigenstate of the embedding landscape.

$$
\psi_{(j)}(x)=\sum_{n=1} c_{n}^{(j)} \sqrt{\frac{2}{a}} \sin \left(\frac{n \pi x}{a}\right)
$$

The terms $c_{n}^{(j)}$ are the $n^{\text {th }}$ terms of the $j^{\text {th }}$ eigenvector of a matrix $H$, such that

$$
H c^{(j)}=E_{j} c^{(j)}
$$

and $E_{j}$ is its $j^{\text {th }}$ eigenvalue. The matrix $H$ has terms $H_{p, q}$ described as follows 


$$
H_{p, q}=\delta_{p, q} \frac{1}{2} p^{\prime 2}+\frac{2}{a} \int_{0}^{a} V(x) \sin \left(p^{\prime} x\right) \sin \left(q^{\prime} x\right) d x
$$

where $p^{\prime}=p \pi / a$.

The solutions of this equation are made far less complex in our use case by the fact that the landscape is piecewise constant, allowing the integral to be converted to a sum over a set of integrals, each scaled by the value of $V$ in each area of the landscape. We will describe them for a landscape with $N$ attractor wells of any depth or width.

In such a landscape, there are $2 N+1$ regions, including all interstitial barrier regions and the barriers that sit between the landscape of interest and the walls of the infinite embedding well. We denote each region by index $r$ and, beginning indexing from 1, barriers occupy odd-indexed regions and have potential $V$ (barrier $)=V_{\max }$. Attractor wells occupy odd-indexed regions and each well $k$ has its own depth, $V\left(\right.$ well $\left._{k}\right)=V_{\max }-d_{k}$. We define the bounds by a set $b$ (of length $2 N$ ) so the upper bound of well $k$ is denoted by $x=b_{2 k}$ and the lower bound is denoted $x=b_{2 k-1}$. The potential landscape is therefore defined piecewise as,

$$
V_{r}= \begin{cases}\infty & \text { if }[x<0, x>a] \\ V_{\max } & \text { ifrisodd } \\ V_{\max }-d_{k} & \text { ifriseven }\end{cases}
$$

where $r$ is the index of the region.

The terms $H_{p, q}$ can be found by using a few algebraic tricks. First, we recognize that the integral

$$
\begin{gathered}
\frac{2}{a} \int_{0}^{a} V(x) \sin \left(p^{\prime} x\right) \sin \left(q^{\prime} x\right) d x= \\
\frac{2}{a}\left(\int_{0}^{a} V_{\text {max }} \sin \left(p^{\prime} x\right) \sin \left(q^{\prime} x\right) d x-\sum_{k=1}^{N} \int_{b_{2 k-1}}^{2 k} d_{k} \sin \left(p^{\prime} x\right) \sin \left(q^{\prime} x\right) d x\right)
\end{gathered}
$$

then, for the on-diagonals, where $p=q$, we have

$$
\begin{gathered}
\frac{2}{a} \int_{0}^{a} V(x) \sin \left(p^{\prime} x\right) \sin \left(q^{\prime} x\right) d x= \\
\frac{2}{a}\left(\int_{0}^{a} V_{\max } \sin ^{2}\left(p^{\prime} x\right) d x-\sum_{k=1}^{N} \int_{b_{2 k-1}}^{2 k} d_{k} \sin ^{2}\left(p^{\prime} x\right) d x\right)
\end{gathered}
$$


where we can leverage the trigonometric identity

$$
\sin ^{2}(x)=\frac{1}{2}(1-\cos (2 x))
$$

to find that

$$
\int \sin ^{2}(x) d x=\frac{1}{2}(x-\sin (x) \cos (x))
$$

and, through a bit of arithmetic, we can reduce the on-diagonal terms of $H$ from an integral, which are computationally demanding to compute, to a simple sum given by

$$
\begin{aligned}
H_{p, p}= & \frac{1}{2}\left(\frac{p \pi}{a}\right)^{2}+V_{\text {max }}-\sum_{r=1}^{N}\left(\frac{d_{r}}{2 p \pi}\right)\left[\left(\frac{2 p \pi}{a}\right)\left(b_{2 r}-b_{2 r-1}\right)+\right. \\
& \left.\sin \left((2 p \pi)\left(\frac{b_{2 r-1}}{a}\right)\right)-\sin \left((2 p \pi)\left(\frac{b_{2 r}}{a}\right)\right)\right]
\end{aligned}
$$

For the off-diagonals, the integral is still given by a sum of integrals and we can leverage a different trigonometric identity,

$$
\sin (A) \sin (B)=\frac{1}{2}(\cos (A-B)-\cos (A+B))
$$

to reduce the integral to a sum, given by

$$
\begin{gathered}
\int \sin \left(p^{\prime} x\right) \sin \left(q^{\prime} x\right) d x= \\
-\frac{1}{p^{\prime 2}-q^{\prime 2}}\left(q^{\prime} \sin \left(p^{\prime} x\right) \cos \left(q^{\prime} x\right)-p^{\prime} \cos \left(p^{\prime} x\right) \sin \left(q^{\prime} x\right)\right)
\end{gathered}
$$

and reduce the off-diagonal elements of $H$ to a sum given by

$$
\begin{gathered}
H_{p, q}=\sum_{r=1}^{N} \frac{d_{r}}{\pi}\left[\left(\frac{1}{p-q}\right)\right. \\
{\left[\sin \left(b_{2 r}(q-p)\left(\frac{\pi}{a}\right)\right)-\sin \left(b_{2 r-1}(q-p)\left(\frac{\pi}{a}\right)\right)\right]+} \\
\left.\left(\frac{1}{p+q}\right)\left[\sin \left(b_{2 r}(p+q)\left(\frac{\pi}{a}\right)\right)-\sin \left(b_{2 r-1}(p+q)\left(\frac{\pi}{a}\right)\right)\right]\right]
\end{gathered}
$$


By properties of sines, $H$ is a symmetric matrix. These values allow us to solve the eigenvalue problem given above to produce the eigen energies and eigenstates of the landscape of interest using a sparse representation.

\section{Simulating the Stroop Task}

In order to validate the MPMW on a common psychological task, the Stroop task, we sought to emulate the results found in [17], which were in turn replicating results found in [33] and [16]. In [16], the Stroop stimuli consisted of the names of colors printed in colored ink. The semantic identities were either "RED" or "GREEN", and each stimulus type could also be replaced by a control stimulus that, in simulation, is represented by a "null" input. Agents were asked either to read the word or name the color of the ink in which it was printed. Thus, there were eight possible stimulus combinations: "RED" with either red ink, green ink, or "null" ink, "GREEN" in either red ink, green ink, or "null" ink, or a "NULL" word input with either red or green ink. There were no stimuli in which both the word and color stimuli were null.

To test the MPMW's capacity to recover these results, we constructed the simplest possible model of the decision-making process.

We began by constructing a circular four-well landscape, where the wells represented the response "RED" to an ink stimulus, the response "RED" to a word stimulus, the response "GREEN" to an ink stimulus, and the response "GREEN" to a word stimulus. As each semantic identity ("RED" or "GREEN") is equally general, all four wells were of equal width. Within the larger landscape, we constructed two sub-landscapes, corresponding to the two unique semantic identities "RED" and "GREEN" by placing the two wells corresponding to each semantic identity close to one another, and separating the pairs of wells by a larger barrier. Each well can be identified by an ordered pair indicating the stimulus type (WORD or INK) and semantic identity (RED or GREEN). From radial positions 0 to $2 \pi$ the order of the wells proceeded as follows: WORD, RED, WORD, GREEN, $I N K, G R E E N, I N K, R E D$, so that each stimulus was neighbored on one side by a stimulus sharing one of its components (words next to words, greens next to greens, and so on).

As a reflection of the relative automaticity of tasks, wells associated with word reading were deeper than those associated with color naming. Upon 
each trial, well depth was increased by a constant multiple for wells associated with the task at hand and wells associated with the stimulus presented on that trial. For example, on a "word reading task", the cue ("word reading") that an agent would receive prior to stimulus onset was reflected by a proportional increase in the wells associated with word reading. A stimulus (e.g., the word "RED" printed in green ink) would be reflected by a second proportional increase in the depths of the (WORD, RED) well and the (INK, GREEN) well. We assumed that these two increases occurred immediately upon cue and stimulus onset, that no further changes in well depth occurred throughout the course of a trial, and that the entirety of information integration occurred after the wells reached their final depths. In a trial containing a control stimulus, only the well associated with a possible output would increase in depth as a result of the stimulus presentation.

Finally, following convention set in [48], we assumed all bound states to be equally probable, and therefore took $M_{1}$ to be the sum of the average MIEs across all energies for wells associated with the target stimulus (e.g., (WORD, RED) and (INK, RED), in the above example) and $M_{2}$ to be the sum of the average MIEs across all energies for the wells associated with the non-target stimulus (e.g., (WORD, GREEN) and (INK, GREEN), in the above example).

To fit the landscape parameters, we performed a simple parameter search beginning with a set of estimated parameters and parameter relationships (relative depths and separations). For each parameter set, we simulated 50,000 trials. Each trial was randomly chosen to be either a control trial (in which case only the word or color component of the stimulus would be present) or not. Stimulus components were also randomly assigned based on the trial's identity as a control or non-control trial. Each trial proceeded in keeping with the $2 \mathrm{AFC}$ decision process described by [48]. We recorded response times (RTs) for each and found mean RTs for each of six categories, varying along identities as control, congruent, or incongruent trials and task identity. For the fittedn landscape, RT results are as shown in figure 25.

Note that all major effects captured in [17] are present in these results. First, the more automatic task, word reading, exhibited significantly faster RTs than the less automatic task. Second, each task exhibited a facilitative effect when stimuli were congruent (e.g., the word "RED" in red ink) and a conflict effect when stimuli were incongruent. For both tasks the conflict effect effect was larger than the facilitation effect. Furthermore, the less automatic task, color naming, exhibited significantly stronger effects, both 


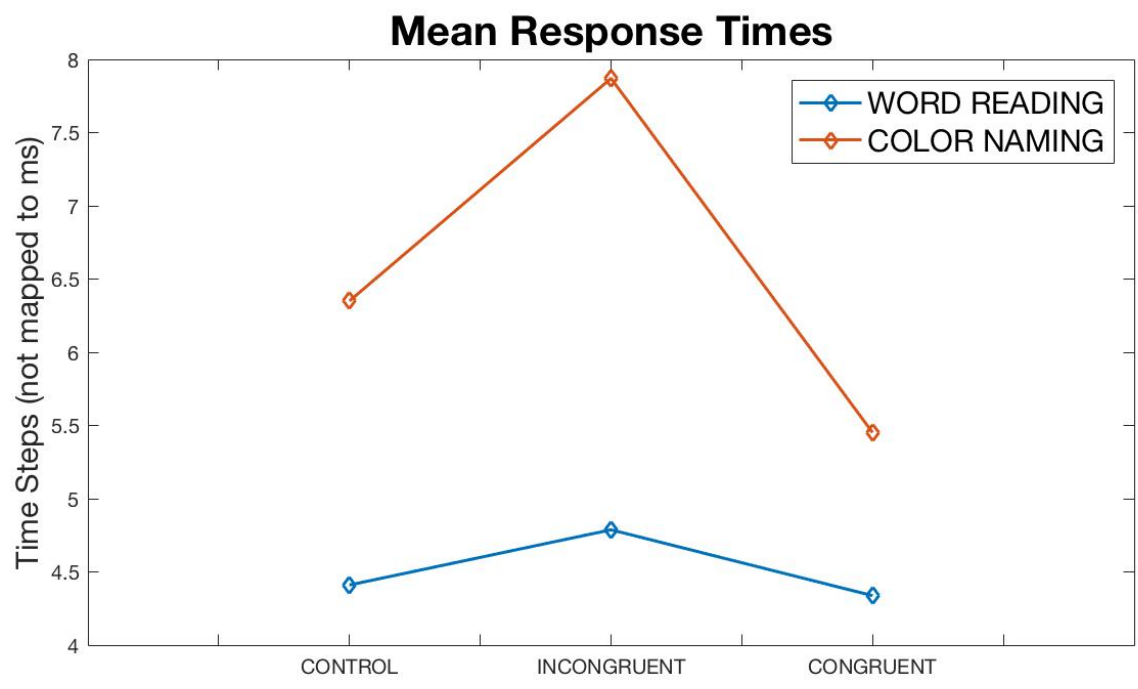

Figure 25: Mean response times in time steps for each of 6 trial types for the simulated Stroop Task.

facilitative and conflict, than the more automatic task.

Upon multiple runs of 50,000 trials, all effects remained the same. These simple simulations serve to validate the MPMW as a framework capable of modeling benchmark results captured by more complex neural network models.

\section{References}

[1] Shakuntala Balaraman. "Color vision research and trichromatic theory: A historical review." In: Psychological bulletin 59.5 (1962), p. 434.

[2] Fuat Balci et al. "Acquisition of decision making criteria: reward rate ultimately beats accuracy". English. In: Attention, Perception, \& Psychophysics 73.2 (2011), pp. 640-657.

[3] Russell A. Barkley. "Behavioral Inhibition, Sustained Attention, and Executive Functions: Constructing a Unifying Theory of ADHD". English. In: Psychological bulletin 121.1 (1997), pp. 65-94. 
[4] Rafal Bogacz. "Optimal decision-making theories: linking neurobiology with behaviour". English. In: Trends in cognitive sciences 11.3 (2006;2007;), pp. 118-125.

[5] Rafal Bogacz and Jonathan D. Cohen. "Parameterization of connectionist models". English. In: Behavior research methods 36.4 (2004), p. 732 .

[6] Rafal Bogacz et al. "The Physics of Optimal Decision Making: A Formal Analysis of Models of Performance in Two-Alternative ForcedChoice Tasks". English. In: Psychological review 113.4 (2006), pp. 700765 .

[7] Matthew M Botvinick et al. "Conflict monitoring and cognitive control." In: Psychological review 108.3 (2001), p. 624.

[8] Timothy J. Buschman and Earl K. Miller. "Shifting the spotlight of attention: evidence for discrete computations in cognition". English. In: Frontiers in human neuroscience 4 (2010), pp. 194-194.

[9] Jerome R Busemeyer, Zheng Wang, and Ariane Lambert-Mogiliansky. "Empirical comparison of Markov and quantum models of decision making". In: Journal of Mathematical Psychology 53.5 (2009), pp. 423433.

[10] Jerome R. Busemeyer and James T. Townsend. "Decision Field Theory: A Dynamic-Cognitive Approach to Decision Making in an Uncertain Environment". English. In: Psychological review 100.3 (1993), pp. 432459.

[11] Jerome R. Busemeyer and Zheng Wang. "What Is Quantum Cognition, and How Is It Applied to Psychology?" English. In: Current directions in psychological science : a journal of the American Psychological Society 24.3 (2015), pp. 163-169.

[12] Jerome R. Busemeyer, Zheng Wang, and James T. Townsend. "Quantum dynamics of human decision-making". English. In: Journal of mathematical psychology 50.3 (2006), pp. 220-241.

[13] Laura Bustamante et al. "Learning to overexert cognitive control in a Stroop task". In: Cognitive, Affective, \& Behavioral Neuroscience 21.3 (2021), pp. 453-471. 
[14] Cameron S. Carter et al. "Parsing Executive Processes: Strategic vs. Evaluative Functions of the Anterior Cingulate Cortex". English. In: Proceedings of the National Academy of Sciences - PNAS 97.4 (2000), pp. 1944-1948.

[15] F. X. Castellanos et al. "Characterizing cognition in ADHD: beyond executive dysfunction". English. In: Trends in cognitive sciences 10.3 (2006), pp. 117-123.

[16] Jonathan D Cohen, Kevin Dunbar, and James L McClelland. "On the control of automatic processes: a parallel distributed processing account of the Stroop effect." In: Psychological review 97.3 (1990), p. 332.

[17] Jonathan D. Cohen, David Servan-Schreiber, and James L. McClelland. "A Parallel Distributed Processing Approach to Automaticity". English. In: The American journal of psychology 105.2 (1992), pp. 239269.

[18] T. Dauphinee and F. Marsiglio. "Asymmetric wave functions from tiny perturbations". English. In: American journal of physics 83.10 (2015), pp. 861-866.

[19] Jochen Ditterich. "A Comparison between Mechanisms of Multi-Alternative Perceptual Decision Making: Ability to Explain Human Behavior, Predictions for Neurophysiology, and Relationship with Decision Theory". English. In: Frontiers in neuroscience 4 (2010), pp. 184-184.

[20] Daniel Durstewitz and Gustavo Deco. "Computational significance of transient dynamics in cortical networks". In: European Journal of Neuroscience 27.1 (2008), pp. 217-227.

[21] Robert H. Edwards. "The Neurotransmitter Cycle and Quantal Size". English. In: Neuron (Cambridge, Mass.) 55.6 (2007), pp. 835-858.

[22] Thomas Goschke. "Intentional reconfiguration and J-TI involuntary persistence in task set switching". In: Control of cognitive processes: Attention and performance XVIII 18 (2000), p. 331.

[23] Gabriele Gratton, Michael G. H. Coles, and Emanuel Donchin. "Optimizing the Use of Information: Strategic Control of Activation of Responses". English. In: Journal of experimental psychology. General 121.4 (1992), pp. 480-506. 
[24] D.J. Griffiths. Introduction to Quantum Mechanics: Pearson New International Edition. Always learning. Pearson Education Limited, 2013. ISBN: 9781292037141. URL: https ://books . google.com/books?id= IySpBwAAQBAJ.

[25] Leo M Hurvich and Dorothea Jameson. "An opponent-process theory of color vision." In: Psychological review 64.6p1 (1957), p. 384.

[26] Andrea Kiesel et al. "Control and interference in task switching-A review." In: Psychological bulletin 136.5 (2010), p. 849.

[27] Iring Koch et al. "The role of inhibition in task switching: A review". In: Psychonomic bulletin \& review 17.1 (2010), pp. 1-14.

[28] Peter D. Kvam, Jerome R. Busemeyer, and Timothy J. Pleskac. "Temporal oscillations in preference strength provide evidence for an open system model of constructed preference". English. In: Scientific reports 11.1 (2021), pp. 8169-8169.

[29] Kenneth W Latimer et al. "Single-trial spike trains in parietal cortex reveal discrete steps during decision-making". In: Science 349.6244 (2015), pp. 184-187.

[30] Gordon D. Logan and N. J. Zbrodoff. "When it helps to be misled: Facilitative effects of increasing the frequency of conflicting stimuli in a Stroop-like task". English. In: Memory $\&$ cognition 7.3 (1979), pp. 166174 .

[31] Artur Luczak, Peter Bartho, and Kenneth D Harris. "Gating of sensory input by spontaneous cortical activity". In: Journal of Neuroscience 33.4 (2013), pp. 1684-1695.

[32] Andrzej Lukasik. "Quantum models of cognition and decision". English. In: International journal of parallel, emergent and distributed systems 33.3 (2018), pp. 336-345.

[33] Colin M MacLeod and Kevin Dunbar. "Training and Stroop-like interference: evidence for a continuum of automaticity." In: Journal of Experimental Psychology: Learning, Memory, and Cognition 14.1 (1988), p. 126.

[34] F. Marsiglio. "The harmonic oscillator in quantum mechanics: A third way". English. In: American journal of physics 77.3 (2009;2008;), pp. 253258. 
[35] Diana Martella et al. "Arousal and Executive Alterations in Attention Deficit Hyperactivity Disorder (ADHD)". English. In: Frontiers in psychology 11 (2020), pp. 1991-1991.

[36] Lucia Melloni et al. "(Micro) Saccades, corollary activity and cortical oscillations". In: Trends in cognitive sciences 13.6 (2009), pp. 239-245.

[37] Paul Miller and Donald B Katz. "Stochastic transitions between neural states in taste processing and decision-making". In: Journal of Neuroscience 30.7 (2010), pp. 2559-2570.

[38] Sebastian Musslick et al. "A computational model of control allocation based on the expected value of control". In: The 2nd multidisciplinary conference on reinforcement learning and decision making. 2015.

[39] Sebastian Musslick et al. "Constraints associated with cognitive control and the stability-flexibility dilemma." In: CogSci. 2018.

[40] Sebastian Musslick et al. "Stability-flexibility dilemma in cognitive control: a dynamical system perspective". In: Proceedings of the 41st Annual Meeting of the Cognitive Science Society. 2019.

[41] Zachary L Newman et al. "Determinants of synapse diversity revealed by super-resolution quantal transmission and active zone imaging". In: Nature Communications 13.1 (2022), pp. 1-18.

[42] Darren Pais et al. "A mechanism for value-sensitive decision-making". English. In: PloS one 8.9 (2013), e73216-e73216.

[43] Charles Parkhurst and Robert L Feller. "Who invented the color wheel?" In: Color Research $\mathscr{E}$ Application 7.3 (1982), pp. 217-230.

[44] Mohandas Pillai, Joshua Goglio, and Thad G. Walker. "Matrix Numerov method for solving Schrödinger's equation". English. In: American journal of physics 80.11 (2012), pp. 1017-1019.

[45] Roger Ratcliff. "A theory of memory retrieval". English. In: Psychological review 85.2 (1978), pp. 59-108.

[46] John H. Reynolds, Leonardo Chelazzi, and Robert Desimone. "Competitive Mechanisms Subserve Attention in Macaque Areas V2 and V4". English. In: The Journal of neuroscience 19.5 (1999), pp. 17361753 .

[47] Morgan Rosendahl, Anastasia Bizyaeva, and Jonathan Cohen. "A Novel Quantum Approach to the Dynamics of Decision Making". In: (2020). 
[48] Morgan Rosendahl and Jonathan Cohen. "A Discrete Formulation of Two Alternative Forced Choice Decision Dynamics Derived from a Double-Well Quantum Landscape". In: bioRxiv (2021). DOI: 10.1101/ 2021 .09. 23.461524. eprint: https: //www . biorxiv. org/content/ early/2021/09/24/2021 .09.23 . 461524 . full . pdf. URL: https : //www . biorxiv . org/content/ early/2021/09/24/2021 .09. 23. 461524.

[49] Daniel Schroeder. Notes On Quantum Mechanics. 2020. URL: https:// physics. weber.edu/schroeder/quantum/QuantumBook. pdf (visited on $05 / 26 / 2021)$.

[50] Michael N. Shadlen and William T. Newsome. "The Variable Discharge of Cortical Neurons: Implications for Connectivity, Computation, and Information Coding". English. In: The Journal of neuroscience 18.10 (1998), pp. 3870-3896.

[51] Amitai Shenhav, Matthew M Botvinick, and Jonathan D Cohen. "The expected value of control: an integrative theory of anterior cingulate cortex function". In: Neuron 79.2 (2013), pp. 217-240.

[52] Charles F Stevens. "Quantal release of neurotransmitter and long-term potentiation". In: Cell 72 (1993), pp. 55-63.

[53] Satohiro Tajima et al. "Optimal policy for multi-alternative decisions". English. In: Nature neuroscience 22.9 (2019), pp. 1503-1511.

[54] Jennifer S. Trueblood and Jerome R. Busemeyer. "A Quantum Probability Account of Order Effects in Inference". English. In: Cognitive science 35.8 (2011), pp. 1518-1552.

[55] Naoshige Uchida, Adam Kepecs, and Zachary F Mainen. "Seeing at a glance, smelling in a whiff: rapid forms of perceptual decision making". In: Nature Reviews Neuroscience 7.6 (2006), pp. 485-491.

[56] Kai Ueltzhöffer, Diana JN Armbruster-Genç, and Christian J Fiebach. "Stochastic dynamics underlying cognitive stability and flexibility". In: PLoS computational biology 11.6 (2015), e1004331.

[57] Marius Usher and James L. McClelland. "The Time Course of Perceptual Choice: The Leaky, Competing Accumulator Model". English. In: Psychological review 108.3 (2001), pp. 550-592. 
[58] Dimitri Van Der Linden, Mattie Tops, and Arnold B Bakker. "The Neuroscience of the Flow State: Involvement of the Locus Coeruleus Norepinephrine System". In: Frontiers in Psychology 12 (2021).

[59] Bram Van Vugt et al. "The threshold for conscious report: Signal loss and response bias in visual and frontal cortex". In: Science 360.6388 (2018), pp. 537-542.

[60] Rufin VanRullen. "Perceptual Cycles". English. In: Trends in cognitive sciences 20.10 (2016), pp. 723-735.

[61] Rufin VanRullen, Thomas Carlson, and Patrick Cavanagh. "The Blinking Spotlight of Attention". English. In: Proceedings of the National Academy of Sciences - PNAS 104.49 (2007), pp. 19204-19209.

[62] Rufin VanRullen et al. "Attention-Driven Discrete Sampling of Motion Perception". English. In: Proceedings of the National Academy of Sciences - PNAS 102.14 (2005), pp. 5291-5296.

[63] Corey N. White, Roger Ratcliff, and Jeffrey J. Starns. "Diffusion models of the flanker task: Discrete versus gradual attentional selection". English. In: Cognitive psychology 63.4 (2011), pp. 210-238.

[64] Robert M Yerkes, John D Dodson, et al. "The relation of strength of stimulus to rapidity of habit-formation". In: Punishment: Issues and experiments (1908), pp. 27-41. 